Supporting Information for:

\title{
Synthesis of Functionalized 1,4-Azaborinines by the Cyclization of Di-tert- butyliminoborane and Alkynes
}

\author{
Marius Schäfer ${ }^{\dagger}$ Nicholas A. Beattie, ${ }^{\S}$ K. Geetharani, ${ }^{\dagger}$ Julian Schäfer,${ }^{\$}$ William C. Ewing,${ }^{\dagger}$ \\ Mirjam Krahfuß ${ }^{\dagger}$ Christian Hörl, ${ }^{\dagger}$ Rian D. Dewhurst, ${ }^{\dagger}$ Stuart A. Macgregor, ${ }^{*} \S$ Christoph \\ Lambert, ${ }^{* \$}$ and Holger Braunschweig, ${ }^{*} \uparrow$ \\ ${ }^{\dagger}$ Institut für anorganische Chemie, Julius-Maximilians-Universität Würzburg, Am Hubland, \\ 97074 Würzburg, Germany. \\ $\S$ Institute of Chemical Sciences, Heriot-Watt University, Edinburgh, UK EH14 4AS. \\ \$ Insitute für organische Chemie, Julius-Maximilians-Universität Würzburg, Am Hubland, \\ 97074 Würzburg, Germany.
}


All manipulations were performed either under an atmosphere of dry argon or in vacuo using standard Schlenk line or glovebox techniques. $\mathrm{C}_{6} \mathrm{D}_{6}$ was dried over molecular sieves and degassed by freeze-pump-thaw cycles before use. All other solvents were distilled from appropriate drying agents. Solvents were stored under argon over activated molecular sieves. NMR spectra of isolated compounds were acquired on a Bruker Avance 500 NMR spectrometer. Chemical shifts $(\delta)$ are given in ppm. ${ }^{1} \mathrm{H}$ and ${ }^{13} \mathrm{C}\left\{{ }^{1} \mathrm{H}\right\}$ NMR spectra were referenced to external tetramethylsilane via the residual protio solvent $\left({ }^{1} \mathrm{H}\right)$ or the solvent itself $\left({ }^{13} \mathrm{C}\right) .{ }^{11} \mathrm{~B}\left\{{ }^{1} \mathrm{H}\right\}$ NMR spectra were referenced to external $\mathrm{BF}_{3} \cdot \mathrm{OEt}_{2},{ }^{31} \mathrm{P}\left\{{ }^{1} \mathrm{H}\right\} \mathrm{NMR}$ spectra to $85 \% \mathrm{H}_{3} \mathrm{PO}_{4}$. Infrared data were acquired on a JASCO FT/IR-6200type A apparatus. Microanalyses were performed on an Elementar vario MICRO cube elemental analyzer. 2ethynyl-4,4,5,5-tetramethyl-1,3,2-dioxaborolane, phenyl acetylene, 1,4-diethynyl benzene, 1,7-octadiyne and 1,3,5-triethynylbenzene were purchased from TCI Chemicals and Sigma Aldrich Chemical Co. $\quad\left[\left({ }^{i} \mathrm{Pr}_{3} \mathrm{P}\right)_{2} \mathrm{RhCl}\right]_{2}(\mathbf{1}),{ }^{1} \quad t \mathrm{BuB} \equiv \mathrm{N} t \mathrm{Bu}(\mathbf{2}),{ }^{2} \quad\left[\eta^{4}-1,2-\right.$

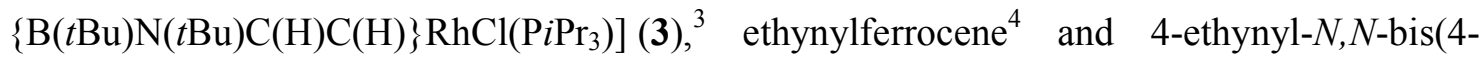
methoxyphenyl)aniline ${ }^{5}$ were synthesized according to literature procedures.

\section{Steady-state absorption spectroscopy:}

The UV/vis/NIR absorption spectra were measured in $1 \mathrm{~cm}$ quartz cuvettes from Hellma or Starna using a Jasco V-670 UV/Vis/NIR spectrometer (software SpectraManager v. 2.08.04) at rt. All solvents were spectroscopic grade and were used without further purification (Uvasol from Merck). Aggregation of the samples could be excluded by concentration independent behavior $\left(10^{-6}-10^{-5} \mathrm{M}\right)$. The pure solvent was used as reference.

\section{Emission spectroscopy:}

Steady-state fluorescence measurements were performed with an Edinburgh Instruments FLS980 spectrometer. The compounds were dissolved in spectroscopic grade solvents (Uvasol from Merck) and purged with argon gas for $30 \mathrm{~min}$ prior to each measurement. Fluorescence quantum yields were measured with an integrating sphere. Fluorescence lifetimes were determined by time-correlated single-photon counting (TCSPC) by exciting the samples with a pulsed LED at $31600 \mathrm{~cm}^{-1}(316 \mathrm{~nm})$ under magic-angle conditions and using a fast PMT detector (H10720) for fluorescence detection. Deconvolution of the data (4096 channels) was done by measuring the instrument response function with a scatterer (LUDOX). 


\section{Cyclic Voltammetry (CV):}

Cyclic voltammetry measurenments were performed with a Gamry Instruments Reference 600 Potentiostat/Galvanostat/ZRA (v. 6.2.2, Warminster, PA, USA). The cyclic voltammograms were measured under an argon atmosphere in $\mathrm{CH}_{2} \mathrm{Cl}_{2}$ with tetrabutylammonium hexafluorophosphate $(0.2 \mathrm{M})$ as conducting salt. A conventional three electrode set-up consisting of a platinum disc working electrode $(\varnothing=1 \mathrm{~mm}), \mathrm{a} \mathrm{Ag} / \mathrm{AgCl}$ 'LEAK FREE' reference electrode (Warner Instruments, Hamden, CT, USA) and a platinum wire counter electrode was used. The measurement cell was dried in an oven and flushed with argon before use. The reference electrode was referenced against the ferrocene/ferrocenium $\left(\mathrm{Fc} / \mathrm{Fc}^{+}\right)$redox couple. Chemical and electrochemical reversability of the redox processes were checked by multiple thin layer experiments and measurements at different scan rates (from $25-1000 \mathrm{mV} \mathrm{s}^{-1}$ ), respectively.

\section{Mass spectrometry:}

Mass spectra were recorded with a Bruker Daltonic microTOF focus (ESI). All mass spectrometry peaks are reported as $\mathrm{m} / \mathrm{z}$. For calculation of the respective mass values of the isotopic distribution, the software module "Bruker Daltonics IsotopePattern" from the software Compass 1.1 from Bruker Daltonics GmbH was used.

\section{$\underline{\text { Computational Details }}$}

DFT calculations were run with Gaussian 03 (Revision D.01) and Gaussian 09 (Revision D.01). $\mathrm{Rh}, \mathrm{P}$ and $\mathrm{Cl}$ centres were described with the Stuttgart RECPs and associated basis sets with addition polarization of $\mathrm{P}(\varsigma=0.387)$ and $\mathrm{Cl}(\varsigma=0.640)$. Meanwhile, 6-31 $\mathrm{g} * *$ basis sets were used for all other atoms. Initial BP86 optimizations were performed with Gaussian 03 with all stationary points being fully characterized via analytical frequency calculations (run with Gaussian 09) as either minima (all positive eigenvalues) or transition states (one negative eigenvalue). IRC calculations and subsequent geometry optimizations with Gaussian 03 were used to confirm the minima linked by each transition state. PCM corrections for the effects of benzene solvent $(\varepsilon=2.27)$ were computed using Gaussian 09 and dispersion corrections applied using Grimme's D3 parameter set using the BP86optimized geometries. 


\section{Synthesis of $\left[\eta^{4}-1,2-\left\{\mathrm{B}(t \mathrm{Bu}) \mathrm{N}(t \mathrm{Bu}) \mathrm{C}(\mathrm{H}) \mathrm{C}\left(\mathrm{C}_{6} \mathrm{H}_{5}\right)\right\} \operatorname{RhCl}\left(\mathrm{PiPr}_{3}\right)\right](3)$}

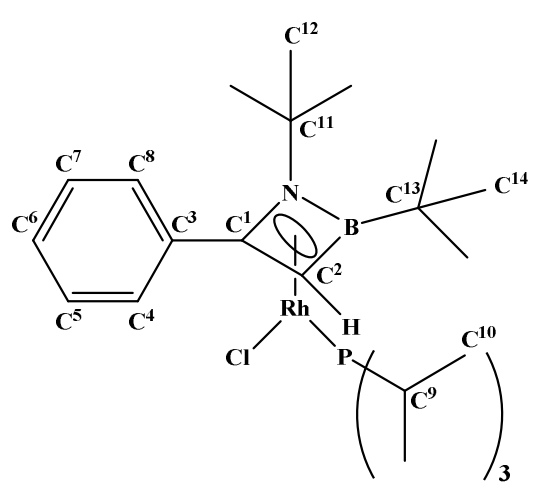

In a Schlenk tube, $\left[\left(i \mathrm{Pr}_{3} \mathrm{P}\right)_{2} \mathrm{RhCl}\right]_{2}(\mathbf{1})(693 \mathrm{mg}, 0.76 \mathrm{mmol})$, phenylacetylene (154 mg, 1.51 $\mathrm{mmol})$ and $t \mathrm{BuB} \equiv \mathrm{N} t \mathrm{Bu}(2)(500 \mathrm{mg}, 3.60 \mathrm{mmol})$ were dissolved in THF $(5 \mathrm{~mL})$ and stirred overnight at ambient temperature. After removing all volatiles in vacuo the residue was extracted with pentane at $-30{ }^{\circ} \mathrm{C}$ and dried under vacuum to yield $\mathbf{3}$ as an orange air- and moisture-sensitive powder $(0.557 \mathrm{~g}, 1.03 \mathrm{mmol}, 68 \%$ yield based on phenylacetylene). Crystals suitable for X-ray diffraction were grown by slow evaporation of a saturated pentane solution of 3 at $-30{ }^{\circ} \mathrm{C}$.

${ }^{1} \mathrm{H}$ NMR $\left(500.1 \mathrm{MHz}, \mathrm{C}_{6} \underline{D}_{6}, 296 \mathrm{~K}\right): \delta=7.86\left(\mathrm{~m}, 2 \mathrm{H}, \mathrm{C}^{5} \mathrm{H}, \mathrm{C}^{7} \mathrm{H}\right), 7.01\left(\mathrm{~m}, 3 \mathrm{H}, \mathrm{C}^{4} \mathrm{H}, \mathrm{C}^{6} \mathrm{H}\right.$, $\left.\mathrm{C}^{8} \mathrm{H}\right), 3.24$ (s, 1H, $\left.\mathrm{C}^{2} \mathrm{H}\right), 2.06\left(\mathrm{~m}, 3 \mathrm{H}, \mathrm{C}^{9} \mathrm{H}\right), 1.41\left(\mathrm{~s}, 9 \mathrm{H}, \mathrm{C}^{14} \mathrm{H}\right), 1.38\left(\mathrm{~s}, 9 \mathrm{H}, \mathrm{C}^{12} \mathrm{H}\right), 1.10$ (dd, $\left.9 \mathrm{H}, \mathrm{C}^{10} \mathrm{H},{ }^{3} J_{\mathrm{PH}}=14 \mathrm{~Hz},{ }^{3} J_{\mathrm{HH}}=7 \mathrm{~Hz}\right), 1.05 \mathrm{ppm}\left(\mathrm{dd}, 9 \mathrm{H}, \mathrm{C}^{10} \mathrm{H},{ }^{3} J_{\mathrm{PH}}=14 \mathrm{~Hz},{ }^{3} J_{\mathrm{HH}}=7 \mathrm{~Hz}\right)$.

${ }^{13} \mathrm{C}$ NMR $\left(125.8 \mathrm{MHz}, \mathrm{C}_{6} \underline{\mathrm{D}}_{6}, 296 \mathrm{~K}\right): \delta=134.5\left(\mathrm{C}^{3}\right), 131.6\left(\mathrm{C}^{5}, \mathrm{C}^{7}\right), 129.3\left(\mathrm{C}^{6}\right), 127.6\left(\mathrm{C}^{4}\right.$, $\left.\mathrm{C}^{8}\right), 105.2\left(\mathrm{dd}, \mathrm{C}^{1},{ }^{1} J_{\mathrm{RhC}}=14.0 \mathrm{~Hz},{ }^{2} J_{\mathrm{PC}}=3.0 \mathrm{~Hz}\right), 56.7\left(\mathrm{C}^{11}\right), 49.9\left(\mathrm{br}, \mathrm{C}^{2}\right), 30.3\left(\mathrm{C}^{12}\right), 29.7$ $\left(\mathrm{C}^{14}\right), 25.5\left(\mathrm{dd}, \mathrm{C}^{9},{ }^{1} J_{\mathrm{PC}}=21 \mathrm{~Hz},{ }^{2} J_{\mathrm{RhC}}=1 \mathrm{~Hz}\right), 20.6\left(\mathrm{C}^{10}\right), 20.1\left(\mathrm{C}^{10}\right), 17.1 \mathrm{ppm}\left(\mathrm{br}, \mathrm{C}^{13}\right)$.

${ }^{11} \mathrm{~B}$ NMR $\left(160.5 \mathrm{MHz}, \mathrm{C}_{6} \underline{D}_{6}, 296 \mathrm{~K}\right): \delta=25.2 \mathrm{ppm}(\mathrm{br})$.

${ }^{31} \mathrm{P}$ NMR $\left(202.5 \mathrm{MHz}, \mathrm{C}_{6} \underline{\mathrm{D}_{6}} \underline{6}, 296 \mathrm{~K}\right): \delta=60.9 \mathrm{ppm}\left(\mathrm{d},{ }^{1} J_{\mathrm{RhP}}=193.0 \mathrm{~Hz}\right)$.

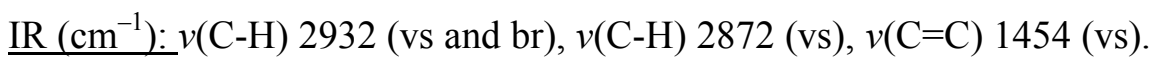

Elemental analysis calcd. [\%] for $\mathrm{C}_{25} \mathrm{H}_{45} \mathrm{BClNPRh}$ : C, 55.63; H, 8.40; N, 2.59. Found: C, $55.67 ; \mathrm{H}, 8.36 ; \mathrm{N}, 2.54$. 


\section{Synthesis of 1,4-di-tert-butyl-2-phenyl-1,4-azaborinine (4)}

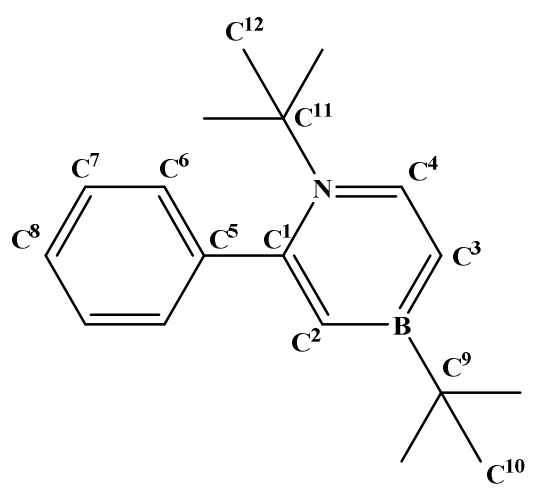

In a Schlenk tube, $\left[\eta^{4}-1,2-\{\mathrm{B}(t \mathrm{Bu}) \mathrm{N}(t \mathrm{Bu}) \mathrm{C}(\mathrm{H}) \mathrm{C}(\mathrm{H})\} \mathrm{RhCl}\left(\mathrm{P} i P r_{3}\right)\right](3)(100 \mathrm{mg}, 0.185 \mathrm{mmol})$, was dissolved in benzene ( $2 \mathrm{~mL})$ and phenylacetylene $(20 \mathrm{mg}, 0.195 \mathrm{mmol})$ was added. The reaction mixture was heated to reflux with a heat gun over $10 \mathrm{~min}$, all volatiles were removed in vacuo and the residue was purified via flash chromatography using silica gel with a mixture of pentane and ether (20:1) as eluent. The first fraction yielded $\mathbf{4}$ as a colorless solid (31 mg, $0.116 \mathrm{mmol}, 63 \%$ ). X-ray quality crystals were grown by slow evaporation of a pentane solution of 4 at $-30{ }^{\circ} \mathrm{C}$.

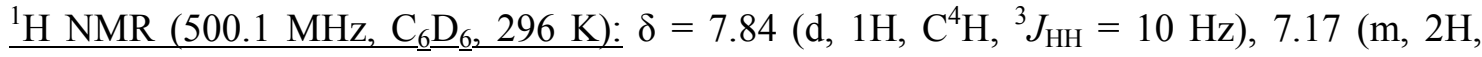
$\left.\mathrm{C}^{6} \mathrm{H}\right), 7.01\left(\mathrm{~m}, 3 \mathrm{H}, \mathrm{C}^{7} \mathrm{H}, \mathrm{C}^{8} \mathrm{H}\right), 6.74\left(\mathrm{dd}, 1 \mathrm{H}, \mathrm{C}^{3} \mathrm{H},{ }^{3} J_{\mathrm{HH}}=10 \mathrm{~Hz},{ }^{4} J_{\mathrm{HH}}=3 \mathrm{~Hz}\right), 6.62(\mathrm{~d}, 1 \mathrm{H}$, $\left.\mathrm{C}^{2} \mathrm{H},{ }^{4} J_{\mathrm{HH}}=3 \mathrm{~Hz}\right), 1.45\left(\mathrm{~s}, 9 \mathrm{H}, \mathrm{C}^{10} \mathrm{H}\right), 0.98 \mathrm{ppm}\left(\mathrm{s}, 9 \mathrm{H}, \mathrm{C}^{12} \mathrm{H}\right)$.

${ }^{13} \mathrm{C}$ NMR $\left(125.8 \mathrm{MHz}, \mathrm{C}_{6} \underline{\mathrm{D}_{6}}, 296 \mathrm{~K}\right): \delta=154.8\left(\mathrm{C}^{1}\right), 144.8\left(\mathrm{C}^{5}\right), 129.9\left(\mathrm{C}^{4}\right), 129.5\left(\mathrm{C}^{6}\right)$, $128.3\left(C^{7}\right), 127.7\left(C^{2}\right), 127.4\left(C^{8}\right), 119.1\left(b r, C^{3}\right), 62.9\left(C^{11}\right), 32.9\left(C^{12}\right), 30.3\left(C^{10}\right), 20.9$ ppm (br, $\left.\mathrm{C}^{9}\right)$.

${ }^{11} \mathrm{~B}$ NMR $\left(160.5 \mathrm{MHz}, \mathrm{C}_{6} \underline{\mathrm{D}}_{6}, 296 \mathrm{~K}\right): \delta=46.4 \mathrm{ppm}(\mathrm{br})$.

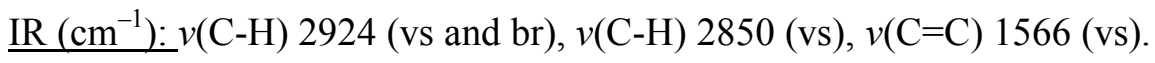

Elemental analysis calcd. [\%] for $\mathrm{C}_{18} \mathrm{H}_{26} \mathrm{BN}$ : C, 80.91; H, 9.81; N, 5.24. Found: C, 80.60; H, 9.85; N, 5.50. 


\section{Synthesis of 1,4-di-tert-butyl-2-ferrocenyl-1,4-azaborinine (6)}

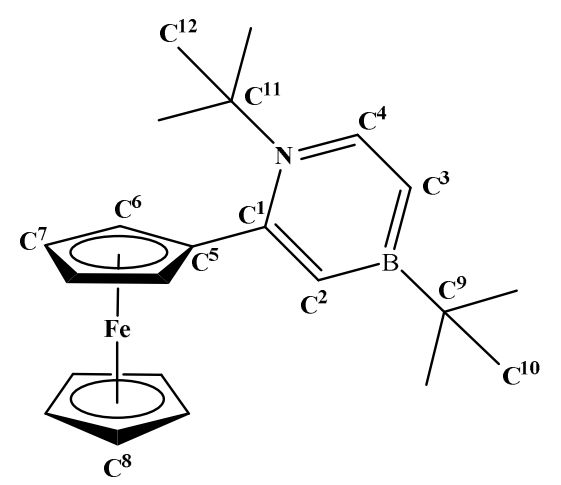

In a Schlenk tube, $\left[\left(i \mathrm{Pr}_{3} \mathrm{P}\right)_{2} \mathrm{RhCl}\right]_{2}(\mathbf{1})(575 \mathrm{mg}, 1.25 \mathrm{mmol})$, ethynylferrocene $(263 \mathrm{mg}, 1.25$ mmol) and $t \mathrm{BuB} \equiv \mathrm{N} t \mathrm{Bu}(2)(500 \mathrm{mg}, 3.60 \mathrm{mmol})$ were dissolved in THF $(5 \mathrm{~mL})$ and stirred overnight at ambient temperature. After removing all volatiles in vacuo the residue was dissolved in benzene $(4 \mathrm{~mL})$, put under an atmosphere of acetylene and the reaction mixture was heated to reflux with a heat gun for $10 \mathrm{~min}$. After removing all volatiles in vacuo, the residue was purified by via flash chromatography using silica gel with a mixture of pentane and ether (20:1) as eluent. The first fraction yielded 6 as a red solid (256 mg, $0.68 \mathrm{mmol}$, $55 \%$ ). X-ray quality crystals were grown by slow evaporation of a pentane solution of 6 at $30{ }^{\circ} \mathrm{C}$.

${ }^{1} \mathrm{H}$ NMR $\left(500.1 \mathrm{MHz}, \mathrm{C}_{6} \underline{D}_{6}, 296 \mathrm{~K}\right): \delta=7.75\left(\mathrm{~d}, 1 \mathrm{H}, \mathrm{C}^{2} \mathrm{H},{ }^{4} J_{\mathrm{HH}}=3 \mathrm{~Hz}\right), 7.74\left(\mathrm{~d}, 1 \mathrm{H}, \mathrm{C}^{4} \mathrm{H}\right.$, $\left.{ }^{3} J_{\mathrm{HH}}=10 \mathrm{~Hz}\right), 6.63\left(\mathrm{dd}, 1 \mathrm{H}, \mathrm{C}^{3} \mathrm{H},{ }^{3} J_{\mathrm{HH}}=10 \mathrm{~Hz},{ }^{4} J_{\mathrm{HH}}=3 \mathrm{~Hz}\right), 4.38\left(\mathrm{vt}, 2 \mathrm{H}, \mathrm{C}^{6} \mathrm{H}\right), 4.11(\mathrm{~s}, 5 \mathrm{H}$, $\left.\mathrm{C}^{8} \mathrm{H}\right), 3.94$ (vt, 2H, $\left.\mathrm{C}^{7} \mathrm{H}\right), 1.55$ (s, 9H, $\left.\mathrm{C}^{10} \mathrm{H}\right), 1.01 \mathrm{ppm}\left(\mathrm{s}, 9 \mathrm{H}, \mathrm{C}^{12} \mathrm{H}\right)$.

${ }^{13} \mathrm{C}$ NMR $\left(125.8 \mathrm{MHz}, \mathrm{C}_{6} \underline{\mathrm{D}}_{6}, 296 \mathrm{~K}\right): \delta=150.7\left(\mathrm{C}^{1}\right), 143.6\left(\mathrm{C}^{4}\right), 131.3\left(\mathrm{br}, \mathrm{C}^{2}\right), 118.3$ (br, $\left.\mathrm{C}^{3}\right), 96.7\left(\mathrm{C}^{5}\right), 72.4\left(\mathrm{C}^{6}\right), 70.7\left(\mathrm{C}^{8}\right), 66.9\left(\mathrm{C}^{7}\right), 62.6\left(\mathrm{C}^{9}\right), 32.8\left(\mathrm{C}^{12}\right), 30.7\left(\mathrm{C}^{10}\right), 21.3 \mathrm{ppm}(\mathrm{br}$, $\left.\mathrm{C}^{9}\right)$.

${ }^{11} \mathrm{~B}$ NMR $\left(160.5 \mathrm{MHz}, \mathrm{C}_{6} \underline{\mathrm{D}}_{6}, 296 \mathrm{~K}\right): \delta=46.4 \mathrm{ppm}(\mathrm{br})$.

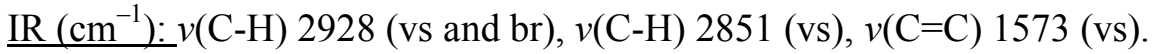

Elemental analysis calcd. [\%] for $\mathrm{C}_{20} \mathrm{H}_{30} \mathrm{BNFe}$ C, 70.44; $\mathrm{H}, 8.06$; N, 3.73. Found: C, 70.53; H, 8.06; N, 3.89 . 


\section{Synthesis of 1,4-di-tert-butyl-2-pinacolboryl-1,4-azaborinine (7)}

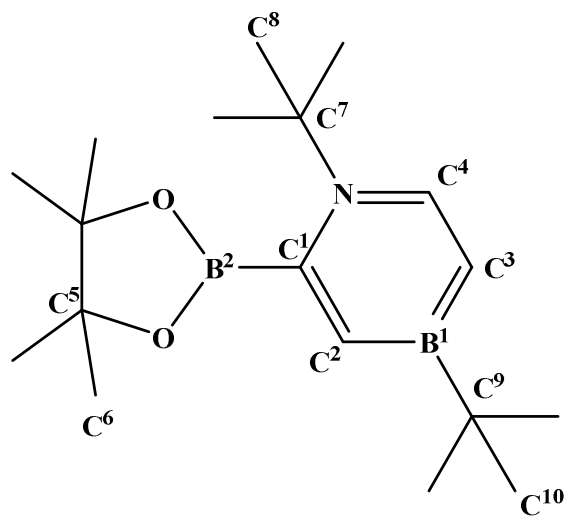

In a Schlenk tube, $\left[\eta^{4}-1,2-\{\mathrm{B}(t \mathrm{Bu}) \mathrm{N}(t \mathrm{Bu}) \mathrm{C}(\mathrm{H}) \mathrm{C}(\mathrm{H})\} \mathrm{RhCl}\left(\mathrm{P} i P r_{3}\right)\right](1.59 \mathrm{~g}, 3.50 \mathrm{mmol})$ and 2ethynyl-4,4,5,5-tetramethyl-1,3,2-dioxaborolane (0.532 g, $3.50 \mathrm{mmol})$ were dissolved in benzene $(10 \mathrm{~mL})$ and heated to reflux for $10 \mathrm{~min}$. After removing all volatiles in vacuo the residue was dissolved in benzene $(4 \mathrm{~mL})$, placed under an atmosphere of acetylene and the reaction mixture was heated to reflux with a heat gun for $10 \mathrm{~min}$. After removing all volatiles in vacuo, the residue was purified by via flash chromatography using silica gel with a mixture of pentane and ether (10:1) as eluent. The first fraction yielded 7 as a colorless solid (404 mg, $1.37 \mathrm{mmol}, 39 \%)$. X-ray quality crystals were grown by slow evaporation of a pentane solution of 7 at $-30{ }^{\circ} \mathrm{C}$.

$\underline{{ }^{1} \mathrm{H} N M R}\left(500.1 \mathrm{MHz}, \mathrm{C}_{6} \underline{D}_{6}, 296 \mathrm{~K}\right): \delta=7.81\left(\mathrm{~d}, 1 \mathrm{H}, \mathrm{C}^{4} \mathrm{H},{ }^{3} \mathrm{~J}_{\mathrm{HH}}=10 \mathrm{~Hz}\right), 7.33\left(\mathrm{~d}, 1 \mathrm{H}, \mathrm{C}^{2} \mathrm{H}\right.$, $\left.{ }^{4} J_{\mathrm{HH}}=3 \mathrm{~Hz}\right), 6.90\left(\mathrm{dd}, 1 \mathrm{H}, \mathrm{C}^{3} \mathrm{H},{ }^{3} J_{\mathrm{HH}}=10 \mathrm{~Hz},{ }^{4} J_{\mathrm{HH}}=3 \mathrm{~Hz}\right), 1.42\left(\mathrm{~s}, 9 \mathrm{H}, \mathrm{C}^{10} \mathrm{H}\right) 1.32(\mathrm{~s}, 9 \mathrm{H}$, $\left.\mathrm{C}^{8} \mathrm{H}\right), 1.13 \mathrm{ppm}\left(\mathrm{s}, 12 \mathrm{H}, \mathrm{C}^{6} \mathrm{H}\right)$.

${ }^{13} \mathrm{C}$ NMR $\left(125.8 \mathrm{MHz}, \mathrm{C}_{6} \underline{\mathrm{D}}_{6}, 296 \mathrm{~K}\right): \delta=141.3\left(\mathrm{C}^{4}\right), 129.3\left(\mathrm{C}^{2}\right), 128.4\left(\mathrm{C}^{1}\right), 119.6\left(\mathrm{C}^{3}\right), 84.0$ $\left(C^{5}\right), 60.9\left(C^{7}\right), 31.2\left(C^{8}\right), 30.4\left(C^{10}\right), 24.6\left(C^{6}\right), 20.2$ ppm (bs, $\left.C^{9}\right)$.

${ }^{11} \mathrm{~B}$ NMR $\left(160.5 \mathrm{MHz}, \mathrm{C}_{6} \underline{\mathrm{D}}_{6}, 296 \mathrm{~K}\right): \delta=46.7\left(\mathrm{bs}, \mathrm{B}^{1}\right), 30.1 \mathrm{ppm}\left(\mathrm{bs}, \mathrm{B}^{2}\right)$.

$\underline{\mathrm{IR}\left(\mathrm{cm}^{-1}\right):} v(\mathrm{C}-\mathrm{H}) 2924$ (vs and br), v(C-H) 2844 (vs), v(C=C) 1587 (vs).

EI-MS: calc. for $\mathrm{C}_{18} \mathrm{H}_{33} \mathrm{~B}_{2} \mathrm{NO}_{2}: 317.27 \mathrm{~m} / \mathrm{z}$, found: $317.3 \mathrm{~m} / \mathrm{z}(\mathrm{M}+), 302.3 \mathrm{~m} / \mathrm{z}(\mathrm{M}+-\mathrm{CH} 3)$, $260.2 \mathrm{~m} / \mathrm{z}(\mathrm{M}+-\mathrm{tBu})$. 
Synthesis of 4-(1,4-di-tert-1,4-azaborinin-2-yl)- $N, N$-bis(4-methoxyphenyl)aniline (8)

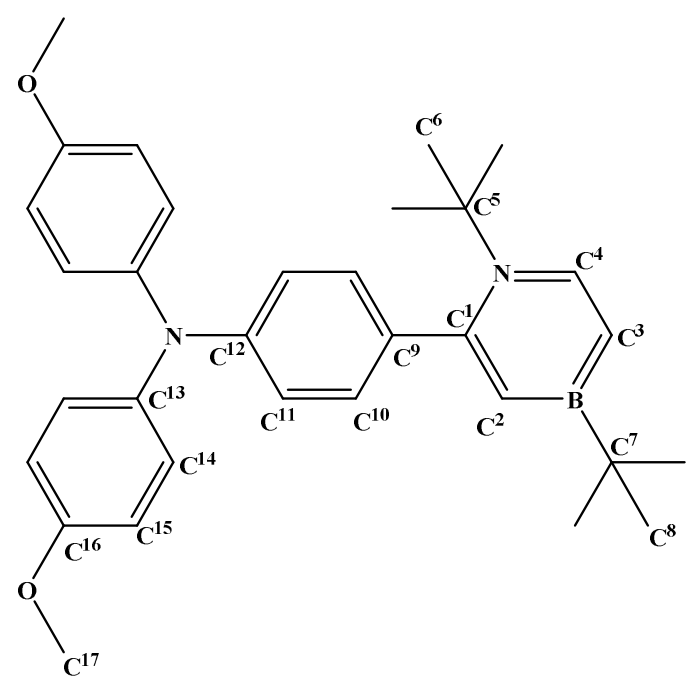

In a Schlenk tube, $\left[\eta^{4}-1,2-\{\mathrm{B}(t \mathrm{Bu}) \mathrm{N}(t \mathrm{Bu}) \mathrm{C}(\mathrm{H}) \mathrm{C}(\mathrm{H})\} \mathrm{RhCl}\left(\mathrm{P} i \operatorname{Pr}_{3}\right)\right](578 \mathrm{mg}, 1.28 \mathrm{mmol})$, was dissolved in benzene $(20 \mathrm{~mL})$ and 4-ethynyl- $N, N$-bis(4-methoxyphenyl)aniline was added (420 mg, $1.28 \mathrm{mmol})$. The reaction mixture was heated to reflux with a heatgun over $15 \mathrm{~min}$, all volatiles were removed in vacuo and the residue was purified via flash chromatography using silica gel with a mixture of petroleum ether and dichloromethane (1:1) as eluent. The first fraction yielded $\mathbf{8}$ as a colorless pale yellow solid (110 mg, $0.23 \mathrm{mmol}, 18 \%)$. X-ray quality crystals were grown by slow evaporation of a petroleum ether solution of $\mathbf{8}$.

${ }^{1} \mathrm{H}$ NMR $\left(500.1 \mathrm{MHz}, \mathrm{C}_{6} \underline{D}_{6}, 296 \mathrm{~K}\right): \delta=7.88\left(\mathrm{~m}, 1 \mathrm{H}, \mathrm{C}^{4} \mathrm{H}\right), 7.10\left(\mathrm{~m}, 6 \mathrm{H}, \mathrm{C}^{10} \mathrm{H} \& \mathrm{C}^{15} \mathrm{H}\right.$ overlapped), 6.99 (m, 2H, $\left.\mathrm{C}^{11} \mathrm{H}\right), 6.73$ (m, 6H, $\mathrm{C}^{2} \mathrm{H} \& \mathrm{C}^{3} \mathrm{H} \& \mathrm{C}^{14} \mathrm{H}$ overlapped), 3.30 (s, 6H, $\left.\mathrm{C}^{17} \mathrm{H}\right), 1.46\left(\mathrm{~s}, 9 \mathrm{H}, \mathrm{C}^{8} \mathrm{H}\right), 1.12 \mathrm{ppm}\left(\mathrm{s}, 9 \mathrm{H}, \mathrm{C}^{6} \mathrm{H}\right)$.

${ }^{13} \mathrm{C} \mathrm{NMR}\left(125.8 \mathrm{MHz}, \mathrm{C}_{6} \underline{D_{6}}, 296 \mathrm{~K}\right): \delta=156.7\left(\mathrm{~s}, \mathrm{C}^{16}\right), 155.1\left(\mathrm{~s}, \mathrm{C}^{1}\right), 148.5\left(\mathrm{~s}, \mathrm{C}^{9}\right), 142.7(\mathrm{~s}$, $\left.\mathrm{C}^{4}\right), 141.2\left(\mathrm{~s}, \mathrm{C}^{13}\right), 136.8\left(\mathrm{~s}, \mathrm{C}^{12}\right), 130.2\left(\mathrm{~s}, \mathrm{C}^{10} \& \mathrm{C}^{15}\right), 128.4\left(\mathrm{C}^{2}\right) 127.2\left(\mathrm{~s}, \mathrm{C}^{11}\right), 119.4(\mathrm{~s}$, $\left.\mathrm{C}^{14}\right), 115.3\left(\mathrm{~s}, \mathrm{C}^{3}\right), 62.9\left(\mathrm{~s}, \mathrm{C}^{5}\right), 55.0\left(\mathrm{~s}, \mathrm{C}^{17}\right), 33.0\left(\mathrm{~s}, \mathrm{C}^{6}\right), 30.1\left(\mathrm{~s}, \mathrm{C}^{8}\right), 21.0 \mathrm{ppm}\left(\mathrm{bs}, \mathrm{C}^{7}\right)$.

${ }^{11} \mathrm{~B}$ NMR $\left(160.5 \mathrm{MHz}, \mathrm{C}_{6} \underline{\mathrm{D}}_{6}, 296 \mathrm{~K}\right): \delta=47.5 \mathrm{ppm}(\mathrm{bs})$.

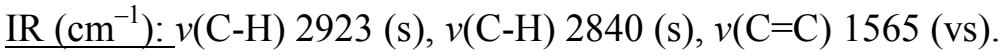

ESI-MS (M + H', high resolution): calcd.: $494.31047 \mathrm{~m} / \mathrm{z}$; found: $494.30908 \mathrm{~m} / \mathrm{z}$;

$\Delta=2.83 \mathrm{ppm}$ 


\section{Synthesis of 1,4-bis $\left[\eta^{4}-1,2-\left\{\mathrm{B}(t \mathrm{Bu}) \mathrm{N}(t \mathrm{Bu}) \mathrm{C}(\mathrm{H}) \mathrm{CRhCl}\left(\mathrm{PiPr}_{3}\right)\right]\right.$-benzene (9)}

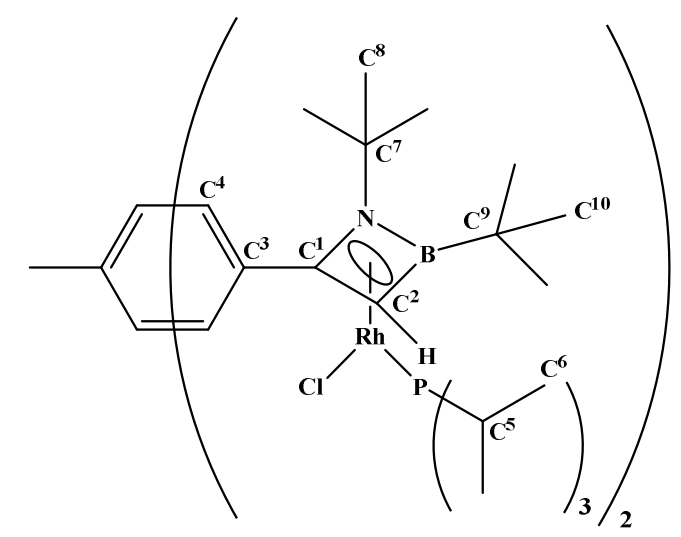

In a Schlenk tube, $\left[\left(i \mathrm{Pr}_{3} \mathrm{P}\right)_{2} \mathrm{RhCl}\right]_{2}(\mathbf{1})(154 \mathrm{mg}, 0.168 \mathrm{mmol}), 1,4$-diethynylbenzene $(21.7 \mathrm{mg}$, $0.168 \mathrm{mmol})$ and $t \mathrm{BuB} \equiv \mathrm{N} t \mathrm{Bu}(2)(200 \mathrm{mg}, 1.44 \mathrm{mmol})$ were dissolved in THF (4 mL) and stirred overnight at ambient temperature. After removing all volatiles in vacuo the residue was extracted with pentane at $-30{ }^{\circ} \mathrm{C}$ and dried under vacuum to yield $\mathbf{9}$ as a red air- and moisture-sensitive powder (39 mg, $0.04 \mathrm{mmol}, 24 \%$ yield based on 1,4-diethynyl benzene). $\mathrm{X}$-ray quality crystals of 9 were obtained by storing a saturated pentane solution at $-30{ }^{\circ} \mathrm{C}$.

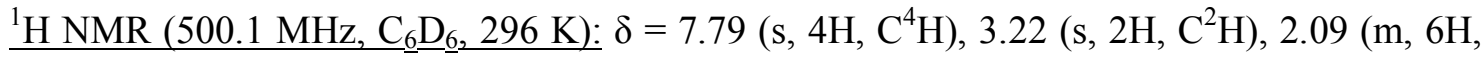
$\left.\mathrm{C}^{5} \mathrm{H}\right), 1.42$ (s, 18H, $\left.\mathrm{C}^{10} \mathrm{H}\right), 1.37$ (s, 18H, $\left.\mathrm{C}^{8} \mathrm{H}\right), 1.09 \mathrm{ppm}\left(\mathrm{m}, 36 \mathrm{H}, \mathrm{C}^{6} \mathrm{H}\right)$.

${ }^{13} \mathrm{C}$ NMR $\left(125.8 \mathrm{MHz}, \mathrm{C}_{6} \underline{\underline{\mathrm{D}}} \underline{6}, 296 \mathrm{~K}\right): \delta=130.7\left(\mathrm{C}^{4}\right), 128.6\left(\mathrm{C}^{3}\right), 104.2\left(\mathrm{C}^{1}\right), 56.7\left(\mathrm{C}^{7}\right), 49.6$ (br, $\left.\mathrm{C}^{2}\right), 56.7\left(\mathrm{C}^{11}\right), 30.3\left(\mathrm{C}^{8}\right), 29.6\left(\mathrm{C}^{10}\right), 25.5\left(\mathrm{~d}, \mathrm{C}^{5}, J_{\mathrm{PC}}=21.1 \mathrm{~Hz}\right), 20.7\left(\mathrm{C}^{6}\right), 20.1\left(\mathrm{C}^{6}\right)$, $17.1 \mathrm{ppm}\left(\mathrm{br}, \mathrm{C}^{13}\right)$.

${ }^{11} \mathrm{~B}$ NMR $\left(160.5 \mathrm{MHz}, \mathrm{C}_{6} \underline{D}_{6}, 296 \mathrm{~K}\right): \delta=26.3 \mathrm{ppm}(\mathrm{br})$.

${ }^{31} \mathrm{P}$ NMR $\left(202.5 \mathrm{MHz}, \mathrm{C}_{6} \underline{\mathrm{D}}_{6}, 296 \mathrm{~K}\right): \delta=61.1 \mathrm{ppm}\left(\mathrm{d},{ }^{1} J_{\mathrm{RhP}}=193.0 \mathrm{~Hz}\right)$.

$\underline{\mathrm{IR}\left(\mathrm{cm}^{-1}\right):} v(\mathrm{C}-\mathrm{H}) 2958$ (vs and br), $v(\mathrm{C}-\mathrm{H}) 2933$ (vs and br), $v(\mathrm{C}=\mathrm{C}) 1465(\mathrm{~m})$.

Elemental analysis calcd. [\%] for $\mathrm{C}_{44} \mathrm{H}_{84} \mathrm{~B}_{2} \mathrm{Cl}_{2} \mathrm{~N}_{2} \mathrm{P}_{2} \mathrm{Rh}_{2}$ : C, 52.77; H, 8.45; N, 2.80. Found: C, $54.78 ; \mathrm{H}, 8.43 ; \mathrm{N}, 2.44$. 


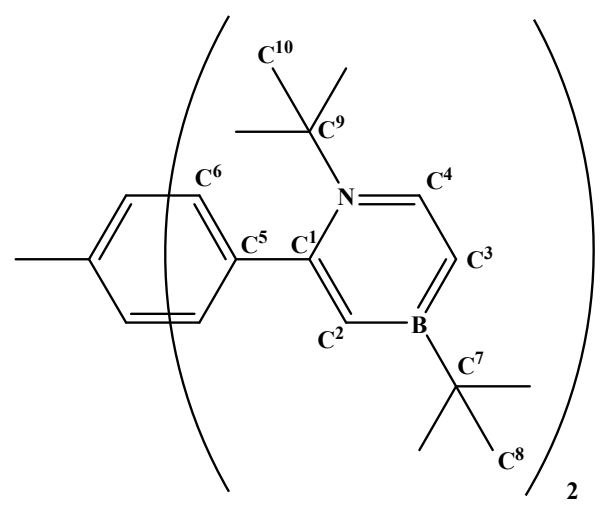

In a Schlenk tube, $\left[\left(i \mathrm{Pr}_{3} \mathrm{P}\right)_{2} \mathrm{RhCl}\right]_{2}(\mathbf{1})(235 \mathrm{mg}, 0.26 \mathrm{mmol}), 1$,4-diethynylbenzene (263 mg, $1.25 \mathrm{mmol})$ and $t \mathrm{BuB} \equiv \mathrm{N} t \mathrm{Bu}(2)(500 \mathrm{mg}, 3.60 \mathrm{mmol})$ were dissolved in THF $(5 \mathrm{~mL})$ and stirred overnight at ambient temperature. After removing all volatiles in vacuo the residue was dissolved in benzene $(4 \mathrm{~mL})$, the argon atmosphere was replaced by acetylene and the reaction mixture was heated to reflux with a heat gun for $10 \mathrm{~min}$. All volatiles were removed in vacuo and the black residue was purified by via flash chromatography using silica gel with a mixture of pentane and dichloromethane (6:1) as eluent. The first fraction yielded $\mathbf{1 0}$ as a colorless solid (73 mg, $0.22 \mathrm{mmol}, 43 \%$ ). X-ray quality crystals were grown by slow evaporation of a benzene solution of $\mathbf{1 0}$.

$\underline{{ }^{1} \mathrm{H} N M R}\left(500.1 \mathrm{MHz}, \mathrm{C}_{6} \underline{\mathrm{D}_{6}}, 296 \mathrm{~K}\right): \delta=7.84\left(\mathrm{~d}, 2 \mathrm{H}, \mathrm{C}^{4} \mathrm{H},{ }^{3} J_{\mathrm{HH}}=10 \mathrm{~Hz}\right), 7.08\left(\mathrm{~m}, 4 \mathrm{H}, \mathrm{C}^{6} \mathrm{H}\right)$, $6.76\left(\mathrm{dd}, 1 \mathrm{H}, \mathrm{C}^{3} \mathrm{H},{ }^{3} J_{\mathrm{HH}}=10 \mathrm{~Hz},{ }^{4} J_{\mathrm{HH}}=3 \mathrm{~Hz}\right), 6.73\left(\mathrm{dd}, 1 \mathrm{H}, \mathrm{C}^{3} \mathrm{H},{ }^{3} J_{\mathrm{HH}}=10 \mathrm{~Hz},{ }^{4} J_{\mathrm{HH}}=3\right.$ $\mathrm{Hz}), 6.67\left(\mathrm{~d}, 1 \mathrm{H}, \mathrm{C}^{2} \mathrm{H},{ }^{4} J_{\mathrm{HH}}=3 \mathrm{~Hz}\right), 6.61\left(\mathrm{~d}, 1 \mathrm{H}, \mathrm{C}^{2} \mathrm{H},{ }^{4} J_{\mathrm{HH}}=3 \mathrm{~Hz}\right), 1.47\left(\mathrm{~s}, 9 \mathrm{H}, \mathrm{C}^{8} \mathrm{H}\right), 1.44$ (s, 9H, C $\left.{ }^{8} \mathrm{H}\right), 1.04$ (s, 9H, C $\left.{ }^{10} \mathrm{H}\right), 0.99$ ppm (s, 9H, $\left.\mathrm{C}^{10} \mathrm{H}\right)$.

${ }^{13} \mathrm{C}$ NMR $\left(125.8 \mathrm{MHz}, \mathrm{C}_{6} \underline{\mathrm{D}}_{6}, 296 \mathrm{~K}\right): \delta=154.3\left(\mathrm{C}^{1}\right), 144.0\left(\mathrm{C}^{5}\right), 143.9\left(\mathrm{C}^{5}\right), 142.9\left(\mathrm{C}^{4}\right)$, $142.6\left(C^{4}\right), 128.7\left(C^{6}\right), 128.3\left(C^{2}\right), 119.2\left(C^{3}\right), 63.2\left(C^{9}\right), 62.9\left(C^{9}\right), 33.8\left(C^{8}\right), 32.9\left(C^{8}\right), 30.3$ $\left(\mathrm{C}^{10}\right), 21.0 \mathrm{ppm}\left(\mathrm{br}, \mathrm{C}^{7}\right)$.

${ }^{11} \mathrm{~B}$ NMR (160.5 MHz, $\left.\mathrm{C}_{6} \underline{\mathrm{D}}_{6}, 296 \mathrm{~K}\right): \delta=46.5 \mathrm{ppm}(\mathrm{br})$.

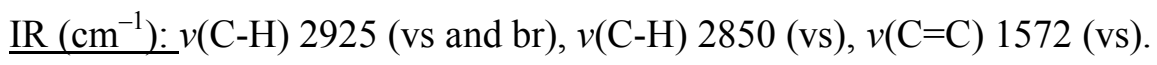

Elemental analysis calcd. [\%] for $\mathrm{C}_{30} \mathrm{H}_{46} \mathrm{~B}_{2} \mathrm{~N}_{2}$ : C, 78.96; H, 10.16; N, 6.14. Found: C, 75.71; H, 9.92; N, 6.34 . 


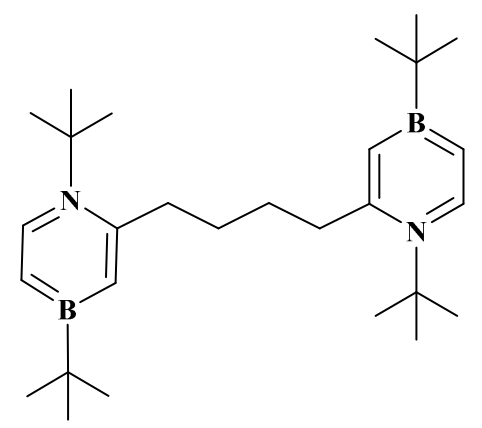

In a Schlenk tube, $\left[\left(i \operatorname{Pr}_{3} \mathrm{P}\right)_{2} \mathrm{RhCl}\right]_{2}(\mathbf{1})(400 \mathrm{mg}, 0.44 \mathrm{mmol}), 1,7$-octadiyne $(23 \mathrm{mg}, 0.22$ $\mathrm{mmol})$ and $t \mathrm{BuB} \equiv \mathrm{N} t \mathrm{Bu}(2)(50 \mathrm{mg}, 0.36 \mathrm{mmol})$ were dissolved in THF (5 mL) and stirred overnight at ambient temperature. After removing all volatiles in vacuo the residue was dissolved in benzene $(4 \mathrm{~mL})$, the argon atmosphere was replaced by acetylene and the reaction mixture was heated to reflux with a heat gun for $10 \mathrm{~min}$. All volatiles were removed in vacuo and the black residue was purified by via flash chromatography using silica gel with a mixture of pentane and dichloromethane (6:1) as eluent. The first fraction yielded $\mathbf{1 1}$ as a colorless solid (45 mg, $0.09 \mathrm{mmol}, 41 \%$ based on 1,7-octadiyne). X-ray quality crystals were grown by slow evaporation of a hexane solution of $\mathbf{1 1}$.

${ }^{1} \mathrm{H}$ NMR $\left(500.1 \mathrm{MHz}, \mathrm{C}_{6} \underline{D}_{6}, 296 \mathrm{~K}\right): \delta=7.56\left(\mathrm{~d}, 2 \mathrm{H},{ }^{3} J_{\mathrm{HH}}=10 \mathrm{~Hz}\right), 6.55\left(\mathrm{dd}, 2 \mathrm{H},{ }^{3} J_{\mathrm{HH}}=10\right.$ $\left.\mathrm{Hz},{ }^{4} J_{\mathrm{HH}}=3 \mathrm{~Hz}\right), 6.14\left(\mathrm{~d}, 2 \mathrm{H},{ }^{4} J_{\mathrm{HH}}=3 \mathrm{~Hz}\right), 2.61\left(\mathrm{t}, 4 \mathrm{H},{ }^{3} J_{\mathrm{HH}}=8 \mathrm{~Hz}\right), 1.80-1.66(\mathrm{~m}, 4 \mathrm{H}), 1.46$ (s, 18H), $0.99 \mathrm{ppm}(\mathrm{s}, 18 \mathrm{H})$.

$\underline{{ }^{13} \mathrm{C} N M R}\left(125.8 \mathrm{MHz}, \mathrm{C}_{6} \underline{\mathrm{D}}_{6}, 296 \mathrm{~K}\right): \delta=161.3,142.2,128.1,118.2$ (br), 61.7, 41.5, 33.2, 30.3, 28.6, 20.8 ppm (br).

${ }^{11} \mathrm{~B}$ NMR $\left(160.5 \mathrm{MHz}, \mathrm{C}_{6} \underline{\mathrm{D}}_{6}, 296 \mathrm{~K}\right): \delta=46.2 \mathrm{ppm}(\mathrm{br})$.

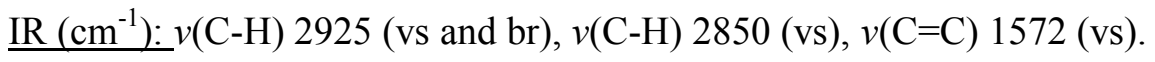

Elemental analysis calcd. [\%] for $\mathrm{C}_{28} \mathrm{H}_{50} \mathrm{~B}_{2} \mathrm{~N}_{2}$ : C, 77.07; H, 11.55; N, 6.42. Found: C, 76.98; H, 11.50; N, 6.34 .

EI-MS: m/z $436\left(\mathrm{M}^{+}\right), 379\left(\mathrm{M}^{+}{ }^{-}{ }^{\mathrm{Bu}}\right)$. 


\section{Synthesis of 1,3,5-tris(1,4-di-tert-butyl-1,4-azaborinin-2-yl)benzene (12)}

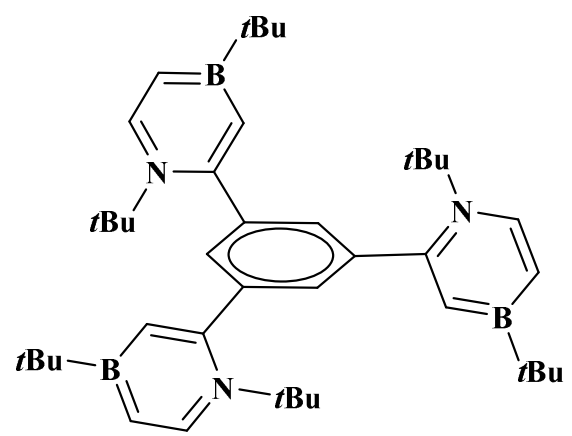

In a Schlenk tube, $\left[\left(i \mathrm{Pr}_{3} \mathrm{P}\right)_{2} \mathrm{RhCl}\right]_{2}(\mathbf{1})(270 \mathrm{mg}, 0.29 \mathrm{mmol}), 1,3,5$-triethynyl benzene $(15 \mathrm{mg}$, $0.1 \mathrm{mmol})$ and $t \mathrm{BuB} \equiv \mathrm{N} t \mathrm{Bu}(2)(25 \mathrm{mg}, 0.18 \mathrm{mmol})$ were dissolved in THF $(5 \mathrm{~mL})$ and stirred overnight at ambient temperature. After removing all volatiles in vacuo the residue was dissolved in benzene $(4 \mathrm{~mL})$, the argon atmosphere was replaced by acetylene and the reaction mixture was heated to reflux with a heat gun for $10 \mathrm{~min}$. All volatiles were removed in vacuo and the black residue was purified by via flash chromatography using silica gel with a mixture of pentane and dichloromethane $(6: 1)$ as eluent. The first fraction yielded $\mathbf{1 2}$ as a colorless solid (32 mg, $0.05 \mathrm{mmol}, 50 \%$ based on 1,3,5-triethynylbenzene). X-ray quality crystals were grown by slow evaporation of a hexane solution of $\mathbf{1 2}$.

${ }^{1} \mathrm{H}$ NMR $\left(500.1 \mathrm{MHz}, \mathrm{THF}-{ }_{2}, \underline{8}, 296 \mathrm{~K}\right): \delta=8.17-8.11(\mathrm{~m}, 3 \mathrm{H}), 7.43\left(\mathrm{t}, 1 \mathrm{H},{ }^{4} J_{\mathrm{HH}}=2 \mathrm{~Hz}\right), 7.29$ $\left(\mathrm{d}, 2 \mathrm{H},{ }^{4} J_{\mathrm{HH}}=2 \mathrm{~Hz}\right), 6.37-6.32(\mathrm{~m}, 3 \mathrm{H}), 6.23-6.15(\mathrm{~m}, 3 \mathrm{H}), 1.54(\mathrm{~s}, 18 \mathrm{H}), 1.45(\mathrm{~s}, 9 \mathrm{H}), 1.00$ (s, 9H), 0.99 ppm (s, 18H).

${ }^{13} \mathrm{C}$ NMR $\left(125.8 \mathrm{MHz}, \mathrm{THF}-{ }_{d}, \underline{8}, 296 \mathrm{~K}\right): \delta=154.5,154.4,144.5,143.5,143.4,143.2,129.6$, 128.1 (br), 127.5, 118.8 (br), 64.3, 64.1, 33.4, 33.0, 29.9, 20.8 ppm (br).

${ }^{11} \mathrm{~B}$ NMR $\left(160.5 \mathrm{MHz}, \mathrm{THF}-{ }_{\underline{8}}, 296 \mathrm{~K}\right): \delta=47.5 \mathrm{ppm}(\mathrm{br})$.

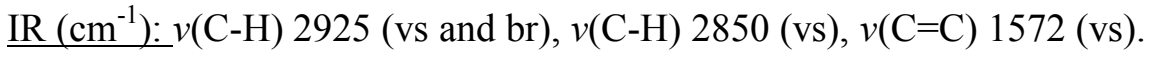

Elemental analysis calcd. [\%] for $\mathrm{C}_{42} \mathrm{H}_{66} \mathrm{~B}_{3} \mathrm{~N}_{3}$ : C, 78.16; H, 10.31; N, 6.51. Found: C, 78.04; $\mathrm{H}, 10.25 ; \mathrm{N}, 6.44$.

EI-MS: m/z $645\left(\mathrm{M}^{+}\right), 589\left(\mathrm{M}^{+}{ }^{-} \mathrm{Bu}\right)$. 


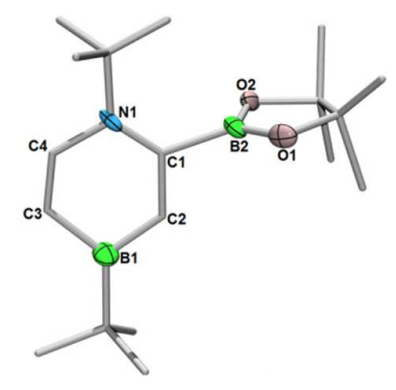

Figure S1. Molecular structure of 1,4-di-tert-butyl-2-pinacoboryl-1,4-azaborinine (7). The four crystallographically independent molecules in the asymmetric unit of 7 have nearly identical geometries, only one of which is displayed. Ellipsoids are set at 50\% probability; hydrogen atoms and some ellipsoids are omitted for clarity

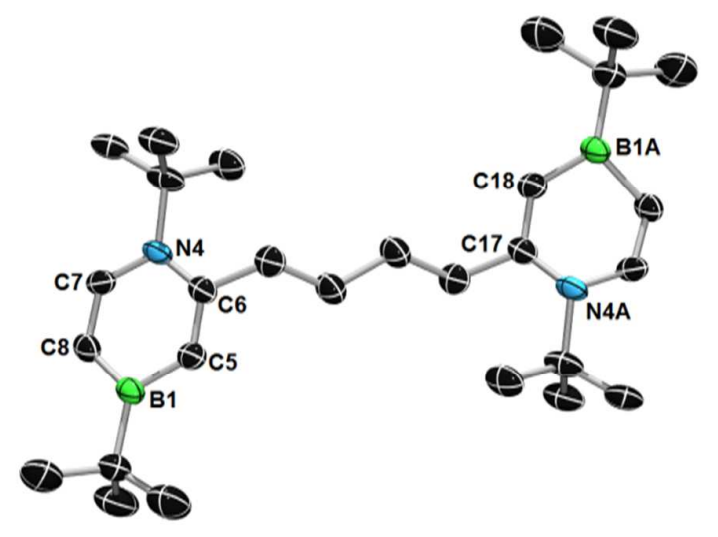

Figure S2. Molecular structure of 1,7-bis(1,4-di-tert-butyl-1,4-azaborinin-2-yl)butane (11). 

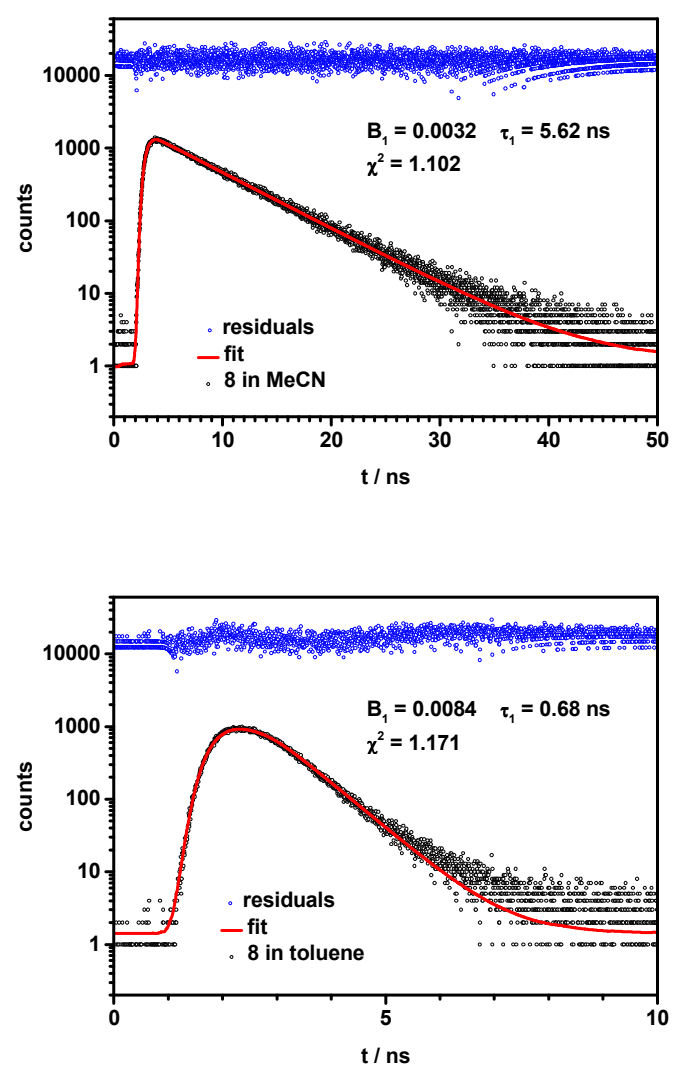

Figure S3. Fluorescence decay spectra of $\mathbf{8}$.

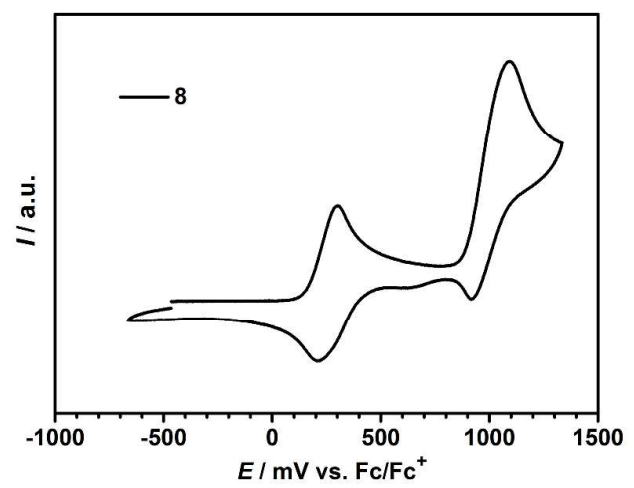

Figure S4. Cyclic voltammogram of 8 in $\mathrm{CH}_{2} \mathrm{Cl}_{2}(\mathrm{TBAH})$ at a scan rate of $2500 \mathrm{mV} \mathrm{s}^{-1}$. 


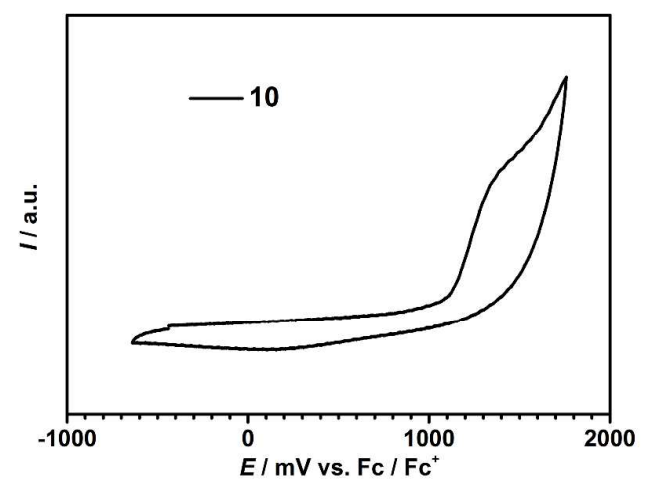

Figure S5. Cyclic voltammogram of $\mathbf{1 0}$ in $\mathrm{CH}_{2} \mathrm{Cl}_{2}$ (TBAH) at a scan rate of $2500 \mathrm{mV} \mathrm{s}^{-1}$. The cyclic voltammogram of $\mathbf{1 0}$ shows a strongly irreversible oxidation at ca. $1350 \mathrm{mV}$.

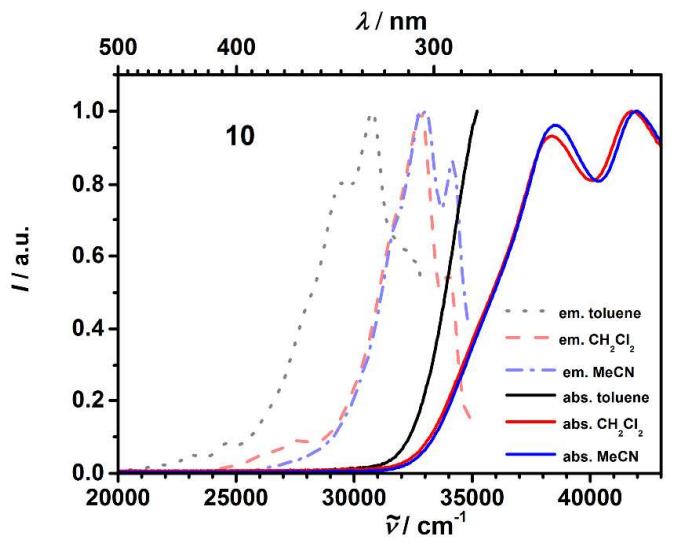

Figure S6. Normalized absorption (solid lines) and emission spectra (dashed lines) of $\mathbf{1 0}$ in toluene (black), $\mathrm{CH}_{2} \mathrm{Cl}_{2}$ (red) and $\mathrm{MeCN}$ (blue).

Table S1. Optical steady state and emission decay data of $\mathbf{1 0}$ in $\mathrm{MeCN}, \mathrm{CH}_{2} \mathrm{Cl}_{2}$ and toluene.

\begin{tabular}{|c|c|c|c|c|c|c|}
\hline & solvent & $\begin{array}{c}\tilde{v}_{\mathrm{abs}} \\
/ \mathrm{cm}^{-1}(\mathrm{~nm})^{\mathrm{a}}\end{array}$ & $\begin{array}{c}\tilde{v}_{\mathrm{abs}} \\
/ \mathrm{cm}^{-1}(\mathrm{~nm})\end{array}$ & $\begin{array}{c}\tilde{v}_{\mathrm{fl}} \\
/ \mathrm{cm}^{-1}(\mathrm{~nm})\end{array}$ & $\begin{array}{c}\tilde{v}_{\mathrm{fl}} \\
/ \mathrm{cm}^{-1}(\mathrm{~nm})\end{array}$ & $\phi_{\mathrm{f}}$ \\
\hline \multirow[t]{3}{*}{10} & $\mathrm{MeCN}$ & $38500(260)$ & 41900 (239) & $33000(303)$ & $34200(292)^{\mathrm{a}}$ & $-^{\mathrm{c}}$ \\
\hline & $\mathrm{CH}_{2} \mathrm{Cl}_{2}$ & $38400(260)$ & 41800 (239) & $32800(305)$ & $34000(294)^{\mathrm{a}}$ & $\sim 0.01$ \\
\hline & toluene & $-{ }^{b}$ & $-{ }^{b}$ & $29500(339)^{\mathrm{a}}$ & $30700(325)$ & $-^{\mathrm{c}}$ \\
\hline
\end{tabular}

${ }^{a}$ less intense maximum. ${ }^{b}$ could not be measured because of insufficient solvent transparency. ${ }^{c}$ could not be measured. 


\section{Crystal structure determinations}

The crystal datas were collected on a BRUKER X8-APEX II diffractometer with a CCD area detector and mirror monochromated $\mathrm{Mo}_{\mathrm{K} \alpha}$ radiation. The structures were solved using the intrinsic phasing method (SHELXT), refined with the SHELXLprogram ${ }^{6}$ and expanded using Fourier techniques. All non-hydrogen atoms were refined anisotropically. Hydrogen atoms were included in the structure factor calculations. All hydrogen atoms were assigned to idealized geometric positions. Crystallographic data have been deposited with the Cambridge Crystallographic Data Center as supplementary publication CCDC-1450301 (3), -1450302 (4), -1450303 (6), -1450304 (8), -1450305 (9), -1450306 (10), -1450307 (12). These data can be obtained free of charge from The Cambridge Crystallographic Data Centre via www.ccdc.cam.ac.uk/data_request/cif.

Crystal data for 3: $\mathrm{C}_{25} \mathrm{H}_{45} \mathrm{BClNPRh}, M_{\mathrm{r}}=539.76$, orange block, $0.42 \times 0.39 \times 0.38 \mathrm{~mm}^{3}$, monoclinic space group $P 21 / c, \quad a=11.910(2) \AA, \quad b=17.013(3) \AA, \quad c=14.078(2) \AA$, $\beta=109.423(5)^{\circ}, \quad V=2690.3(8) \AA^{3}, \quad Z=4, \quad \rho_{\text {calcd }}=1.333 \mathrm{~g} \cdot \mathrm{cm}^{-3}, \quad \mu=0.806 \mathrm{~mm}^{-1}$, $F(000)=1136, T=100(2) \mathrm{K}, R_{1}=0.0192, w R^{2}=0.0480,5499$ independent reflections $\left[2 \theta \leq 52.744^{\circ}\right]$ and 283 parameters.

Crystal data for 4: $\mathrm{C}_{18} \mathrm{H}_{26} \mathrm{BN}, M_{\mathrm{r}}=267.21$, colorless block, $0.27 \times 0.22 \times 0.07 \mathrm{~mm}^{3}$, triclinic space group $P-1, a=5.8630(7) \AA, b=14.7829(18) \AA, c=19.258(2) \AA, \alpha=104.809(4)^{\circ}$, $\beta=94.491(4)^{\circ}, \gamma=90.355(4)^{\circ}, V=1608.1(3) \AA^{3}, Z=4, \rho_{\text {calcd }}=1.104 \mathrm{~g} \cdot \mathrm{cm}^{-3}, \mu=0.062 \mathrm{~mm}^{-}$ ${ }^{1}, F(000)=584, T=100(2) \mathrm{K}, R_{l}=0.0578, w R^{2}=0.1267,6582$ independent reflections $\left[2 \theta \leq 52.74^{\circ}\right]$ and 373 parameters.

Crystal data for 6: $\mathrm{C}_{22} \mathrm{H}_{30} \mathrm{BFeN}, M_{\mathrm{r}}=375.13$, orange block, $0.30 \times 0.18 \times 0.06 \mathrm{~mm}^{3}$, triclinic space group $P-1, a=9.8819(5) \AA, b=10.5352(6) \AA, c=10.9353(6) \AA, \quad \alpha=99.896(2)^{\circ}$, $\beta=111.336(2)^{\circ}, \quad \gamma=107.565(2)^{\circ}, \quad V=958.78(9) \AA^{3}, \quad Z=2, \quad \rho_{\text {calcd }}=1.299 \mathrm{~g} \cdot \mathrm{cm}^{-3}$, $\mu=0.790 \mathrm{~mm}^{-1}, F(000)=400, T=100(2) \mathrm{K}, R_{l}=0.0288, w R^{2}=0.0698,3932$ independent reflections $\left[2 \theta \leq 52.74^{\circ}\right]$ and 232 parameters.

Crystal data for 8: $\mathrm{C}_{32} \mathrm{H}_{39} \mathrm{BN}_{2} \mathrm{O}_{2}, M_{\mathrm{r}}=494.46$, colorless needle, $0.218 \times 0.095 \times 0.086 \mathrm{~mm}^{3}$, triclinic space group $P \overline{1}, \quad a=10.050(2) \AA, \quad b=11.146(4) \AA, \quad c=12.973(4) \AA$, $\alpha=89.147(14)^{\circ}, \quad \beta=76.019(12)^{\circ}, \quad \gamma=88.928(13)^{\circ}, \quad V=1409.7(7) \AA^{3}, \quad Z=2$, 
$\rho_{\text {calcd }}=1.165 \mathrm{~g} \cdot \mathrm{cm}^{-3}, \quad \mu=0.071 \mathrm{~mm}^{-1}, \quad F(000)=532, \quad T=296(2) \mathrm{K}, \quad R_{1}=0.0828$, $w R^{2}=0.1361,6009$ independent reflections $\left[2 \theta \leq 53.624^{\circ}\right]$ and 342 parameters.

Crystal data for 9: $\mathrm{C}_{51} \mathrm{H}_{90} \mathrm{~B}_{1} \mathrm{Cl}_{2} \mathrm{~N}_{2} \mathrm{P}_{2} \mathrm{Rh}_{2}, M_{\mathrm{r}}=1081.91$, red block, $0.137 \times 0.122 \times 0.072 \mathrm{~mm}^{3}$, monoclinic space group $P 21 / n, \quad a=12.243(3) \AA, \quad b=16.865(4) \AA, \quad c=14.294(5) \AA$, $\beta=111.702(17)^{\circ}, \quad V=2742.3(14) \AA^{3}, \quad Z=2, \quad \rho_{\text {calcd }}=1.310 \mathrm{~g} \cdot \mathrm{cm}^{-3}, \quad \mu=0.791 \mathrm{~mm}^{-1}$, $F(000)=1140, T=100(2) \mathrm{K}, R_{1}=0.0213, w R^{2}=0.0506,5845$ independent reflections $\left[2 \theta \leq 53.524^{\circ}\right]$ and 283 parameters.

Crystal data for 10: $\mathrm{C}_{30} \mathrm{H}_{46} \mathrm{~B}_{2} \mathrm{~N}_{2}, M_{\mathrm{r}}=456.31$, colorless block, $0.23 \times 0.07 \times 0.03 \mathrm{~mm}^{3}$, monoclinic space group $C 2 / c, \quad a=26.975(13) \AA, \quad b=9.754(4) \AA, \quad c=11.280(7) \AA$, $\beta=114.33(3)^{\circ}, V=2704(2) \AA^{3}, Z=4, \rho_{\text {calcd }}=1.121 \mathrm{~g} \cdot \mathrm{cm}^{-3}, \mu=0.063 \mathrm{~mm}^{-1}, F(000)=1000$, $T=100(2) \mathrm{K}, R_{l}=0.0911, w R^{2}=0.1135,2901$ independent reflections $\left[2 \theta \leq 53.63^{\circ}\right]$ and 160 parameters.

Crystal data for 12: $\mathrm{C}_{42} \mathrm{H}_{66} \mathrm{~B}_{3} \mathrm{~N}_{3} \mathrm{O}_{0.15}, M_{\mathrm{r}}=647.80$, colorless block, $0.08 \times 0.07 \times 0.04 \mathrm{~mm}^{3}$, rhombohedral space group $R 3: H, \quad a=15.570(5) \AA, \quad b=15.570(5) \AA, \quad c=14.579(8) \AA$, $\alpha=90^{\circ}, \beta=90^{\circ}, \gamma=120^{\circ}, V=3061(3) \AA^{3}, Z=3, \rho_{\text {calcd }}=1.054 \mathrm{~g} \cdot \mathrm{cm}^{-3}, \mu=0.060 \mathrm{~mm}^{-1}$, $F(000)=1066, T=296(2) \mathrm{K}, R_{l}=0.0686, w R^{2}=0.1200,2800$ independent reflections $\left[2 \theta \leq 52.722^{\circ}\right]$ and 170 parameters. 


\section{Computational details for compound 8}

Calculation of the frontier molecular orbitals for $\mathbf{8}$ and Ref were carried out in the Gaussian09 software suite. ${ }^{7}$ The ground state molecular geometries were optimized at the B3LYP/6-311G(d) level of theory, and were found to be true minima through frequency analyses.

Table S2. Cartesian coordinates for the optimized geometry of $\mathbf{8}$.

\begin{tabular}{|c|c|c|c|}
\hline $\mathrm{O}$ & 5.08462674 & 4.92802266 & 0.83171861 \\
\hline $\mathrm{C}$ & -3.89267353 & 0.13324132 & -0.91930145 \\
\hline $\mathrm{H}$ & -3.21011032 & 0.36790456 & -1.73382032 \\
\hline $\mathrm{N}$ & -3.99764380 & -0.61870075 & 1.36687359 \\
\hline B & -5.38360342 & 0.42848732 & -0.99209255 \\
\hline $\mathrm{O}$ & 5.58602108 & -4.48449229 & -1.03594306 \\
\hline $\mathrm{N}$ & 2.46336314 & 0.04053684 & 0.03974187 \\
\hline $\mathrm{C}$ & -3.26250969 & -0.31134282 & 0.21823284 \\
\hline $\mathrm{C}$ & -5.32794223 & -0.25211810 & 1.40396140 \\
\hline $\mathrm{H}$ & -5.79814162 & -0.41539168 & 2.36333187 \\
\hline $\mathrm{C}$ & -6.11713665 & 0.91562042 & -2.33685185 \\
\hline $\mathrm{C}$ & -6.04200623 & 0.26169729 & 0.36413908 \\
\hline $\mathrm{H}$ & -7.07781942 & 0.50362513 & 0.59316681 \\
\hline $\mathrm{C}$ & -5.91670203 & -0.14469009 & -3.44378898 \\
\hline $\mathrm{H}$ & -6.38549132 & 0.17443301 & -4.38387430 \\
\hline $\mathrm{H}$ & -6.36158733 & -1.10660381 & -3.16755308 \\
\hline $\mathrm{H}$ & -4.85700658 & -0.32331284 & -3.64934621 \\
\hline $\mathrm{C}$ & -5.49334171 & 2.24697295 & -2.81621671 \\
\hline $\mathrm{H}$ & -5.96796465 & 2.58887647 & -3.74534689 \\
\hline $\mathrm{H}$ & -4.42148877 & 2.15011134 & -3.01304856 \\
\hline $\mathrm{H}$ & -5.61789464 & 3.04262813 & -2.07411398 \\
\hline $\mathrm{C}$ & -7.62870415 & 1.13239614 & -2.13682621 \\
\hline $\mathrm{H}$ & -8.11137837 & 1.45237266 & -3.06898855 \\
\hline $\mathrm{H}$ & -7.83271193 & 1.90279021 & -1.38592185 \\
\hline $\mathrm{H}$ & -8.13007145 & 0.21582180 & -1.80881820 \\
\hline $\mathrm{C}$ & -3.52779384 & -1.46896705 & 2.55116921 \\
\hline $\mathrm{C}$ & -2.23935786 & -2.26147331 & 2.27210535 \\
\hline $\mathrm{H}$ & -2.09878212 & -2.96360315 & 3.09733013 \\
\hline $\mathrm{H}$ & -1.34879577 & -1.64403203 & 2.21858419 \\
\hline $\mathrm{H}$ & -2.31314265 & -2.84461601 & 1.35275307 \\
\hline $\mathrm{C}$ & -4.60942925 & -2.53513280 & 2.85740083 \\
\hline $\mathrm{H}$ & -4.22091026 & -3.23392139 & 3.60098100 \\
\hline $\mathrm{H}$ & -4.85896183 & -3.10279991 & 1.95817148 \\
\hline $\mathrm{H}$ & -5.53237250 & -2.12396731 & 3.26453723 \\
\hline $\mathrm{C}$ & -1.76969881 & -0.28753304 & 0.23496896 \\
\hline $\mathrm{C}$ & -1.02123619 & -1.07557404 & -0.64542519 \\
\hline $\mathrm{H}$ & -1.53356077 & -1.78053589 & -1.29241609 \\
\hline $\mathrm{C}$ & 0.36246955 & -0.97392345 & -0.71387963 \\
\hline $\mathrm{H}$ & 0.91181193 & -1.59704920 & -1.40977791 \\
\hline $\mathrm{C}$ & 1.05795748 & -0.06611756 & 0.10137808 \\
\hline $\mathrm{C}$ & 0.30858305 & 0.74178307 & 0.97366344 \\
\hline $\mathrm{H}$ & 0.81596402 & 1.46410776 & 1.60235070 \\
\hline $\mathrm{C}$ & -1.07518075 & 0.63613933 & 1.02646426 \\
\hline $\mathrm{H}$ & -1.62713783 & 1.29280289 & 1.69134351 \\
\hline $\mathrm{C}$ & 3.26327388 & -1.09936876 & -0.26411221 \\
\hline $\mathrm{C}$ & 3.06993988 & -2.31316844 & 0.41405543 \\
\hline $\mathrm{H}$ & 2.29713881 & -2.38286922 & 1.17149589 \\
\hline $\mathrm{C}$ & 3.85063824 & -3.41960063 & 0.12504382 \\
\hline
\end{tabular}




$\begin{array}{lrrr}\mathrm{H} & 3.70374172 & -4.35894078 & 0.64656307 \\ \mathrm{C} & 4.86685099 & -3.34224009 & -0.83617032 \\ \mathrm{C} & 5.07737722 & -2.13572178 & -1.50716569 \\ \mathrm{H} & 5.85325860 & -2.03941214 & -2.25622098 \\ \mathrm{C} & 4.27165847 & -1.03197792 & -1.22613989 \\ \mathrm{H} & 4.43763186 & -0.10264311 & -1.75953550 \\ \mathrm{C} & 6.62623046 & -4.46711353 & -1.99936360 \\ \mathrm{H} & 7.05632138 & -5.46701335 & -1.99070944 \\ \mathrm{H} & 7.40261460 & -3.73832553 & -1.74108244 \\ \mathrm{H} & 6.24309338 & -4.24796075 & -3.00214495 \\ \mathrm{C} & 3.10469789 & 1.30022755 & 0.22533975 \\ \mathrm{C} & 4.22458330 & 1.41250027 & 1.06348933 \\ \mathrm{H} & 4.59495508 & 0.53290541 & 1.57822617 \\ \mathrm{C} & 4.86398174 & 2.62919175 & 1.23362893 \\ \mathrm{H} & 5.73039359 & 2.71944501 & 1.87944017 \\ \mathrm{C} & 4.39157211 & 3.77829857 & 0.58705760 \\ \mathrm{C} & 3.27301662 & 3.67975010 & -0.24377242 \\ \mathrm{H} & 2.88404226 & 4.54543484 & -0.76501528 \\ \mathrm{C} & 2.64856960 & 2.44614860 & -0.42818300 \\ \mathrm{H} & 1.78886190 & 2.37905475 & -1.08568207 \\ \mathrm{C} & 4.65172369 & 6.12298728 & 0.20298723 \\ \mathrm{H} & 5.33418382 & 6.90087181 & 0.54063464 \\ \mathrm{H} & 3.62997572 & 6.38621264 & 0.49836314 \\ \mathrm{H} & 4.70440034 & 6.04610900 & -0.88873620 \\ \mathrm{C} & -3.32142625 & -0.55783780 & 3.77147582 \\ \mathrm{H} & -3.06176403 & -1.15532901 & 4.64974635 \\ \mathrm{H} & -4.22471094 & 0.00848188 & 4.00966385 \\ \mathrm{H} & -2.51169441 & 0.15248480 & 3.59723446\end{array}$




\section{Computational details for mechanistic study}

\section{Breakdown of Energy Contributions}

The following tables detail the evolution of the relative energies as the successive corrections to the initial SCF energy are included. Terms used are:

$\Delta \mathbf{E} \quad$ SCF energy computed with the BP86 functional

$\Delta \mathbf{H} \quad$ Enthalpy at $0 \mathrm{~K}$

$\Delta \mathbf{G} \quad$ Free energy at $298.15 \mathrm{~K}$ and $1 \mathrm{~atm}$

$\Delta \mathbf{G}_{\mathbf{C 6 H 6}} \quad$ Free energy corrected for benzene solvent

$\Delta \mathbf{G}_{\mathbf{D} 3} \quad$ Free energy corrected for dispersion effects

$\Delta \mathbf{G}_{\text {final }} \quad$ Free energy corrected for benzene solvent and dispersion effects

$\Delta \mathbf{G}_{\text {final }}$ is the final data used in the main article 
Scheme S1: Stationary Points for the reaction of $\mathbf{A 1}$ to 3 where $\mathrm{L}={ }^{\mathrm{i}} \mathrm{Pr}_{3}$. Alternative pathways are also indicated. $\Delta \mathbf{G}_{\text {final }}$ quoted in $\mathrm{kcal} / \mathrm{mol}$.

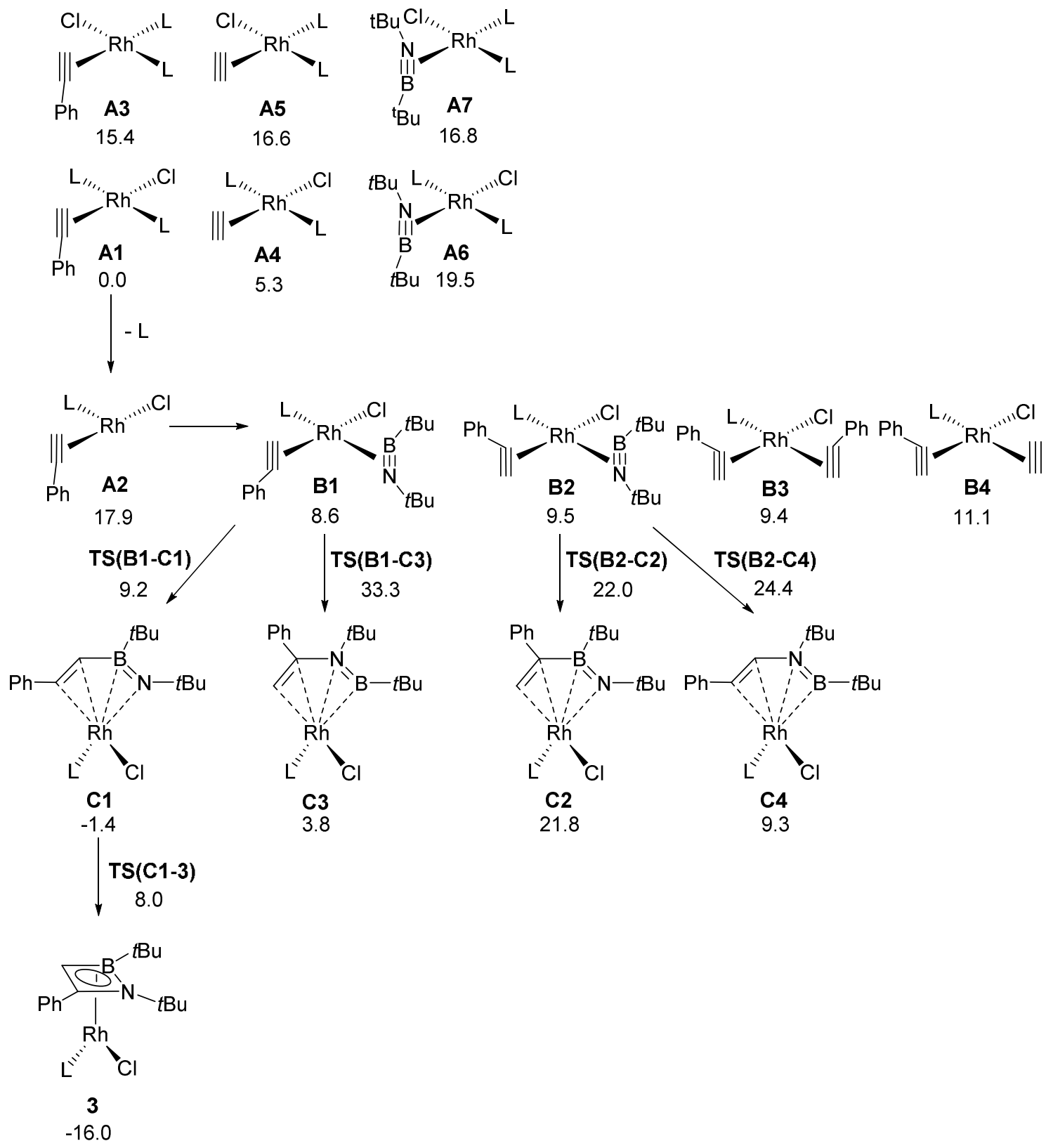


Scheme S2: Alternative pathways for the reaction of $\mathbf{3}$ to $\mathbf{G}$ where $\mathrm{L}=\mathrm{P}^{\mathrm{i}} \operatorname{Pr}_{3} . \Delta \mathbf{G}_{\text {final }}$ quoted in $\mathrm{kcal} / \mathrm{mol}$. No transition states were located for (i) $\mathrm{C}-\mathrm{C}$ bond cleavage in 3; (ii) B-C bond cleavage in D1; (iii) C-C bond cleavage in D1; and (iv) insertion of acetylene into the Rh-N bond of D1

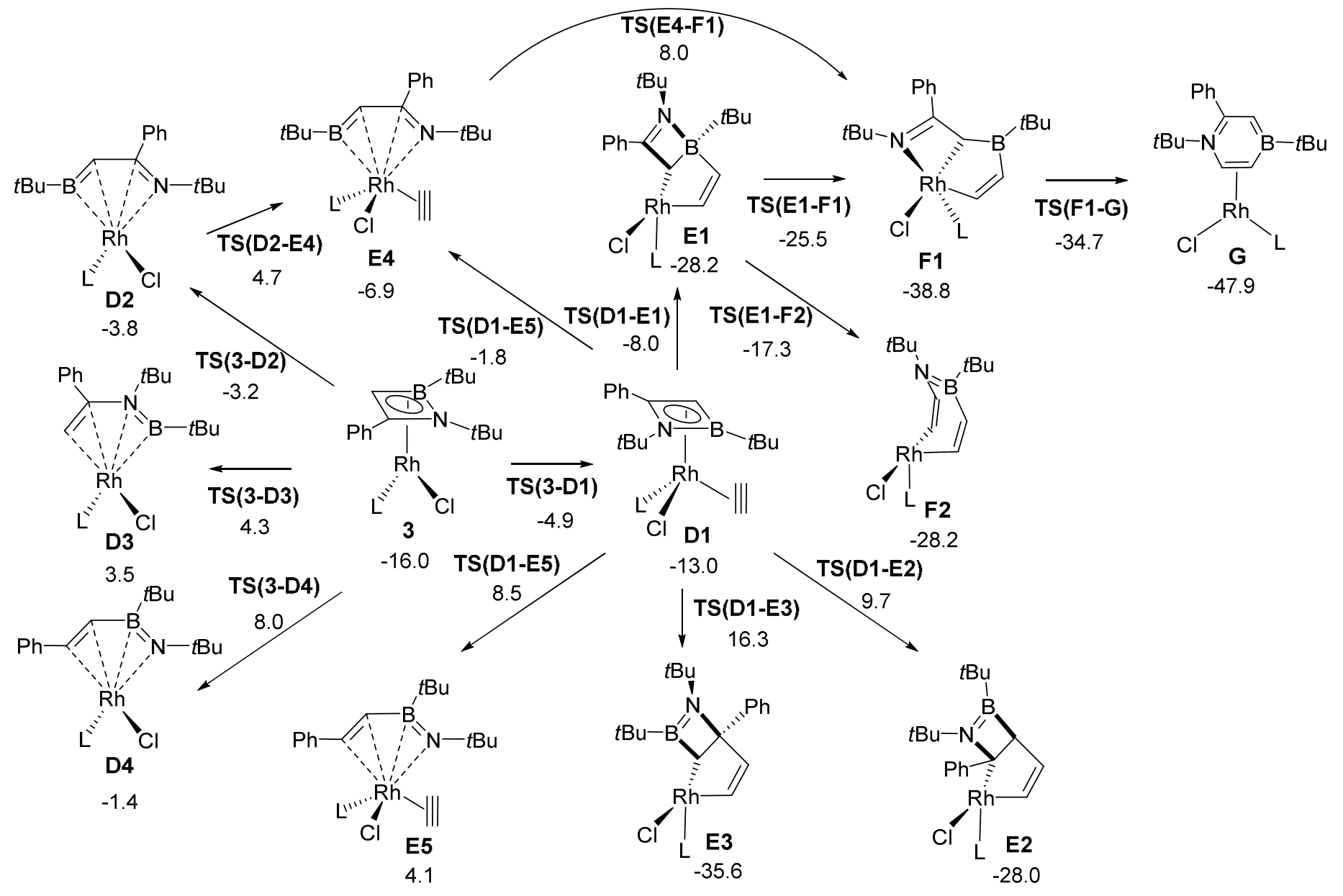


Table S3: Computed relative energies $(\mathrm{kcal} / \mathrm{mol})$ for all intermediates and transition states. All energies are quoted relative to $\mathbf{A 1}$ set to $0.0 \mathrm{kcal} / \mathrm{mol}$.

\begin{tabular}{|c|c|c|c|c|c|c|}
\hline Species & $\Delta \mathbf{E}$ & $\Delta \mathbf{H}$ & $\Delta \mathbf{G}$ & $\Delta \mathrm{G}_{\mathrm{C6H6}}$ & $\Delta \mathbf{G}_{\mathrm{D} 3}$ & $\Delta \mathbf{G}_{\text {final }}$ \\
\hline A1 & 0.0 & 0.0 & 0.0 & 0.0 & 0.0 & 0.0 \\
\hline $\mathbf{A 2}$ & 16.6 & 15.3 & 0.9 & -1.7 & 20.5 & 17.9 \\
\hline A3 & 18.6 & 18.6 & 19.2 & 17.6 & 17.1 & 15.4 \\
\hline A4 & -0.3 & 1.5 & -2.6 & -2.6 & 5.1 & 5.3 \\
\hline A5 & 17.6 & 18.8 & 14.0 & 12.2 & 18.4 & 16.6 \\
\hline A6 & 27.3 & 28.0 & 32.2 & 32.5 & 19.2 & 19.5 \\
\hline $\mathbf{A} 7$ & 26.2 & 26.9 & 31.4 & 31.2 & 17.0 & 16.8 \\
\hline B1 & 8.5 & 8.1 & 8.8 & 8.3 & 9.2 & 8.6 \\
\hline B2 & 10.6 & 9.9 & 9.8 & 9.5 & 9.5 & 9.5 \\
\hline B3 & 3.4 & 2.9 & 0.7 & 0.0 & 10.1 & 9.4 \\
\hline B4 & 3.6 & 4.5 & -1.9 & -2.9 & 12.4 & 11.1 \\
\hline TS(B1-C1) & 9.3 & 8.3 & 9.5 & 8.9 & 9.5 & 9.2 \\
\hline TS(B1-C3) & 33.9 & 32.9 & 35.2 & 34.1 & 34.1 & 33.3 \\
\hline TS(B2-C2) & 22.8 & 22.2 & 24.2 & 23.6 & 22.6 & 22.0 \\
\hline TS(B2-C4) & 34.7 & 34.4 & 36.9 & 36.2 & 35.1 & 34.4 \\
\hline C1 & -5.2 & -4.3 & -1.4 & -1.8 & -1.0 & -1.4 \\
\hline $\mathrm{C} 2$ & 20.6 & 20.8 & 23.2 & 22.4 & 22.6 & 21.8 \\
\hline C3 & 2.5 & 3.6 & 6.3 & 4.9 & 5.2 & 3.8 \\
\hline $\mathrm{C} 4$ & 10.6 & 9.9 & 9.8 & 9.4 & 9.7 & 9.3 \\
\hline TS(C1-3) & 4.2 & 4.4 & 7.0 & 6.5 & 8.5 & 8.0 \\
\hline 3 & -18.4 & -16.4 & -13.2 & -13.8 & -15.7 & -16.0 \\
\hline TS(3-D1) & -7.3 & -4.5 & 4.7 & 4.9 & -5.1 & -4.9 \\
\hline TS(3-D2) & -3.8 & -3.4 & -1.1 & -1.9 & -2.4 & -3.2 \\
\hline TS(3-D3) & 3.5 & 4.4 & 7.9 & 6.9 & 5.4 & 4.3 \\
\hline TS(3-D4) & 4.2 & 4.4 & 7.0 & 6.5 & 8.5 & 8.0 \\
\hline D1 & -17.1 & -12.7 & -1.2 & -0.8 & -13.4 & -13.0 \\
\hline D2 & -9.2 & -7.7 & -5.0 & -5.7 & -3.1 & -3.8 \\
\hline D3 & 1.7 & 2.9 & 5.4 & 5.0 & 3.9 & 3.5 \\
\hline D4 & -5.2 & -4.3 & -1.4 & -1.8 & -1.0 & -1.4 \\
\hline TS(D1-E1) & -12.8 & -8.9 & 3.3 & 3.9 & -8.7 & -8.0 \\
\hline
\end{tabular}




\begin{tabular}{ccccccc} 
TS(D1-E2) & 6.1 & 9.8 & 20.8 & 20.5 & 10.0 & 9.7 \\
TS(D1-E3) & 11.9 & 14.8 & 25.0 & 22.6 & 18.7 & 16.3 \\
TS(D1-E4) & -3.6 & -0.4 & 10.6 & 10.1 & -1.3 & -1.8 \\
TS(D1-E5) & 5.0 & 7.6 & 18.8 & 18.8 & 8.5 & 8.5 \\
TS(D2-E4) & 2.1 & 4.5 & 13.6 & 13.3 & 4.9 & 4.7 \\
E1 & -33.1 & -28.1 & -17.5 & -18.2 & -27.4 & -28.2 \\
E2 & -37.8 & -31.9 & -20.6 & -20.9 & -27.8 & -28.0 \\
E3 & -43.5 & -37.6 & -27.1 & -28.0 & -34.7 & -35.6 \\
E4 & -10.6 & -6.6 & 4.1 & 4.1 & -6.9 & -6.9 \\
E5 & 0.3 & 3.6 & 15.3 & 15.7 & 3.7 & 4.1 \\
TS(E1-F1) & -31.7 & -27.0 & -14.9 & -15.1 & -25.3 & -25.5 \\
TS(E1-F2) & -21.8 & -17.0 & -5.0 & -5.9 & -16.5 & -17.3 \\
TS(E4-F1) & 0.0 & 3.6 & 15.0 & 15.7 & 7.3 & 8.0 \\
F1 & -49.4 & -44.0 & -33.2 & -33.2 & -38.8 & -38.8 \\
F2 & -33.1 & -28.1 & -17.5 & -18.2 & -27.4 & -28.2 \\
TS(F1-G) & -34.1 & -28.7 & -17.9 & -17.6 & -23.4 & -34.7 \\
G & -58.3 & -51.1 & -40.4 & -40.5 & -47.9 & -47.9 \\
\hline & & & & & & \\
\hline & & & & & & \\
\hline
\end{tabular}




\section{Functional Testing}

Table S4: Single point calculations for key transition states and intermediates with selected functionals. Results displayed are computed relative free energies, $\Delta \mathrm{G}$ in $\mathrm{kcal} / \mathrm{mol}$. The results show consistency in preference of TS(B1-C1) over TS(B2-C2) and TS(D1-E1) over TS(D1-E2) for every functional tested.

\begin{tabular}{ccccccc}
\hline Species & BP86 & B3LYP & M06 & PBE0 & B97D & WB97XD \\
\hline A1 & 0.0 & 0.0 & 0.0 & 0.0 & 0.0 & 0.0 \\
B1 & 0.6 & 14.6 & 14.7 & 14.0 & 10.8 & 12.2 \\
TS(B1-C1) & 9.5 & 14.4 & 15.2 & 12.4 & 9.5 & 11.3 \\
C1 & -1.4 & 9.5 & 12.4 & 6.4 & 3.9 & 8.0 \\
B2 & 9.8 & 12.0 & 10.2 & 11.9 & 8.0 & 9.1 \\
TS(B2-C2) & 24.2 & 30.5 & 27.9 & 27.4 & 22.8 & 25.1 \\
C2 & 23.2 & 30.8 & 29.2 & 27.6 & 23.0 & 25.4 \\
3 & -13.2 & -8.4 & -15.2 & -18.5 & -15.4 & -23.0 \\
TS(D1-E1) & 3.3 & 18.3 & 5.2 & -1.6 & 0.7 & -3.2 \\
TS(D1-E4) & 10.6 & 23.0 & 12.8 & 8.1 & 5.7 & 5.2 \\
TS(E4-F1) & 15.0 & 27.3 & 20.2 & 12.6 & 14.4 & 11.7 \\
G & -40.4 & -34.4 & -43.9 & -50.1 & -45.7 & -54.4 \\
\hline
\end{tabular}




\title{
Cartesian Coordinates ( $\AA$ ) and computed energies (in hartrees) for all optimized
} structures. All structures can be visualized via the Mechanism.xyz file provided.

\author{
$\left[\mathrm{Rh}\left(\mathrm{P}^{\mathrm{i}} \mathrm{Pr}_{3}\right)_{2} \mathrm{Cl}\right]_{2}(2)$ \\ SCF Energy $=-1700.33670847$ \\ Enthalpy $0 \mathrm{~K}=-1699.216785$ \\ Enthalpy $298 \mathrm{~K}=-1699.145996$ \\ Free Energy 298K = -1699.317617 \\ $\mathrm{SCF}(\mathrm{C} 6 \mathrm{H} 6)=-1700.34042713$ \\ SCF $(\mathrm{BP} 86-\mathrm{D} 3)=-0.5948694$ \\ Lowest Frequencies $=9.113123 .8004 \mathrm{~cm}-1$ \\ C $-1.88903 \quad 3.215432 .16660$ \\ C -3.284972 .697681 .78969$ \\ P -3.354661 .811210 .08537$ \\ C $-2.712303 .18165-1.09953$ \\ C $-3.464124 .52496-1.09729$ \\ C $\begin{array}{llll}-3.81650 & 1.77130 & 2.89780\end{array}$ \\ C $-5.26479 \quad 1.73981-0.16653$ \\ C -6.111752 .898120 .40097$ \\ Rh - $1.95066-0.000020 .00002$ \\ P $-3.35464-1.81123-0.08536$ \\ C $-5.26476-1.739720 .16656$ \\ C $-5.62522-1.483661 .64163$ \\ C $-5.62526 \quad 1.48389-1.64162$ \\ Cl $0.00001 \quad 1.52052-0.20104$ \\ $\mathrm{Rh} 1.950650 .00003-0.00003$ \\ P $3.35460-1.81132-0.08456$ \\ C $2.71225-3.18117 \quad 1.10103$ \\ C $3.46423-4.524391 .09961$ \\ $\mathrm{Cl} \quad 0.00002-1.52050 \quad 0.20094$ \\ P $3.35463 \quad 1.811280 .08455$ \\ C $5.264731 .73959-0.16755$ \\ C $5.624901 .48271-1.64254$ \\ C $2.712293 .18125-1.10090$ \\ C $2.49103 \quad 2.65622-2.53294$ \\ C $3.28524 \quad 2.69861 \quad 1.78845$ \\ C $1.889373 .21657 \quad 2.16535$ \\ C $3.464314 .52445-1.09940$ \\ C $-2.71235-3.18170 \quad 1.09951$ \\ C $-2.49141-2.657312 .53183$ \\ C $-3.28505-2.69771-1.78969$ \\ C $-1.88912-3.21546-2.16666$ \\ C $-3.46419-4.52500 \quad 1.09726$ \\ C $-2.491382 .65722-2.53183$ \\ C $-3.81665-1.77135-2.89777$ \\ C $-6.11179-2.89804-0.40084$ \\ C $5.26471-1.739730 .16745$ \\ C $6.11177-2.89834-0.39929$ \\ C $3.28507-2.69865-1.78843$ \\ C $1.88916-3.21652-2.16531$ \\ C $5.62497-1.482881 .64242$ \\ C $3.81678-1.77281-2.89690$ \\ C $2.49114-2.655992 .53303$ \\ C 3.816921 .772762 .89692 \\ $\begin{array}{llll}\text { C } & 6.11191 & 2.89814 & 0.39913\end{array}$ \\ H -3.96316 3.565431 .69056 \\ $\mathrm{H}-1.173832 .384082 .26473$ \\ H $-1.478963 .92127 \quad 1.42652$ \\ H -1.945213 .742683 .13752$ \\ $\mathrm{H}-4.85623 \quad 1.44650 \quad 2.72341$ \\ H -3.183320 .870102 .97255$
}


H -3.792912 .292253 .87305$

H $-1.707313 .35310-0.67326$

H $-4.44428 \quad 4.45404-1.59940$

H $-3.626054 .92752-0.08320$

H $-2.87315 \quad 5.27499-1.65622$

H - $1.921441 .71352-2.52081$

H $-3.441372 .48619-3.06500$

H $-1.914783 .40079-3.11332$

$\begin{array}{llll}\mathrm{H} & -5.53159 & 0.84025 & 0.41373\end{array}$

H $-6.007593 .01071 \quad 1.49150$

H $-5.872583 .86541-0.06683$

H $-7.179602 .69256 \quad 0.19743$

H $-5.532532 .40573-2.24059$

H $-4.976490 .72056-2.09991$

H $-6.674681 .14576-1.72892$

H $-3.96324-3.56546-1.69050$

H - $1.17391-2.38412-2.26471$

H - $1.47908-3.92137-1.42662$

H - $1.94531-3.74263-3.13761$

H -4.85637-1.44656 -2.72333

H -3.18349 - $0.87013-2.97255$

H -3.79309-2.29229 -3.87303

H -5.53152 - $0.84020-0.41377$

H $-5.53251-2.405462 .24067$

H $-4.97641-0.720312 .09985$

H -6.67462 -1.14549 1.72893

H -6.00767-3.01070-1.49136

H $-5.87263-3.865300 .06701$

H -7.17963 -2.69243 - 0.19728

H $-1.70735-3.353190 .67328$

H - $1.92111-1.713832 .52083$

H $-3.44138-2.485893 .06491$

H -1.91515 -3.40109 3.11340

H $-4.44430-4.454111 .59955$

H $-3.62631-4.927440 .08316$

H $-2.87315-5.275081 .65603$

H $3.96322-3.56639-1.68880$

H $1.70732-3.35297 \quad 0.67478$

H $5.53152-0.84050-0.41333$

H 3.963423 .566311 .68876

$\begin{array}{lllll}\mathrm{H} & 5.53151 & 0.84035 & 0.41323\end{array}$

H $1.707383 .35309-0.67459$

H $1.17411-2.38514-2.26422$

H $1.47881-3.92174-1.42479$

H $1.94552-3.74449-3.13582$

H $4.85658-1.44816-2.72266$

H $3.18377-0.87151-2.97193$

H $3.79305-2.29410-3.87197$

H $4.44432-4.453081 .60181$

H $3.62634-4.927470 .08576$

H $2.87328-5.27418 \quad 1.65888$

H $1.92110-1.712362 .52146$

H $3.44106-2.484573 .06621$

H $1.91457-3.399323 .11486$

H $6.00766-3.01160-1.48975$

H $5.87265-3.865360 .06908$

H $7.17960-2.69257-0.19584$

H $5.53217-2.404352 .24195$

H $4.97608-0.719292 .10013$

H $6.67436-1.144661 .72969$

H $1.17422 \quad 2.38527 \quad 2.26407$

H 1.479173 .921981 .42492 
H $1.94573 \quad 3.744373 .13595$

H $4.85668 \quad 1.448022 .72265$

$\begin{array}{llll}\mathrm{H} & 3.18385 & 0.87151 & 2.97202\end{array}$

H 3.793262 .294093 .87197

H $5.532152 .40419-2.24206$

H $4.975910 .71918-2.10019$

H $6.674261 .14440-1.72986$

H 6.007923 .011391 .48960

H $5.872793 .86519-0.06920$

H 7.179712 .692330 .19556

H $1.920481 .71290-2.52142$

H $3.440892 .48430-3.06606$

H $1.914913 .39989-3.11478$

H $4.444474 .45312-1.60152$

H $3.626354 .92751-0.08554$

H $2.873435 .27425-1.65873$

$\left[\mathrm{Rh}\left(\mathrm{P}^{\mathrm{i}} \mathrm{Pr}_{3}\right)_{2} \mathrm{Cl}\right]$

SCF Energy $=-850.149148992$

Enthalpy $0 \mathrm{~K}=-849.591352$

Enthalpy $298 \mathrm{~K}=-849.555991$

Free Energy $298 \mathrm{~K}=-849.654250$

$\mathrm{SCF}(\mathrm{C} 6 \mathrm{H} 6)=-850.155704$

SCF $(B P 86-D 3)=-0.11277149$

Lowest Frequencies $=26.968834 .7814 \mathrm{~cm}-1$

C $-1.904160 .58451 \quad 1.90442$

$\begin{array}{llll}\mathrm{P} & -1.67409 & 0.28507 & 0.01862\end{array}$

C $-1.507602 .00641-0.81051$

$\mathrm{Rh} 0.08841-1.08499-0.21121$

P $1.904910 .25805-0.00196$

C $3.21831-1.13131-0.22458$

$\mathrm{Cl}-1.15580-3.08928-0.33876$

C $-3.29246-0.53464-0.58627$

$\begin{array}{llll}\text { C } & 2.34689 & 1.09763 & 1.65851\end{array}$

C $2.460411 .55752-1.30675$

$\begin{array}{llll}C & -2.72027 & 1.79689 & 2.39138\end{array}$

C $-2.35626-0.727632 .57766$

H $-0.85348 \quad 0.754972 .20036$

H $-3.19023-1.52887-0.11579$

C $-4.618540 .09806-0.12523$

C -3.27293 $-0.76052-2.10929$

C $-2.737962 .93636-0.81534$

H $-0.710562 .46230-0.18814$

$\begin{array}{llll}\text { C } & -0.97032 & 1.86108 & -2.24819\end{array}$

H $3.43858 \quad 1.27476 \quad 1.62897$

$\begin{array}{llll}\text { C } & 1.63399 & 2.45187 & 1.82937\end{array}$

C $2.02587 \quad 0.156682 .83445$

H $1.645772 .30604-1.27770$

C $3.796572 .26883-1.02287$

C $2.47657 \quad 0.92652-2.71455$

H $3.47104-1.05711-1.29776$

C $4.52666-1.056930 .57799$

C $2.47817-2.46928-0.01960$

H $-4.09269-1.44786-2.38620$

H $-3.427890 .17913-2.66770$

H -2.32646-1.22223 -2.43294

H $-5.45219-0.54610-0.46157$

$\begin{array}{llll}\mathrm{H} & -4.69438 & 0.17705 & 0.97021\end{array}$

H $-4.78997 \quad 1.09854-0.55328$

H -2.24090 -0.640663 .67344$

H -3.41844 -0.94892 2.37713

H $-1.75811-1.586362 .22820$ 
H $-2.72323 \quad 1.80415 \quad 3.49735$

H -2.284612 .755032 .06381$

H $-3.77257 \quad 1.767882 .06532$

H -2.43269 $3.93917-1.16795$

H $-3.502882 .57124-1.52057$

H $-3.211293 .05998 \quad 0.16846$

H $-0.549102 .82113-2.59942$

H $-0.19656 \quad 1.08124-2.30745$

H $-1.77795 \quad 1.58248-2.94479$

H $2.44701 \quad 1.71349-3.48885$

H $3.401540 .34629-2.87969$

H $1.622240 .24755-2.87804$

H $3.996583 .02095-1.80828$

H $3.812522 .79383-0.05440$

H $4.639411 .55673-1.03777$

H 1.931622 .917842 .78647

H $1.872803 .16731 \quad 1.02515$

H $\quad \begin{array}{llll}0.53770 & 2.32792 & 1.85512\end{array}$

H 2.264870 .649943 .79476

H $0.95785-0.120482 .83974$

H $2.59920-0.783062 .78952$

H $5.19159-1.88813 \quad 0.27767$

H $5.07805-0.117690 .40676$

H $4.35786-1.15641 \quad 1.66320$

H $3.11659-3.33240-0.28817$

H $2.13840-2.613381 .01957$

H $1.59531-2.56417-0.70506$

Intermediate A1

SCF Energy $=-1158.57662162$

Enthalpy $0 \mathrm{~K}=-1157.910959$

Enthalpy $298 \mathrm{~K}=-1157.867368$

Free Energy 298K $=-1157.985045$

$\mathrm{SCF}(\mathrm{C} 6 \mathrm{H} 6)=-1158.580614$

$\mathrm{SCF}(\mathrm{BP} 86-\mathrm{D} 3)=-0.13858626$

Lowest Frequencies $=12.633320 .1585 \mathrm{~cm}-1$

C $\quad 0.09530 \quad 1.48515-0.83153$

C $-0.009290 .63563-1.79541$

Rh $-0.05617-0.46339-0.05808$

Cl $-0.16508-2.26157 \quad 1.53301$

P $2.35425-0.72618-0.08991$

C $3.32786 \quad 0.81077-0.70866$

C $3.283250 .95938-2.24412$

C $2.84731-2.14718-1.27402$

C $2.19670-3.47802-0.85061$

C $4.34612-2.35134-1.57539$

C $2.97503-1.156201 .66108$

$\begin{array}{llll}\text { C } & 2.68709 & 0.02640 & 2.60759\end{array}$

C $4.38772-1.737191 .85426$

C $4.774350 .97999-0.20172$

P $-2.48590-0.43857-0.07255$

C $-3.232420 .55789-1.53432$

C $-3.225102 .07488-1.25497$

C $-3.03359-2.25793-0.26251$

C $-2.50805-2.80372-1.60578$

$\begin{array}{llll}C & -3.31232 & 0.28495 & 1.49991\end{array}$

C $-2.90058-0.494702 .76289$

C $-4.50232-2.63665-0.00534$

$\begin{array}{llll}C & -4.83943 & 0.50088 & 1.47277\end{array}$

$\begin{array}{llll}\text { C } & -4.60151 & 0.11341 & -2.08554\end{array}$

H $2.24478-1.945001 .92135$

H $2.72687 \quad 1.63731-0.28609$ 
H $2.35320-1.81310-2.20756$

H $-0.096450 .41666-2.85688$

$\begin{array}{lllll}\text { C } & 0.26702 & 2.80481 & -0.27543\end{array}$

H $-2.83017 \quad 1.28117 \quad 1.54571$

H $-2.41788-2.720760 .53154$

H $-2.477850 .37203-2.32202$

H $-4.86898 \quad 0.75519-2.94624$

H $-4.59661-0.92690-2.44524$

H $-5.410590 .21227-1.34375$

H $-3.444562 .62058-2.19104$

H $-3.997792 .36436-0.52359$

H - $2.252922 .42843-0.88258$

H -2.62317 -3.90243 - 1.63285

H -3.05467 -2.39351-2.47296

H - $1.43623-2.56951-1.73213$

H - $4.61903-3.72823-0.14241$

H $-4.80993-2.40874 \quad 1.02750$

H -5.20931 -2.14396-0.69064

H $-5.14218 \quad 1.025342 .39827$

$\begin{array}{llll}\mathrm{H} & -5.17762 & 1.11567 & 0.62527\end{array}$

H $-5.39361-0.450451 .44469$

H $-3.254420 .04633 \quad 3.65987$

H -3.35581 -1.50009 2.78158

H $-1.81126-0.630742 .83227$

H $3.693121 .94681-2.52486$

H $3.89516 \quad 0.19728-2.75451$

$\begin{array}{llll}\mathrm{H} & 2.25632 & 0.89813 & -2.63338\end{array}$

H $5.18245 \quad 1.93144-0.59153$

$\begin{array}{lllll}\mathrm{H} & 4.83932 & 1.02479 & 0.89584\end{array}$

H $5.44003 \quad 0.17332-0.55028$

H $2.85499-0.287503 .65384$

$\begin{array}{lllll}\mathrm{H} & 3.33578 & 0.89861 & 2.41564\end{array}$

$\begin{array}{lllll}\mathrm{H} & 1.63688 & 0.35063 & 2.51799\end{array}$

H $4.54325-1.935392 .93147$

H $4.51269-2.698151 .33134$

H $5.19311-1.061681 .52725$

H $2.36173-4.23605-1.63834$

H $2.64076-3.86763 \quad 0.08191$

H $1.11506-3.37479-0.67719$

H $4.45296-3.11565-2.36764$

H $4.84816-1.43920-1.93187$

H $4.89823-2.71793-0.69619$

C $0.507193 .92463-1.11946$

C $0.669565 .20273-0.57406$

$\begin{array}{llll}\text { C } & 0.59797 & 5.39966 & 0.81783\end{array}$

$\begin{array}{llll}\text { C } & 0.35941 & 4.30203 & 1.66245\end{array}$

C $0.19462 \quad 3.01848 \quad 1.12554$

H $\quad 0.563353 .77264-2.20231$

H $0.854606 .05395-1.23843$

$\begin{array}{lllll}\mathrm{H} & 0.72668 & 6.40217 & 1.23865\end{array}$

H 0.300614 .447262 .74658

$\begin{array}{lllll}\mathrm{H} & 0.00829 & 2.15303 & 1.77051\end{array}$

Intermediate A2

SCF Energy $=-796.377981246$

Enthalpy 0K $=-795.990424$

Enthalpy $298 \mathrm{~K}=-795.963246$

Free Energy 298K = -796.046879

SCF $(\mathrm{C} 6 \mathrm{H} 6)=-796.384921888$

SCF (BP86-D3) $=-0.07364945$

Lowest Frequencies $=22.263125 .1440 \mathrm{~cm}-1$

C -1.76296 -0.72671 -0.95001 
C $-1.05344-0.98977-2.01211$

Rh $0.09730-1.37689-0.42264$

Cl $1.49454-2.805240 .80702$

$\begin{array}{llll}\text { P } & 1.29280 & 0.46801 & -0.01940\end{array}$

C $0.21938 \quad 2.03428-0.25721$

C $-0.076272 .30912-1.74441$

$\begin{array}{llll}\text { C } & 1.96723 & 0.43528 & 1.76458\end{array}$

C $0.79736 \quad 0.45222 \quad 2.76711$

C $3.08156 \quad 1.429762 .14035$

C $2.766010 .56927-1.23249$

C $3.77023-0.57025-0.97191$

C $3.479031 .93299-1.34026$

$\begin{array}{llll}\text { C } & 0.70398 & 3.31081 & 0.45935\end{array}$

C $-2.96723-0.21930-0.32558$

H - $0.96664-1.02540-3.09644$

H $2.259140 .36473-2.19473$

H $2.38826-0.587331 .77986$

$\begin{array}{llll}\mathrm{H} & -0.72642 & 1.71443 & 0.21885\end{array}$

H $-0.83616 \quad 3.10713-1.82227$

H $\quad 0.81802 \quad 2.65549-2.28905$

H $-0.47235 \quad 1.41852-2.25594$

H -0.026794 .119780 .27679$

$\begin{array}{lllll}\mathrm{H} & 0.77892 & 3.17875 & 1.54928\end{array}$

$\begin{array}{lllll}\mathrm{H} & 1.67985 & 3.66278 & 0.08805\end{array}$

H $4.49839-0.61163-1.80158$

H $4.34002-0.40391-0.04131$

H $3.27328-1.54962-0.88905$

H $4.26067 \quad 1.86311-2.11858$

H $2.803992 .75293-1.63001$

H $3.97908 \quad 2.21859-0.40169$

$\begin{array}{llll}\text { H } & 1.18279 & 0.25482 & 3.78331\end{array}$

$\begin{array}{lllll}\mathrm{H} & 0.27515 & 1.42436 & 2.79394\end{array}$

H $0.06311-0.334262 .52676$

H $3.38741 \quad 1.234433 .18476$

$\begin{array}{llll}\text { H } & 3.97921 & 1.30588 & 1.51458\end{array}$

$\begin{array}{llll}\mathrm{H} & 2.76583 & 2.48333 & 2.08515\end{array}$

C $-4.00855 \quad 0.33366-1.11664$

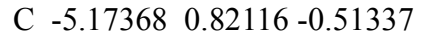

$\begin{array}{llll}\text { C } & -5.32178 & 0.77270 & 0.88460\end{array}$

$\begin{array}{llll}\text { C } & -4.29769 & 0.22710 & 1.67809\end{array}$

C $-3.13126-0.266881 .08077$

H $-3.887600 .37211-2.20406$

H $-5.97034 \quad 1.24417-1.13459$

H $-6.233801 .15729 \quad 1.35284$

H $-4.41245 \quad 0.181852 .76622$

H $-2.32990-0.705831 .68408$

Intermediate A3

SCF Energy $=-1158.54707664$

Enthalpy $0 \mathrm{~K}=-1157.881264$

Enthalpy $298 \mathrm{~K}=-1157.837872$

Free Energy 298K $=-1157.954499$

$\mathrm{SCF}(\mathrm{C} 6 \mathrm{H6})=-1158.55367146$

$\mathrm{SCF}(\mathrm{BP} 86-\mathrm{D} 3)=-0.14198470$

Lowest Frequencies $=15.322827 .0161 \mathrm{~cm}-1$

C $-2.10656-0.96072-0.91973$

C $-1.31882-0.62692-1.87689$

Rh $-0.11167-0.48801-0.22358$

$\begin{array}{lllll}\text { P } & -0.61582 & 1.75778 & 0.00784\end{array}$

C -2.526012 .026090 .15352$

C $-3.235542 .17864-1.20807$

P $2.37144-0.625230 .01355$ 
C $3.492610 .78151-0.70620$

$\begin{array}{llll}\text { C } & 3.77841 & 1.90126 & 0.31434\end{array}$

C $2.78108-2.20847-0.98162$

C $2.38137-2.01093-2.45844$

C $4.15404-2.88827-0.82602$

C $3.02141-0.846311 .80739$

C $4.53940-1.023812 .02296$

Cl $-0.16049-2.873140 .30364$

C $2.25393-1.940612 .57195$

C $4.815120 .37772-1.38951$

C $-0.14015 \quad 3.14442-1.24460$

C $-0.234342 .60304-2.68530$

C $0.11593 \quad 2.29846 \quad 1.69594$

$\begin{array}{llll}C & -0.30672 & 1.29285 & 2.78557\end{array}$

C $1.239973 .79611-1.05173$

$\begin{array}{llll}\text { C } & -0.03387 & 3.75549 & 2.18974\end{array}$

$\begin{array}{llll}\text { C } & -2.96963 & 3.17685 & 1.07579\end{array}$

H $2.02228-2.88519-0.54652$

$\begin{array}{lllll}\mathrm{H} & 2.73258 & 0.13203 & 2.24294\end{array}$

H $2.829631 .19024-1.49195$

H $-0.915913 .92138-1.09485$

H 1.186352 .119091 .49014

$\begin{array}{llll}\text { H } & -2.85850 & 1.07203 & 0.59864\end{array}$

H - $-1.09304-0.47199-2.92807$

C $-3.37217-1.39227-0.37880$

H $2.54263-1.914153 .63916$

H $1.16269-1.827592 .49417$

H $2.49264-2.944462 .18279$

H $4.77559-0.856313 .09030$

H $4.86601-2.046501 .77989$

H $5.15796-0.32849 \quad 1.43638$

H $2.42725-2.98273-2.98249$

H $1.34670-1.63534-2.53396$

H $3.04487-1.31327-2.99915$

H $4.17140-3.78540-1.47328$

H $5.00762-2.25922-1.12090$

H $4.32504-3.238010 .20392$

H $4.221572 .77362-0.19966$

$\begin{array}{llll}\mathrm{H} & 2.87557 & 2.25540 & 0.83376\end{array}$

H $4.49950 \quad 1.57664 \quad 1.08125$

H $5.275861 .27718-1.83977$

H $5.54561-0.03570-0.67484$

H $4.67397-0.35629-2.19558$

H $\quad 0.24958 \quad 1.486893 .72083$

$\mathrm{H}-1.38276 \quad 1.372103 .01770$

$\begin{array}{llll}\mathrm{H} & -0.10817 & 0.25183 & 2.47431\end{array}$

H 0.803573 .996662 .86966

H $-0.027104 .50247 \quad 1.38063$

H -0.962353 .900142 .76173$

H - $-0.011493 .41158-3.40573$

H $\quad 0.503351 .79548-2.83602$

H -1.22620 2.19711 -2.92991

H $1.337624 .64791-1.74965$

H $1.417454 .18124-0.03692$

H $2.047663 .08859-1.29384$

H $-4.327742 .16774-1.04241$

H -2.99099 $3.14091-1.69115$

H $-2.99637 \quad 1.35941-1.90091$

H -4.070773 .264261 .03252$

H -2.69997 3.009412 .12862

H -2.555994 .151520 .76272$

C $-4.52945-1.40838-1.20353$ 
C $-5.76689-1.80896-0.68608$

C $-5.88099-2.198990 .66029$

C $-4.74202-2.191241 .48428$

C $-3.49942-1.792290 .97531$

H $-4.44031-1.10508-2.25188$

H $-6.64839-1.81710-1.33660$

H $-6.85076-2.510751 .06220$

H $-4.82144-2.504992 .53080$

H -2.59909-1.81413 1.59605

Intermediate A4

SCF Energy $=-927.513883985$

Enthalpy $0 \mathrm{~K}=-926.926786$

Enthalpy $298 \mathrm{~K}=-926.888455$

Free Energy 298K = -926.992191

$\mathrm{SCF}(\mathrm{C} 6 \mathrm{H6})=-927.517548119$

$\mathrm{SCF}(\mathrm{BP} 86-\mathrm{D} 3)=-0.11836683$

Lowest Frequencies $=29.727531 .5434 \mathrm{~cm}-1$

C $3.18506-0.43514-1.70421$

P $2.422860 .07217-0.02053$

C $2.93581-1.205691 .29808$

C $3.25249 \quad 1.74740 \quad 0.41613$

Rh $-0.000020 .15432-0.00015$

$\begin{array}{llll}\text { P } & -2.42279 & 0.07217 & 0.02061\end{array}$

C $-2.93605-1.20541-1.29812$

$\begin{array}{lllll}\text { C } & 0.03991 & 2.13709 & 0.63831\end{array}$

C $-0.039862 .13699-0.63887$

Cl $-0.00008-2.24951-0.00030$

C $-3.18473-0.43532 \quad 1.70432$

$\begin{array}{llll}\text { C } & -3.25250 & 1.74745 & -0.41572\end{array}$

C $4.37972-1.73710 \quad 1.32201$

C $2.46227-0.715032 .68192$

H $2.26687-2.038951 .01177$

H $\quad 2.53103 \quad 2.15604 \quad 1.14888$

C $4.63722 \quad 1.70973 \quad 1.09085$

C $3.259792 .71067-0.78828$

H $2.71416 \quad 0.31037-2.37503$

C $4.71546-0.31892-1.86172$

C $2.70447-1.82968-2.14903$

H $-0.122622 .63901-1.59844$

$\begin{array}{lllll}\text { H } & 0.12274 & 2.63929 & 1.59778\end{array}$

C $-4.71514-0.31913 \quad 1.86196$

C $-2.70411-1.829932 .14891$

H -2.713770 .310112 .37519$

C $-4.37996-1.73684-1.32190$

C $-2.46274-0.71455-2.68198$

H $-2.26710-2.03878-1.01211$

C $-4.63735 \quad 1.70977-1.09021$

$\begin{array}{llll}\text { C } & -3.25961 & 2.71064 & 0.78873\end{array}$

H -2.53118 2.15616-1.14858

H $-4.954852 .74376-1.32335$

H $-4.631841 .14965-2.03780$

H $-5.41236 \quad 1.27089-0.44138$

$\begin{array}{llll}\mathrm{H} & -3.53931 & 3.72431 & 0.44756\end{array}$

H $-3.992462 .41174 \quad 1.55591$

H $-2.269932 .77923 \quad 1.26478$

H - $-2.55169-1.53402-3.41799$

H $-3.061130 .13355-3.05916$

H - $1.40417-0.40214-2.64687$

H - $4.47712-2.47268-2.14223$

H $-4.63922-2.26112-0.38853$

H -5.13324 -0.95247-1.49519 
H $-4.98768-0.567772 .90464$

H -5.101310 .691391 .65895$

H -5.25371 -1.02406 1.20973

H -3.02752 -2.01303 3.19013

H -3.14037-2.62663 1.52178

H - $1.61058-1.931872 .09024$

H $3.538963 .72443-0.44691$

H $3.993142 .41206-1.55510$

H $2.270332 .77893-1.26481$

H $4.954662 .74373 \quad 1.32403$

H 4.631591 .149632 .03844

$\begin{array}{lllll}\text { H } & 5.41233 & 1.27087 & 0.44212\end{array}$

H $2.55124-1.534573 .41786$

H $3.06053 \quad 0.133103 .05926$

H $1.40367-0.402742 .64671$

H $4.47680-2.473002 .14230$

H $4.63913-2.261270 .38863$

H $5.13288-0.952661 .49551$

H $3.02810-2.01268-3.19021$

H $3.14057-2.62647-1.52191$

H $1.61093-1.93158-2.09063$

H $4.98807-0.56729-2.90444$

H $5.101640 .69155-1.65844$

H $5.25405-1.02398-1.20965$

Intermediate A5

SCF Energy $=-927.485527451$

Enthalpy $0 \mathrm{~K}=-926.899295$

Enthalpy $298 \mathrm{~K}=-926.860704$

Free Energy 298K = -926.965746

$\mathrm{SCF}(\mathrm{C} 6 \mathrm{H6})=-927.492075525$

$\mathrm{SCF}(\mathrm{BP} 86-\mathrm{D} 3)=-0.12373672$

Lowest Frequencies $=17.942631 .4878 \mathrm{~cm}-1$

C $3.10819-0.85318-0.87506$

$\begin{array}{llll}\text { P } & 1.83280 & 0.30224 & -0.03002\end{array}$

Rh $-0.17969-1.13859-0.00850$

Cl $1.23240-2.923460 .89338$

$\begin{array}{llll}\text { C } & 2.52137 & 0.83167 & 1.68707\end{array}$

C $1.85333 \quad 1.96344-1.03270$

C $-1.39924-2.85066-0.35219$

C $-1.09827-2.31065-1.46590$

P $-1.961780 .32512-0.01214$

$\begin{array}{lll}\text { C }-2.46891 & 1.48782 & -1.46487\end{array}$

C $-1.73682 \quad 1.45361 \quad 1.52156$

C $-3.61827-0.635140 .29429$

C $4.61395-0.54494-0.78368$

H $2.91957-1.77231-0.28993$

C $2.65208-1.15869-2.31668$

C $2.58909-0.338502 .68516$

$\begin{array}{llll}\text { C } & 3.84168 & 1.62882 & 1.72547\end{array}$

H $1.71011 \quad 1.509632 .02066$

C $3.146212 .33215-1.78898$

H $\quad 1.07721 \quad 1.76418-1.79565$

C $1.394543 .17304-0.19308$

H $-3.56029 \quad 1.63042-1.33722$

C $-1.79728 \quad 2.87041-1.49493$

C $-2.201840 .77490-2.80571$

H $\quad-0.78274 \quad 1.93995 \quad 1.24835$

C $-2.76272 \quad 2.568021 .83629$

C $-1.45850 \quad 0.585762 .76598$

H $-3.26199-1.501210 .88108$

C $-4.680120 .11648 \quad 1.11910$ 
C $-4.27862-1.17528-0.98967$

H $-1.12068-2.22690-2.54729$

$\begin{array}{llll}\mathrm{H} & -1.75779 & -3.65098 & 0.28248\end{array}$

$\begin{array}{lllll}\mathrm{H} & 2.83112 & 0.05385 & 3.69067\end{array}$

H $1.64848-0.905352 .74201$

H $3.37488-1.060132 .40717$

H $3.978602 .05240 \quad 2.73778$

H $4.709920 .98323 \quad 1.52467$

H 3.875672 .464591 .01140

H $3.23629-2.00872-2.71310$

H $1.58564-1.44269-2.33419$

H $2.79689-0.30953-3.00774$

H $5.16355-1.32914-1.33757$

H $4.904420 .42386-1.21781$

H $4.97588-0.57805 \quad 0.25579$

H $1.205264 .03806-0.85467$

$\begin{array}{lllll}\mathrm{H} & 0.46631 & 2.98218 & 0.36664\end{array}$

H $2.164693 .48293 \quad 0.53145$

$\mathrm{H} \quad 2.968303 .25130-2.37874$

H $3.986942 .54234-1.10775$

H $3.466201 .54963-2.49182$

H $-1.11404 \quad 1.219393 .60346$

H $-2.36705 \quad 0.058203 .10411$

H $-0.68649-0.178152 .56561$

H $-2.25610 \quad 3.388812 .37576$

H $-3.23723 \quad 3.005240 .94407$

H -3.569522 .206352 .49099$

H $-2.543791 .40774-3.64522$

H $-1.119140 .59549-2.92744$

H -2.71215 - $0.19586-2.88557$

H $-2.243743 .47296-2.30722$

H $-1.907403 .44311-0.56244$

H $-0.722742 .78033-1.71430$

H -5.13016-1.82134-0.70686

H $-4.68823-0.36227-1.61379$

H $-3.58935-1.78141-1.59315$

H $-5.59105-0.506971 .18450$

H $-4.35590 \quad 0.324462 .14906$

H $-4.97738 \quad 1.070850 .65032$

Intermediate A6

SCF Energy $=-1245.46690196$

Enthalpy $0 \mathrm{~K}=-1244.660591$

Enthalpy $298 \mathrm{~K}=-1244.610731$

Free Energy 298K = -1244.737786

$\mathrm{SCF}(\mathrm{C} 6 \mathrm{H} 6)=-1245.46935937$

$\mathrm{SCF}(\mathrm{BP} 86-\mathrm{D} 3)=-0.17810461$

Lowest Frequencies $=17.932230 .6585 \mathrm{~cm}-1$

C $3.31876 \quad 0.61980-1.11100$

P $2.29470-0.62663-0.06852$

Rh $-0.08370-0.249970 .02405$

$\mathrm{Cl}-0.24526-2.44058 \quad 0.98574$

C $2.46812-2.32285-0.93611$

C $3.22625-0.69251 \quad 1.61093$

P $-2.54853-0.66977-0.06930$

$\begin{array}{llll}\text { C } & -3.97695 & 0.56196 & -0.54738\end{array}$

$\begin{array}{lllll}\text { B } & 0.06400 & 1.75334 & 0.70274\end{array}$

$\begin{array}{llll}\text { C } & 0.14238 & 2.54918 & 2.09911\end{array}$

$\begin{array}{lllll}\mathrm{N} & 0.02331 & 1.83590 & -0.62633\end{array}$

C $-0.068172 .65380-1.83572$

C $-2.92626-2.00666-1.42875$

C $-3.38854-1.193041 .61500$ 
C $3.79899-3.09204-0.85052$

H $1.71314-2.89965-0.37330$

C $1.95349-2.20796-2.38548$

C $2.61618-1.704432 .59726$

C $4.76075-0.850281 .57060$

H $3.00314 \quad 0.32256 \quad 1.99433$

C $3.63411 \quad 1.89104-0.30185$

C $4.583780 .11223-1.83252$

H $2.578450 .89409-1.88511$

H $-4.34045-0.63096 \quad 1.58436$

C $-3.75629-2.67198 \quad 1.81617$

C $-2.58038-0.670302 .81400$

H $-4.01603-1.89907-1.59893$

C $-2.16983-1.61430-2.71519$

C $-2.62558-3.49033-1.14627$

H $-4.87888-0.04111-0.31704$

$\begin{array}{llll}\text { C } & -3.97150 & 1.78804 & 0.38240\end{array}$

C $-4.095180 .99121-2.01851$

C 1.197153 .684512 .01272

C $-1.23130 \quad 3.21970 \quad 2.37269$

C $0.49621 \quad 1.64097 \quad 3.29958$

C $-1.261643 .63577-1.71369$

C $1.208843 .51471-2.02532$

C $-0.24152 \quad 1.76258-3.08173$

H $1.18144 \quad 4.284462 .94364$

H 2.220993 .291741 .89626

H $0.997394 .37127 \quad 1.17186$

H - $1.182193 .80473 \quad 3.31183$

$\mathrm{H}-1.518383 .91270 \quad 1.56264$

H -2.04004 2.480032 .48491

H $\quad 0.47234 \quad 2.22274 \quad 4.24169$

$\begin{array}{llll}\text { H } & -0.20594 & 0.79832 & 3.39618\end{array}$

H $1.508691 .21467 \quad 3.20006$

$\mathrm{H}-1.349144 .26103-2.62023$

H $-2.210573 .09868-1.57424$

H - $1.117974 .30702-0.84958$

H $1.078514 .19871-2.88287$

H $1.410484 .12469-1.12944$

H $2.091202 .88931-2.22574$

H $-0.283992 .37862-3.99680$

H $0.601701 .05727-3.17256$

H - $1.164191 .16727-3.01632$

H $4.080042 .65700-0.96229$

H $2.724612 .32305 \quad 0.13819$

H $4.35927 \quad 1.701100 .50664$

H $\quad 5.002250 .93811-2.43831$

H $5.37373-0.20653-1.13385$

H $4.38228-0.72305-2.51946$

H $3.11384-1.597423 .57908$

H $1.53506-1.559462 .72757$

H $2.76771-2.743632 .25855$

H $5.15623-0.733752 .59683$

H $5.06614-1.846961 .21771$

H $5.26168-0.09996 \quad 0.94169$

H $1.79714-3.21695-2.80822$

H $\quad 0.99018-1.66996-2.42181$

H $2.65873-1.67851-3.04878$

H $3.69486-4.03995-1.41173$

H $4.65367-2.54953-1.28386$

H $4.05134-3.361750 .18704$

H -2.46864 -2.28711 -3.54062

H - $2.34797-0.58126-3.04332$ 
H $-1.08443-1.72900-2.55926$

H $-2.89199-4.07090-2.05005$

H $-1.55748-3.64830-0.93566$

H $-3.19700-3.90361-0.30669$

H $-3.16092-0.818163 .74409$

H $-1.62400-1.208282 .90352$

$\begin{array}{llll}\mathrm{H} & -2.35578 & 0.40435 & 2.72298\end{array}$

H $-4.25487-2.778702 .79821$

H $-4.46287-3.03693 \quad 1.05320$

H -2.86296 -3.314901 .81852$

H $-4.83942 \quad 2.436750 .16245$

$\mathrm{H}-4.03176 \quad 1.510771 .44764$

$\begin{array}{lllll}\mathrm{H} & -3.05828 & 2.38664 & 0.24578\end{array}$

H $-4.92010 \quad 1.72118-2.11821$

H -3.18208 $1.48185-2.39092$

H $-4.328760 .14793-2.68659$

Intermediate A7

SCF Energy $=-1245.46856107$

Enthalpy $0 \mathrm{~K}=-1244.662337$

Enthalpy $298 \mathrm{~K}=-1244.612498$

Free Energy 298K = -1244.739007

$\mathrm{SCF}(\mathrm{C} 6 \mathrm{H6})=-1245.47187563$

$\mathrm{SCF}(\mathrm{BP} 86-\mathrm{D} 3)=-0.18034927$

Lowest Frequencies $=26.995334 .3827 \mathrm{~cm}-1$

C -2.82444 - 1.612261 .45742

P $-2.34093-0.52179-0.04381$

Rh $0.14469-0.36650-0.11232$

Cl $-0.07842-2.82858-0.07041$

C $-2.69942-1.58732-1.61051$

C $-3.636660 .90259-0.15810$

B $2.10661-1.242650 .46396$

C 2.82736-1.98131 1.68157

N $2.14318-0.78063-0.77804$

C $2.58899-1.04423-2.15499$

$\begin{array}{lllll}\text { P } & 0.61104 & 1.89044 & 0.14552\end{array}$

$\begin{array}{llll}\text { C } & 0.70111 & 2.60600 & 1.93501\end{array}$

C $-0.69195 \quad 3.03005-0.68809$

C $2.30507 \quad 2.47335-0.58405$

C $-4.31630-1.824981 .77582$

H $-2.38078-2.575791 .14779$

C $-2.05540-1.145202 .70884$

H $-1.79561-2.21926-1.61170$

C $-3.90017-2.55090-1.57188$

C $-2.66555-0.73722-2.89431$

C $-5.05603 \quad 0.54430-0.64762$

C $-3.74466 \quad 1.700551 .15350$

H $-3.18355 \quad 1.55933-0.92544$

$\begin{array}{lllll}\mathrm{H} & 1.25312 & 3.55951 & 1.81284\end{array}$

$\begin{array}{llll}\text { C } & -0.65594 & 2.90169 & 2.59412\end{array}$

$\begin{array}{llll}\text { C } & 1.48460 & 1.68157 & 2.88343\end{array}$

H $-1.60252 \quad 2.61312-0.22495$

C $-0.691524 .55338-0.41974$

C $-0.77044 \quad 2.73036-2.19606$

H $2.36024 \quad 1.86614-1.50355$

C $2.407163 .95985-0.97264$

$\begin{array}{llll}\text { C } & 3.52212 & 2.10167 & 0.28208\end{array}$

C $2.52319-2.56173-2.45938$

C $1.70241-0.30708-3.18103$

C $4.05450-0.56159-2.28917$

C $1.90006-2.245402 .88935$

C $3.32556-3.353731 .13991$ 
C $4.06991-1.179772 .15101$

H $4.62177-1.761152 .91457$

H $4.76740-0.976301 .31972$

H $3.79516-0.216012 .61164$

H $3.82708-3.910451 .95561$

H $2.48911-3.963330 .76284$

H $4.05796-3.22810 \quad 0.32336$

H $2.44789-2.807643 .67032$

H $1.54261-1.305943 .34382$

H $1.01997-2.834652 .58605$

H $4.44365-0.79293-3.29650$

H $4.135870 .52614-2.12840$

H $4.69808-1.06758-1.54969$

H $2.83422-2.75417-3.50220$

H $3.19476-3.12322-1.78992$

H $1.50098-2.94064-2.30550$

H $2.05584-0.52351-4.20431$

H $\quad 0.65660-0.64349-3.08908$

H $1.725850 .78552-3.03621$

H $-2.18692-1.879903 .52465$

H $-0.97758-1.054502 .49195$

H $-2.42198-0.17148 \quad 3.07979$

H $-4.40385-2.58145 \quad 2.57784$

H $-4.79988-0.907332 .15143$

H $-4.89815-2.18990 \quad 0.91634$

H -3.90646 -3.14202 -2.50683

H $-3.81453-3.26933-0.74112$

H $-4.87691-2.04725-1.49878$

H -2.70431 -1.40405 -3.77499

H -3.51266 - $0.03551-2.98166$

H - $1.73220-0.15410-2.96488$

$\begin{array}{llll}\mathrm{H} & -4.30096 & 2.64020 & 0.98088\end{array}$

H -4.297131 .133361 .92085$

H -2.76566 1.959761 .57675

H $-5.664901 .46768-0.67809$

H $-5.07026 \quad 0.11647-1.66012$

H $-5.56498-0.157470 .03108$

H - $1.714264 .94789-0.56312$

$\begin{array}{llll}\mathrm{H} & -0.37561 & 4.82430 & 0.59994\end{array}$

H - $0.039025 .09649-1.11942$

H - $1.672173 .19260-2.63863$

H $\quad 0.102203 .13404-2.73849$

H $-0.80636 \quad 1.64439-2.38246$

H $1.57734 \quad 2.15709 \quad 3.87771$

$\begin{array}{lllll}\mathrm{H} & 0.94358 & 0.72943 & 3.00924\end{array}$

$\begin{array}{llll}\text { H } & 2.49544 & 1.44618 & 2.52822\end{array}$

$\begin{array}{llll}\text { H } & -0.48753 & 3.39487 & 3.56937\end{array}$

H - 1.313113 .558252 .00566

H $-1.19073 \quad 1.959092 .80079$

H $4.446322 .28819-0.29607$

H $3.582792 .72668 \quad 1.18965$

H $3.519261 .04106 \quad 0.56660$

H $3.430964 .16494-1.33743$

H $1.714624 .24378-1.77813$

H $2.233604 .63228-0.11443$

Intermediate B1

SCF Energy $=-1191.70884814$

Enthalpy 0K $=-1191.074348$

Enthalpy $298 \mathrm{~K}=-1191.032196$

Free Energy 298K = -1191.147001

SCF $($ C6H6) $=-1191.71334002$ 
$\mathrm{SCF}(\mathrm{BP} 86-\mathrm{D} 3)=-0.1311964$

Lowest Frequencies $=19.495424 .5678 \mathrm{~cm}-1$

C $-0.46428 \quad 5.09880 \quad 1.63279$

C $-0.54177 \quad 3.71650 \quad 1.83129$

C -0.284902 .819300 .75876$

C $0.04482 \quad 3.35654-0.51006$

C $\quad 0.12318 \quad 4.74312-0.69914$

C $-0.13030 \quad 5.619590 .36862$

C $-0.35893 \quad 1.39622 \quad 0.98574$

$\begin{array}{llll}\text { C } & -0.49877 & 0.36335 & 1.73544\end{array}$

$\mathrm{Rh}-0.28073-0.32652-0.22408$

P $2.08616-0.57971-0.05273$

C $2.84856-0.50727-1.79817$

C $4.29314-0.99837-2.00641$

Cl $-0.29093-1.56665-2.25676$

$\mathrm{N}-2.47941-0.31026-0.36630$

C $-3.39970 \quad 0.28622-1.32925$

C $-4.554360 .94311-0.53314$

B $-2.20498-1.183360 .56676$

C $-2.54960-2.32473 \quad 1.61316$

C $-3.42046-3.369490 .85861$

C $-1.30326-3.040062 .18286$

C $-3.38056-1.722782 .77749$

$\begin{array}{lrrr}\text { C } & 2.94120 & 0.74041 & 1.04589\end{array}$

C $4.42532 \quad 1.03843 \quad 0.75632$

C $2.51259-2.286920 .69457$

C $3.96921-2.52617 \quad 1.14082$

$\begin{array}{llll}\text { C } & 2.72873 & 0.47482 & 2.55067\end{array}$

C $-2.68363 \quad 1.35658-2.17866$

C $-3.95828-0.82038-2.25800$

C $2.03241-3.42246-0.23040$

C $2.605340 .88237-2.41777$

H $2.35739 \quad 1.64395 \quad 0.78790$

H $1.86882-2.28753 \quad 1.59529$

H $2.16841-1.20526-2.32222$

H $-0.62967-0.085312 .71436$

H $\quad 2.10098-4.38658 \quad 0.30539$

H $0.99531-3.27465-0.56961$

H $2.66421-3.50110-1.13218$

H $4.03400-3.514101 .63295$

H $4.66748-2.54105 \quad 0.28960$

H $4.33116-1.777871 .86133$

H $2.93067 \quad 0.87793-3.47359$

H $1.531731 .13305-2.40395$

H $3.159681 .68599-1.90279$

H $4.52788-0.95236-3.08623$

H $5.04664-0.38893-1.48350$

H $4.42379-2.04650-1.69485$

H $3.04623 \quad 1.363883 .12504$

H $1.67284 \quad 0.28200 \quad 2.78898$

H $3.32793-0.377112 .91150$

H $4.76731 \quad 1.84636 \quad 1.42962$

H $5.07273 \quad 0.165240 .93924$

H $4.595341 .38116-0.27563$

H $-5.282351 .40607-1.22271$

H $-5.08690 \quad 0.191510 .07453$

H $-4.16570 \quad 1.723590 .14183$

H -4.66000 - $0.38663-2.99281$

H -3.13259-1.31301-2.79468

H $-4.49940-1.58271-1.67200$

H $-3.39265 \quad 1.80065-2.89890$

H $-2.286782 .16296-1.54024$ 
H $-1.849870 .90247-2.73797$

H $-3.70949-4.18587 \quad 1.54827$

H $-4.34712-2.919050 .46412$

H -2.87404 -3.81587 0.01079

H $-3.68830-2.522993 .47788$

H $-2.80427-0.978303 .35427$

H $-4.29396-1.224732 .41025$

$\mathrm{H}-1.61215-3.863942 .85410$

H $-0.68100-3.46710 \quad 1.37907$

H $-0.66790-2.354892 .76993$

H $\quad 0.23212 \quad 2.66638-1.33777$

H $0.378615 .14086-1.68700$

$\begin{array}{llll}\mathrm{H} & -0.07225 & 6.70247 & 0.21832\end{array}$

H $-0.66758 \quad 5.776922 .46854$

H $-0.80467 \quad 3.310102 .81318$

Intermediate B2

SCF Energy $=-1191.70554937$

Enthalpy $0 \mathrm{~K}=-1191.071467$

Enthalpy $298 \mathrm{~K}=-1191.029147$

Free Energy 298K = -1191.145422

SCF $($ C6H6) $=-1191.70965258$

SCF $(B P 86-D 3)=-0.13197742$

Lowest Frequencies $=8.902820 .5497 \mathrm{~cm}-1$

C $-0.59876 \quad 1.18459-1.19846$

$\mathrm{Rh} 0.00386-0.63036-0.23075$

$\mathrm{N}-2.05623-1.36818-0.52961$

C $-2.55706-2.39394-1.43925$

C $-2.25973-3.79588-0.85433$

$\begin{array}{llll}\text { C } & -0.18715 & 0.32232 & -2.05035\end{array}$

B $-2.14155 \quad-0.75587 \quad 0.61849$

$\begin{array}{llll}\text { C } & -2.84132 & -0.27514 & 1.95161\end{array}$

$\begin{array}{llll}\text { C } & -3.92450 & 0.78099 & 1.60192\end{array}$

$\begin{array}{llll}\text { P } & 2.29710 & -0.01025 & 0.01900\end{array}$

C $2.697340 .93597 \quad 1.63194$

C $4.09516 \quad 1.57892 \quad 1.73677$

Cl $0.27034-2.343451 .40646$

C -3.52819-1.53605 2.55296

$\begin{array}{llll}\text { C } & -1.86889 & 0.30301 & 3.00298\end{array}$

C $3.27776-1.64450 \quad 0.02537$

C $4.75500-1.642690 .45900$

C $2.952741 .07362-1.42295$

C $4.453620 .97570-1.75974$

C $3.06134-2.36712-1.32009$

C $2.518642 .54572-1.27919$

$\begin{array}{llll}\text { C } & 2.40020 & 0.07645 & 2.87522\end{array}$

C $-4.08640-2.19123-1.57181$

C $-1.90882-2.27412-2.83414$

H $2.385780 .65274-2.27533$

H $1.94833 \quad 1.75071 \quad 1.59143$

H $2.69686-2.203110 .78310$

C $-1.181232 .44994-0.82101$

$\begin{array}{lllll}\mathrm{H} & 0.05746 & 0.01741 & -3.06258\end{array}$

$\begin{array}{llll}\mathrm{H} & 2.45265 & 0.71007 & 3.77920\end{array}$

H $1.40908-0.398032 .82800$

H $3.14496-0.729582 .99422$

H 4.156192 .147712 .68289

H $4.897280 .82516 \quad 1.75846$

H 4.311512 .281620 .91859

H $3.45646-3.39685-1.25678$

H $1.98660-2.43527-1.56410$

H $3.57590-1.86643-2.15882$ 
H $\quad 5.13596-2.680270 .41902$

H $5.40345-1.03260-0.18865$

H $4.88342-1.297001 .49695$

H $2.721413 .08034-2.22498$

H $1.445542 .64847-1.06407$

H $3.079393 .06740-0.48620$

H $4.667751 .62163-2.63159$

H $5.096921 .32102-0.93410$

H $4.76259-0.04620-2.02791$

H $-4.51950-2.95641-2.23967$

H $-4.57774-2.27767-0.58800$

H -4.31189-1.19434-1.98618

H -2.61806 -4.58257-1.54206

H - $1.17838-3.92529-0.69078$

H $-2.76179-3.924230 .11866$

H $-2.31780-3.04910-3.50472$

H -2.11046-1.28557-3.27846

H $-0.81697-2.41297-2.76638$

H -4.06188 -1.25929 3.48278

H $-4.26840-1.971701 .85952$

H $-2.78743-2.315032 .79930$

H -4.484091 .058802 .51592$

H $-3.47713 \quad 1.699401 .18689$

$\begin{array}{llll}\mathrm{H} & -4.65217 & 0.39327 & 0.86827\end{array}$

H $-2.42478 \quad 0.56462 \quad 3.92364$

H - $1.08576-0.427263 .26250$

H $-1.37695 \quad 1.217512 .63464$

$\begin{array}{llll}\text { C } & -0.95584 & 3.02986 & 0.45229\end{array}$

$\begin{array}{llll}C & -1.51210 & 4.27392 & 0.78062\end{array}$

C $-2.308724 .96224-0.14932$

C $-2.542164 .39696-1.41652$

C $-1.98758 \quad 3.15737-1.75344$

H $-0.338002 .48601 \quad 1.17359$

H $-1.32586 \quad 4.70582 \quad 1.76969$

H -2.747645 .930830 .11074$

H -3.16483 $4.92580-2.14612$

H -2.17256 2.71436-2.73706

Intermediate B3

SCF Energy $=-1104.78122853$

Enthalpy $0 \mathrm{~K}=-1104.286457$

Enthalpy $298 \mathrm{~K}=-1104.251129$

Free Energy 298K = -1104.353731

$\mathrm{SCF}(\mathrm{C} 6 \mathrm{H} 6)=-1104.78914311$

SCF $($ BP86-D3) $=-0.09812302$

Lowest Frequencies $=13.586621 .2033 \mathrm{~cm}-1$

C $0.97540-1.78578-2.20614$

C $1.84442-1.63739-1.31395$

$\mathrm{Rh}-0.24915-0.73990-0.76280$

Cl $-0.62111-2.81573 \quad 0.34498$

$\begin{array}{llll}\text { P } & -2.23617 & 0.17215 & 0.19067\end{array}$

$\begin{array}{llll}\text { C } & -2.16059 & 0.55421 & 2.06130\end{array}$

C -1.48936 -0.559192 .88664$

$\begin{array}{llll}C & -2.72941 & 1.86267 & -0.60227\end{array}$

C $-2.016713 .04808 \quad 0.07682$

C $-4.235042 .15171-0.75806$

C $-3.79170-0.88868-0.12559$

C $-3.96662-2.110220 .79376$

C $-0.113290 .77166-2.19800$

C $0.596871 .15410-1.20319$

C $-3.80679-1.31289-1.60825$

C $-3.50936 \quad 0.979542 .67432$ 
C $2.97986-1.59123-0.43943$

H $\quad 0.57915-2.17708-3.13269$

H -2.29536 $1.76208-1.61376$

H $-4.63267-0.195640 .05904$

H $-1.482681 .42826 \quad 2.07508$

H $-0.48906 \quad 0.86596-3.21138$

C $1.481302 .04604-0.49176$

H $-4.89585-2.638130 .50866$

H -3.12136 -2.80866 0.69771

$\mathrm{H}-4.06216-1.828721 .85463$

H $-4.75204-1.83728-1.83912$

H $-3.72137-0.45592-2.29875$

H -2.97001-2.00035 -1.81867

H $-1.33843-0.198853 .92118$

H -2.10397-1.47202 2.93210

H $-0.51459-0.851512 .46753$

H $-3.36277 \quad 1.24612 \quad 3.73698$

H $-3.94900 \quad 1.856312 .17304$

H $-4.24996 \quad 0.163182 .64221$

H $-4.362043 .13559-1.24622$

H $-4.75167 \quad 1.40927-1.38683$

$\begin{array}{llll}\mathrm{H} & -4.76121 & 2.20213 & 0.21035\end{array}$

H - $-2.137323 .95370-0.54471$

H -2.450003 .274391 .06639$

$\begin{array}{llll}\mathrm{H} & -0.93645 & 2.87751 & 0.20367\end{array}$

C $4.12356-0.83296-0.79845$

C $5.24831-0.82103 \quad 0.03579$

C $5.25016-1.55588 \quad 1.23353$

C $4.11755-2.306731 .59655$

C $2.98622-2.329410 .77265$

H $4.11341-0.26210-1.73152$

H $6.12709-0.23378-0.25027$

H $6.13199-1.543861 .88272$

H $4.11715-2.881912 .52855$

H $2.09363-2.903201 .03974$

C $1.952173 .23183-1.11761$

C $2.80247 \quad 4.10474-0.43042$

C 3.199123 .820120 .88946

C $2.741242 .64946 \quad 1.51656$

C $1.89203 \quad 1.76737 \quad 0.83516$

H $1.64105 \quad 3.45243-2.14384$

H $3.158625 .01438-0.92595$

$\begin{array}{llll}\text { H } & 3.86425 & 4.50697 & 1.42302\end{array}$

H 3.052772 .417762 .54049

H $1.54302 \quad 0.84270 \quad 1.30547$

Intermediate B4

SCF Energy $=-873.719756660$

Enthalpy 0K $=-873.304006$

Enthalpy $298 \mathrm{~K}=-873.273733$

Free Energy $298 \mathrm{~K}=-873.362889$

$\mathrm{SCF}(\mathrm{C} 6 \mathrm{H} 6)=-873.72572991$

$\mathrm{SCF}(\mathrm{BP} 86-\mathrm{D} 3)=-0.08249545$

Lowest Frequencies $=22.039631 .4326 \mathrm{~cm}-1$

$\begin{array}{llll}\text { C } & 2.97878 & 0.05571 & 1.07528\end{array}$

$\begin{array}{llll}\text { C } & 2.85030 & 0.24234 & -0.32368\end{array}$

C $3.910010 .87082-1.03099$

C $5.05801 \quad 1.29336-0.35214$

C $5.175121 .10251 \quad 1.03729$

C $4.132530 .48301 \quad 1.74618$

C $1.67027-0.20577-1.02135$

C $0.91502-0.43715-2.02881$ 
Rh $-0.02467-1.26263-0.33576$

P $-1.380050 .69972-0.03446$

$\begin{array}{llll}\text { C } & -3.24022 & 0.38607 & -0.32446\end{array}$

C $-3.40283-0.43371-1.62046$

C $0.67945-3.09769-1.30128$

C $1.24427-3.05146-0.18519$

H $1.79983-3.322670 .69893$

$\mathrm{Cl}-1.29426-2.43586 \quad 1.29597$

C $-1.19542 \quad 1.618351 .62957$

C -2.192742 .778341 .81717$

C $-0.984392 .05618-1.34811$

C -2.15706 2.92203-1.84844

C -1.173690 .686972 .85516$

$\begin{array}{llll}\text { C } & 0.20201 & 2.94227 & -0.92171\end{array}$

$\begin{array}{llll}\text { C } & -3.98697 & -0.28447 & 0.84221\end{array}$

H $\quad 0.37717-3.41487-2.28751$

H $-0.636961 .43620-2.19463$

H $-3.66822 \quad 1.39386-0.47799$

$\begin{array}{llll}\mathrm{H} & -0.18117 & 2.04812 & 1.52731\end{array}$

H $0.69429-0.42533-3.09075$

H $-5.04036-0.443710 .54525$

H -3.54120 - 1.259001 .09490

H $-3.99347 \quad 0.33826 \quad 1.75127$

H $-4.47517-0.54087-1.86581$

H $-2.90556 \quad 0.03361-2.48812$

H -2.97669-1.44309-1.49132

H $-0.89303 \quad 1.275443 .74819$

$\begin{array}{llll}\mathrm{H} & -2.15681 & 0.22987 & 3.04702\end{array}$

H - $0.45652-0.138322 .73444$

H -1.972073 .305082 .76358$

H -2.138293 .521681 .00606$

H -3.233542 .419111 .87997$

H -1.78369 $3.61716-2.62299$

H $-2.962842 .32886-2.30865$

H -2.60252 $3.53957-1.05036$

H $0.53744 \quad 3.54657-1.78394$

H $-0.08042 \quad 3.64852-0.12185$

H $1.065942 .35410-0.57471$

H $3.81851 \quad 1.01543-2.11220$

H $5.869471 .77369-0.90924$

H 6.076661 .434291 .56250

H $4.21937 \quad 0.32905 \quad 2.82698$

H $2.15850-0.434751 .61085$

\section{TS(B1-C1)}

SCF Energy $=-1191.70757405$

Enthalpy 0K $=-1191.074041$

Enthalpy $298 \mathrm{~K}=-1191.032556$

Free Energy 298K = -1191.145837

$\mathrm{SCF}(\mathrm{C} 6 \mathrm{H} 6)=-1191.71211263$

$\mathrm{SCF}(\mathrm{BP} 86-\mathrm{D} 3)=-0.13146327$

Lowest Frequencies = $-94.991913 .6886 \mathrm{~cm}-1$

C $-0.168724 .99158 \quad 1.80178$

C $-0.24678 \quad 3.60329 \quad 1.95589$

C $-0.27382 \quad 2.75500 \quad 0.81596$

C $-0.22776 \quad 3.34167-0.47284$

C $-0.15370 \quad 4.73362-0.61660$

$\begin{array}{llll}C & -0.12034 & 5.56299 & 0.51675\end{array}$

$\begin{array}{llll}C & -0.35896 & 1.32441 & 0.98883\end{array}$

$\begin{array}{llll}\text { C } & -0.59463 & 0.26748 & 1.69985\end{array}$

Rh $-0.28068-0.26989-0.31119$

P $2.07417-0.60799-0.08141$ 
C $2.98913-0.02051-1.64613$

C $4.41964-0.52703-1.90544$

$\mathrm{Cl}-0.13755-0.81738-2.62588$

N $-2.43873-0.34337-0.37636$

C $-3.481620 .06611-1.31439$

$\begin{array}{llll}\text { C } & -4.76263 & 0.36321 & -0.49652\end{array}$

B $-2.10217-1.068330 .68002$

C $-2.47761-2.21237 \quad 1.73043$

C $-3.40899-3.210850 .98673$

C $-1.24455-2.992902 .24191$

C $-3.25291-1.617532 .93519$

C $2.84663 \quad 0.26562 \quad 1.43909$

$\begin{array}{llll}\text { C } & 4.35500 & 0.57727 & 1.38731\end{array}$

C $2.41796-2.47780 \quad 0.14736$

C $3.84277-2.89475 \quad 0.56733$

C $2.49284-0.460852 .75285$

C $-3.05134 \quad 1.34311-2.06792$

C $-3.75643-1.07197-2.32889$

C $1.95497-3.28519-1.08121$

C $2.884891 .51143-1.77654$

H $2.29827 \quad 1.22643 \quad 1.43502$

H $1.73288-2.713260 .98475$

H $2.32340-0.44981-2.41852$

H $-0.67065-0.13907 \quad 2.70340$

H $2.00542-4.36482-0.85019$

H $\quad 0.92705-3.03527-1.38461$

H $2.60577-3.10366-1.95388$

H $3.85167-3.982620 .76487$

H $4.58361-2.70260-0.22402$

H $4.18995-2.394641 .48401$

H $3.21747 \quad 1.81700-2.78482$

H $1.847441 .85770-1.64841$

H $3.515802 .04637-1.04591$

H $4.77557-0.10400-2.86336$

H $5.13918-0.22450-1.12823$

H $4.46053-1.62286-2.00499$

H $2.70842 \quad 0.20070 \quad 3.61130$

H $1.43008-0.740182 .80104$

H $3.09022-1.377342 .88953$

H 4.641601 .126172 .30372

H $4.97077-0.335951 .34852$

H 4.628921 .209690 .52971

H $-5.579690 .68291-1.16693$

H $-5.10067-0.535430 .04769$

H $-4.57788 \quad 1.165740 .23719$

H $-4.54178-0.76532-3.04282$

H $-2.83780-1.31067-2.88621$

H $-4.09936-1.98175-1.80779$

H $-3.848041 .66067-2.76300$

H -2.86019 2.16547 -1.35807

H -2.13372 $1.15033-2.64598$

H -3.70990 -4.02816 1.67030

H $-4.32911-2.720230 .62676$

H $-2.90586-3.66406 \quad 0.11573$

H $-3.57728-2.426773 .61739$

H $-2.63521-0.916423 .52321$

H $-4.15366-1.070592 .60823$

H - $1.56220-3.806462 .92171$

H $-0.68336-3.448191 .40777$

H $-0.54501-2.349362 .80281$

H $-0.271672 .68672-1.34865$

H $-0.125025 .17282-1.61925$ 
$\begin{array}{llll}\mathrm{H} & -0.06023 & 6.65010 & 0.40168\end{array}$

$\mathrm{H}-0.147325 .633732 .68875$

H - 0.288023 .156822 .95473

\section{TS(B1-C3)}

SCF Energy $=-1191.66845888$

Enthalpy $0 \mathrm{~K}=-1191.034797$

Enthalpy $298 \mathrm{~K}=-1190.993705$

Free Energy 298K = -1191.104911

SCF $($ C6H6) $=-1191.67381137$

$\mathrm{SCF}(\mathrm{BP} 86-\mathrm{D} 3)=-0.13315836$

Lowest Frequencies $=-339.825322 .6319 \mathrm{~cm}-1$

C $5.21918-1.984641 .47481$

C $4.08229-1.164851 .48459$

C $2.93903-1.515860 .72334$

C $2.97261-2.69899-0.05886$

C $4.12011-3.50034-0.08087$

C $5.24463-3.147690 .68695$

C $1.72155-0.739960 .84126$

C $0.53051-0.676891 .38559$

Rh $-0.189210 .09826-0.32402$

P $-2.42900-0.550890 .09052$

C $-2.91207-1.80991-1.25663$

C $-4.39652-2.16928-1.44420$

$\mathrm{Cl}-0.71323 \quad 0.43009-2.67870$

$\begin{array}{lllll}\mathrm{N} & 1.77835 & 0.99750 & -0.30255\end{array}$

C $2.744531 .29245-1.40167$

$\begin{array}{llll}\text { C } & 4.10147 & 1.66949 & -0.76142\end{array}$

B $\quad 0.79182 \quad 1.74773 \quad 0.34327$

$\begin{array}{llll}\text { C } & 0.56581 & 2.99009 & 1.30669\end{array}$

C $1.31547 \quad 4.25367 \quad 0.81140$

$\begin{array}{llll}\text { C } & -0.93130 & 3.33290 & 1.47469\end{array}$

C $\quad 1.153172 .575962 .68675$

C $-2.64809-1.40804 \quad 1.78615$

C $-3.86272-2.334351 .99342$

$\begin{array}{llll}\text { C } & -3.68269 & 0.89617 & 0.03870\end{array}$

$\begin{array}{llll}C & -5.08809 & 0.63007 & 0.61450\end{array}$

C $-2.52397-0.38043 \quad 2.92931$

C $2.91307 \quad 0.07053-2.32636$

C $2.216802 .47960-2.24302$

C $-3.769461 .51018-1.37143$

C $-2.01945-3.05992-1.12413$

H $-1.74628-2.048221 .80937$

H $-3.17976 \quad 1.63317 \quad 0.69174$

H $-2.56691-1.26135-2.15415$

H $\quad 0.14153-1.135692 .29646$

H $-4.345462 .45236-1.32294$

H -2.77500 $1.71803-1.79473$

H $-4.292530 .83988-2.07535$

H $-5.67554 \quad 1.565390 .56386$

H $-5.63995-0.131180 .04035$

$\begin{array}{llll}\mathrm{H} & -5.06930 & 0.31497 & 1.66924\end{array}$

H - $-2.14205-3.70151-2.01507$

H $-0.95336-2.78221-1.05483$

H - $-2.27381-3.67089-0.23977$

H $-4.49102-2.86867-2.29577$

H $-4.83847-2.66368-0.56458$

H -5.00983 - $1.28651-1.68429$

H $-2.41327-0.902743 .89725$

H $-1.65271 \quad 0.281252 .79444$

H $-3.422340 .25527 \quad 3.00438$

H $-3.81990-2.76407 \quad 3.01171$ 
$\begin{array}{crrr}\mathrm{H} & -4.82514 & -1.80733 & 1.90147 \\ \mathrm{H} & -3.86762 & -3.17720 & 1.28557 \\ \mathrm{H} & 4.82497 & 1.94243 & -1.54961 \\ \mathrm{H} & 3.99332 & 2.53491 & -0.08514 \\ \mathrm{H} & 4.52033 & 0.82838 & -0.18689 \\ \mathrm{H} & 2.91747 & 2.68545 & -3.07219 \\ \mathrm{H} & 1.22954 & 2.23505 & -2.66639 \\ \mathrm{H} & 2.13892 & 3.39602 & -1.63578 \\ \mathrm{H} & 3.59053 & 0.33396 & -3.15700 \\ \mathrm{H} & 3.35174 & -0.78618 & -1.79381 \\ \mathrm{H} & 1.93822 & -0.22195 & -2.74830 \\ \mathrm{H} & 1.20278 & 5.06864 & 1.55218 \\ \mathrm{H} & 2.39584 & 4.07027 & 0.68156 \\ \mathrm{H} & 0.91208 & 4.62004 & -0.14805 \\ \mathrm{H} & 0.99208 & 3.38857 & 3.42081 \\ \mathrm{H} & 0.67238 & 1.66350 & 3.07916 \\ \mathrm{H} & 2.23808 & 2.38411 & 2.62387 \\ \mathrm{H} & -1.05662 & 4.18701 & 2.16767 \\ \mathrm{H} & -1.39381 & 3.60575 & 0.51068 \\ \mathrm{H} & -1.49040 & 2.47686 & 1.88806 \\ \mathrm{H} & 2.09070 & -2.96923 & -0.64769 \\ \mathrm{H} & 4.13600 & -4.40860 & -0.69212 \\ \mathrm{H} & 6.13822 & -3.78017 & 0.67181 \\ \mathrm{H} & 6.09090 & -1.71101 & 2.07821 \\ \mathrm{H} & 4.05699 & -0.25379 & 2.08989\end{array}$

TS(B2-C2)

$\mathrm{SCF}$ Energy $=-1191.68602476$

Enthalpy $0 \mathrm{~K}=-1191.051893$

Enthalpy $298 \mathrm{~K}=-1191.010727$

Free Energy $298 \mathrm{~K}=-1191.122402$

$\mathrm{SCF}(\mathrm{C} 6 \mathrm{H} 6)=-1191.69059528$

$\mathrm{SCF}(\mathrm{BP} 86-\mathrm{D} 3)=-0.13443107$

Lowest Frequencies $=-182.383617 .3654 \mathrm{~cm}-1$

C - $1.05130 \quad 0.89417-0.90102$

Rh $0.08025-0.78995-0.17151$

N $-1.86438-1.66450-0.30938$

C -2.29318 $-2.87484-0.99707$

$\begin{array}{llll}\text { C }-2.39279 & -4.05599 & 0.00149\end{array}$

C $-0.389040 .24335-1.81653$

B $-2.07995-0.53506 \quad 0.34902$

C $-2.952450 .03039 \quad 1.57830$

$\begin{array}{llll}C & -4.08686 & 0.99506 & 1.15365\end{array}$

$\begin{array}{lllll}\text { P } & 2.30594 & 0.15596 & 0.00132\end{array}$

C $2.523901 .11054 \quad 1.64261$

C 3.771062 .003991 .79380

Cl $0.78757-2.687451 .08252$

C -3.60974-1.23457 2.20502

C -2.084120 .688392 .67750$

C $3.56668-1.27189-0.03726$

C $5.00582-1.014460 .44376$

C $2.779211 .34320-1.42991$

C $4.28237 \quad 1.55743-1.69848$

C $3.51403-1.95534-1.41876$

C $2.051812 .69747-1.33276$

$\begin{array}{llll}\text { C } & 2.37446 & 0.15236 & 2.84160\end{array}$

C $-3.68362-2.59760-1.62124$

C $-1.29189-3.23951-2.11818$

H $2.355340 .80417-2.29990$

H $1.63251 \quad 1.767091 .62634$

H $3.08395-1.967110 .67396$

C - $1.558362 .25297-0.68563$ 
$\begin{array}{llll}\mathrm{H} & -0.13312 & 0.13793 & -2.86922\end{array}$

$\begin{array}{llll}\mathrm{H} & 2.30080 & 0.73973 & 3.77460\end{array}$

H $1.48174-0.486782 .75675$

H $3.24918-0.513012 .93844$

H 3.723062 .524772 .76800

H $4.70598 \quad 1.42195 \quad 1.78221$

H $3.841662 .77717 \quad 1.01344$

H $4.08982-2.89764-1.38734$

H $2.47726-2.21161-1.69604$

H $3.94537-1.33137-2.22092$

H $5.56430-1.968340 .40919$

H $5.55618-0.29111-0.17747$

H $5.03550-0.662141 .48683$

H $2.23375 \quad 3.27669-2.25632$

H $0.964732 .58699-1.21868$

H $2.424503 .30686-0.49239$

H $4.397972 .24987-2.55294$

H $4.803472 .00928-0.83957$

H $4.804700 .62613-1.96299$

H $-4.05397-3.49627-2.14507$

H $-4.41663-2.32951-0.84180$

H -3.62789-1.76735 -2.34539

H -2.70836 -4.97296 - 0.52806

$\begin{array}{llll}\text { H } & -1.41683 & -4.23241 & 0.47868\end{array}$

H $-3.13237-3.839540 .78957$

H - $1.63029-4.14713-2.64770$

H - $1.20887-2.42020-2.85237$

H $-0.29494-3.43215-1.68986$

H $-4.21154-0.939513 .08651$

H $-4.28498-1.740301 .49388$

H -2.85145 -1.96483 2.53264

$\begin{array}{llll}\mathrm{H} & -4.72619 & 1.22141 & 2.02871\end{array}$

H $-3.70424 \quad 1.94943 \quad 0.76313$

$\begin{array}{llll}\mathrm{H} & -4.73338 & 0.54495 & 0.37973\end{array}$

H -2.708340 .904693 .56615$

H -1.263740 .022282 .99529$

H -1.646841 .640692 .34314$

C -1.090843 .094160 .35116$

$\begin{array}{llll}C & -1.56109 & 4.41026 & 0.47301\end{array}$

C $-2.50865 \quad 4.91352-0.43238$

C -2.97741 4.08836-1.47004

C $-2.512832 .77342-1.59697$

H -0.341362 .710961 .04949$

$\begin{array}{llll}\mathrm{H} & -1.18231 & 5.04471 & 1.28147\end{array}$

H $-2.878975 .93863-0.33164$

H $-3.715754 .46953-2.18363$

H $-2.889052 .12799-2.39695$

\section{TS(B2-C4)}

SCF Energy $=-1191.66713904$

Enthalpy $0 \mathrm{~K}=-1191.032391$

Enthalpy $298 \mathrm{~K}=-1190.991528$

Free Energy 298K = -1191.102293

$\mathrm{SCF}(\mathrm{C} 6 \mathrm{H} 6)=-1191.67184267$

$\mathrm{SCF}(\mathrm{BP} 86-\mathrm{D} 3)=-0.13469478$

Lowest Frequencies $=-324.417125 .1744 \mathrm{~cm}-1$

C -0.01762 $1.71681-0.62619$

Rh $-0.56377-0.29942-0.50062$

$\begin{array}{llll}\mathrm{N} & -2.53987 & 0.51241 & -0.37790\end{array}$

C $-3.796190 .29250-1.15379$

C $-4.52711-0.96018-0.61754$

C $-1.14086 \quad 1.58721-1.28513$ 
$\begin{array}{llll}\text { B } & -2.01872 & 0.14653 & 0.85679\end{array}$

$\begin{array}{llll}\text { C } & -2.26258 & 0.29159 & 2.42041\end{array}$

$\begin{array}{llll}\text { C } & -1.95103 & 1.77168 & 2.78050\end{array}$

P $1.57290-1.260000 .06629$

$\begin{array}{llll}\text { C } & 2.86143 & 0.04678 & 0.56698\end{array}$

C $4.35677-0.312290 .47946$

Cl -1.28007 -2.47477-1.33516

C $-3.73818-0.007252 .79383$

C $-1.34451-0.632393 .25017$

C $1.50510-2.532061 .50208$

C $2.85302-2.906592 .15360$

C $2.19825-2.15809-1.49215$

C $3.37960-3.13538-1.35360$

C $0.71056-3.79873 \quad 1.13163$

C $2.42905-1.12925-2.61707$

C $\quad 2.48298 \quad 0.65655 \quad 1.92983$

$\begin{array}{llll}C & -4.67372 & 1.55135 & -0.95561\end{array}$

C $-3.507060 .07874-2.65700$

H $1.29354-2.73843-1.75572$

H $2.67093 \quad 0.82704-0.19009$

H $0.91258-1.976212 .25111$

C $1.008112 .74833-0.45773$

H - $1.751141 .96369-2.09949$

H $3.06217 \quad 1.580902 .09991$

H $1.412990 .92119 \quad 1.95843$

H $2.69395-0.02902 \quad 2.76841$

$\begin{array}{lllll}\mathrm{H} & 4.95334 & 0.57467 & 0.76199\end{array}$

H $4.64581-1.13218 \quad 1.15414$

H $4.65580-0.59192-0.54312$

H $\quad 0.54715-4.399332 .04522$

$\begin{array}{llll}\mathrm{H} & -0.26311 & -3.56357 & 0.67665\end{array}$

H $1.26402-4.43307 \quad 0.41783$

H $2.66157-3.639402 .95900$

H $3.55005-3.37807 \quad 1.44296$

H $3.36319-2.046732 .61357$

H 2.61583-1.65511-3.57046

H $1.54621-0.48235-2.76026$

H $3.30319-0.48361-2.41910$

H $3.56749-3.61164-2.33380$

H $4.31512-2.64194-1.04500$

H $3.16688-3.94395-0.63714$

H $-5.623941 .44419-1.50743$

$\begin{array}{llll}\mathrm{H} & -4.91180 & 1.70308 & 0.11076\end{array}$

H $-4.159282 .45542-1.32239$

H $-5.46495-1.11509-1.18063$

H $-3.88888-1.84989-0.73743$

H $-4.78994-0.842880 .44546$

H -4.44298 - $0.21536-3.16161$

H $-3.148280 .99373-3.15722$

H -2.76712 - $0.72580-2.79322$

H $-3.89013 \quad 0.167413 .87629$

$\begin{array}{llll}\mathrm{H} & -4.44432 & 0.64485 & 2.25158\end{array}$

H -4.00903 - 1.056552 .58636

H $-2.11255 \quad 1.935993 .86314$

H -0.901782 .028802 .55582$

H $-2.600232 .47302 \quad 2.22875$

H $-1.53490-0.493894 .33175$

H - $1.51391-1.695533 .00810$

H $-0.28180-0.404423 .06454$

C 0.931163 .686130 .60093

C $1.88574 \quad 4.70554 \quad 0.72737$

C $2.942034 .80966-0.19299$ 
C $3.030103 .88876-1.25005$

C $2.074822 .87062-1.38420$

H $0.10501 \quad 3.614351 .31482$

$\mathrm{H} \quad 1.80184 \quad 5.42430 \quad 1.54976$

H $3.686685 .60578-0.09109$

H $3.841483 .96784-1.98168$

H $2.134272 .16486-2.21946$

Intermediate $\mathrm{C} 1$

SCF Energy $=-1191.72632821$

Enthalpy 0K $=-1191.090094$

Enthalpy $298 \mathrm{~K}=-1191.049272$

Free Energy 298K = -1191.160000

$\mathrm{SCF}(\mathrm{C} 6 \mathrm{H} 6)=-1191.73495254$

$\mathrm{SCF}(\mathrm{BP} 86-\mathrm{D} 3)=-013124061$

Lowest Frequencies $=22.947228 .0537 \mathrm{~cm}-1$

C $-0.93236 \quad 4.806321 .89806$

$\begin{array}{llll}C & -0.96193 & 3.40892 & 1.92053\end{array}$

$\begin{array}{llll}C & -0.54750 & 2.66449 & 0.77961\end{array}$

C $-0.141973 .36365-0.38999$

C $-0.144844 .76192-0.40934$

$\begin{array}{llll}\text { C } & -0.52651 & 5.48719 & 0.73468\end{array}$

$\begin{array}{llll}\text { C } & -0.56889 & 1.22754 & 0.81402\end{array}$

$\begin{array}{llll}\text { C } & -1.08700 & 0.18040 & 1.55705\end{array}$

Rh $-0.19478-0.17911-0.42819$

$\begin{array}{llll}\text { P } & 2.16662 & -0.58472 & 0.03349\end{array}$

C $3.355250 .38000-1.09688$

C $4.79909-0.12916-1.25615$

Cl $0.359510 .19292-2.72173$

N -2.18931 - $0.52674-0.55661$

C $-3.16111-0.40878-1.65122$

C $-4.60160-0.57847-1.11230$

B $-2.10937-0.845180 .82368$

C $-2.76461-2.05579 \quad 1.70310$

C $-3.00504-3.312160 .83032$

C $-1.84089-2.484482 .87030$

C $-4.10617-1.584192 .32602$

C $2.62772-0.22231 \quad 1.85162$

C $4.11948-0.053532 .19905$

C $2.45030-2.45669-0.24679$

C $3.75598-3.076740 .28923$

C $1.96061-1.240522 .79715$

C $-3.05477 \quad 1.00910-2.26996$

C $-2.88175-1.47860-2.73522$

C $2.22827-2.82310-1.72854$

C $3.310461 .88040-0.74701$

$\begin{array}{llll}\text { H } & 2.12699 & 0.75335 & 2.00204\end{array}$

H $1.60858-2.883140 .33326$

H $2.83017 \quad 0.26196-2.06376$

H $-0.77452-0.008122 .59352$

H $2.20755-3.92242-1.83699$

H $1.28577-2.41483-2.12630$

H $3.04398-2.44262-2.36688$

H $3.73109-4.168860 .11863$

H $4.64674-2.68638-0.22659$

H $3.89325-2.91937 \quad 1.37015$

H $3.807612 .45887-1.54621$

H $2.276302 .24926-0.66400$

H 3.832512 .111650 .19758

H $5.330720 .52592-1.97140$

H $5.36967-0.11303-0.31342$

H $4.83888-1.15050-1.66571$ 
H $2.00261-0.868983 .83695$

H $\quad 0.90522-1.417652 .54158$

H $2.48005-2.213252 .77870$

$\mathrm{H} \quad 4.21310 \quad 0.21685 \quad 3.26733$

H $4.69215-0.982942 .04814$

$\begin{array}{llll}\text { H } & 4.60464 & 0.74402 & 1.61698\end{array}$

H -5.32322 - $0.41486-1.93137$

H $-4.77788-1.58820-0.70930$

H $-4.810600 .15945-0.31909$

H -3.60384 - $1.38229-3.56551$

H $-1.86463-1.35471-3.13874$

H $-2.97497-2.49254-2.31033$

H $-3.78031 \quad 1.11846-3.09583$

$\mathrm{H}-3.27803 \quad 1.77558-1.50787$

H $-2.04157 \quad 1.18174-2.66218$

H $-3.45228-4.12671 \quad 1.43332$

H $-3.68383-3.11447-0.01468$

H $-2.05860-3.69088 \quad 0.40465$

$\mathrm{H}-4.54182-2.382932 .95840$

H -3.96155 -0.69602 2.96757

H $-4.85415-1.32274 \quad 1.56048$

H $-2.29443-3.32712 \quad 3.42713$

H $-0.85310-2.82523 \quad 2.51085$

H - $1.67714-1.671783 .60054$

H $\quad 0.133782 .78007-1.27530$

H $0.15143 \quad 5.29191-1.32037$

H $-0.51833 \quad 6.58194 \quad 0.71728$

$\begin{array}{llll}\mathrm{H} & -1.24251 & 5.37083 & 2.78358\end{array}$

H - 1.300612 .872282 .81262

Intermediate $\mathrm{C} 2$

SCF Energy $=-1191.68965625$

Enthalpy 0K $=-1191.054020$

Enthalpy $298 \mathrm{~K}=-1191.013186$

Free Energy 298K = -1191.124019

SCF $($ C6H6) $=-1191.6945878$

$\mathrm{SCF}(\mathrm{BP} 86-\mathrm{D} 3)=-0.13281001$

Lowest Frequencies $=16.908220 .2382 \mathrm{~cm}-1$

C $-0.98322 \quad 2.40354-0.28111$

C $-1.197643 .37915-1.28123$

C $-0.95508 \quad 4.73665-1.01611$

$\begin{array}{llll}C & -0.47763 & 5.13767 & 0.24174\end{array}$

$\begin{array}{llll}C & -0.25127 & 4.17211 & 1.23824\end{array}$

$\begin{array}{llll}C & -0.50181 & 2.81667 & 0.98156\end{array}$

C $-1.263270 .95701-0.57631$

Rh $0.00496-0.80485-0.09608$

P $2.37226-0.21705-0.03346$

C $3.38158-1.829490 .03898$

C $3.15296-2.63041-1.25968$

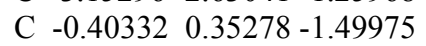

B $-2.518520 .01654-0.06197$

$\begin{array}{llll}\text { C } & -3.92820 & 0.68210 & 0.42147\end{array}$

C $-3.70913 \quad 1.95975 \quad 1.27893$

N $-2.04608-1.29650-0.25313$

C $-2.63788-2.63924-0.43926$

C $-1.64728-3.54861-1.21118$

Cl $0.46840-2.65659 \quad 1.33202$

$\begin{array}{llll}\text { C } & 2.82427 & 0.86782 & 1.47082\end{array}$

C $4.22401 \quad 1.51163 \quad 1.51188$

C $2.940780 .73376-1.59639$

C $4.435590 .66668-1.96610$

C 2.519390 .102312 .77450 
C $2.460882 .19769-1.56610$

C $4.86738-1.769630 .44003$

C $-3.88977-2.50521-1.34635$

C $-3.00783-3.319660 .90228$

C $-4.80259-0.25283 \quad 1.29442$

C $-4.73042 \quad 1.10628-0.84120$

H $3.24068-0.71622 \quad 2.94161$

H $1.51182-0.343502 .76874$

H $2.59570 \quad 0.790693 .63559$

H $2.07840 \quad 1.68049 \quad 1.37292$

H $5.02877 \quad 0.76255 \quad 1.56680$

H 4.422382 .160420 .64467

H 4.303032 .143072 .41616

H $3.068072 .80650-0.87560$

$\begin{array}{llll}\mathrm{H} & 2.56417 & 2.64622 & -2.57069\end{array}$

H $2.376390 .20161-2.38658$

H $4.602841 .23959-2.89703$

H 5.07841 1.11119-1.18952

H $4.78202-0.36157-2.14892$

H $5.00804-1.369771 .45668$

H $5.48995-1.17883-0.24987$

H $5.27084-2.79940 \quad 0.44347$

H $3.64297-2.17121-2.13567$

H $2.07729-2.73752-1.47870$

H $3.57098-3.64634-1.14326$

H $2.83262-2.36370 \quad 0.83535$

H - $1.34235-3.06283-2.15485$

H $-0.74718-3.75855-0.61628$

H $-2.13550-4.50855-1.45588$

H -2.11292 -3.407751 .53658$

H $-3.40911-4.332530 .71730$

H $-3.76974-2.743551 .44838$

H $-3.61290-2.05685-2.31620$

H -4.67644 - $1.88568-0.89301$

H $-4.31742-3.50519-1.53771$

H $-4.27086-0.556772 .21305$

H -5.12120 - 1.169790 .77610

H -5.723120 .278961 .60526$

$\begin{array}{llll}\mathrm{H} & -3.08421 & 1.76131 & 2.16814\end{array}$

H $-3.241512 .77890 \quad 0.71309$

H $-4.686962 .33067 \quad 1.64346$

H $-5.671801 .61314-0.55059$

H $-4.99880 \quad 0.24443-1.47562$

H -4.15592 $1.81514-1.46504$

H -0.345912 .062421 .75977$

$\begin{array}{lllll}\mathrm{H} & 0.11472 & 4.47722 & 2.22447\end{array}$

H $-0.28573 \quad 6.19583 \quad 0.44639$

H -1.13759 5.48126-1.79822

H - $1.574723 .07008-2.26229$

H $-0.025140 .61672-2.49724$

H $1.409642 .30526-1.25712$

Intermediate C3

SCF Energy $=-1191.71846611$

Enthalpy 0K $=-1191.081479$

Enthalpy 298K $=-1191.040776$

Free Energy 298K = -1191.151037

$\mathrm{SCF}(\mathrm{C} 6 \mathrm{H} 6)=-1191.72428396$

$\mathrm{SCF}(\mathrm{BP} 86-\mathrm{D} 3)=-0.13361376$

Lowest Frequencies $=23.298725 .9307 \mathrm{~cm}-1$

C $\quad-5.45185 \quad 1.72748 \quad 1.11856$

$\begin{array}{llll}\text { C } & -4.29720 & 0.95669 & 0.92644\end{array}$ 
C $-3.26776 \quad 1.39400 \quad 0.05765$

C $-3.437852 .63473-0.60485$

C $-4.589623 .40848-0.40448$

C $-5.60505 \quad 2.95658 \quad 0.45420$

C $-1.985380 .67176-0.09990$

C $-0.73135 \quad 1.199330 .00717$

Rh $0.45641-0.29613-0.56880$

$\begin{array}{lllll}\text { P } & 2.48234 & 0.59756 & 0.06841\end{array}$

C $3.373431 .02570-1.56203$

C $4.87418 \quad 1.36742-1.51331$

Cl $1.42261-1.88487-2.13053$

$\mathrm{N}-1.86448-0.79730-0.24646$

C $-2.68141-1.57412-1.26596$

C $-4.11812-1.84126-0.75899$

B $-0.76440-1.259510 .64039$

C $-0.76673-2.091872 .00283$

C $-1.47665-3.46563 \quad 1.89445$

C $0.65651-2.322402 .55482$

C $-1.55819-1.214623 .01987$

C $2.27441 \quad 2.20772 \quad 1.07033$

C 3.467873 .179811 .15111

C $3.59536-0.584391 .07029$

C 4.782020 .054661 .81896

C $\quad 1.68864 \quad 1.897102 .46143$

C $-2.72132-0.76194-2.57686$

C $-1.98776-2.92346-1.53663$

C $4.04739-1.787210 .22126$

C $2.559762 .09177-2.32355$

$\mathrm{H} \quad 1.48313 \quad 2.71070 \quad 0.48415$

H $2.87796-0.95762 \quad 1.82147$

H $3.247920 .06737-2.10189$

H $-0.55806 \quad 2.24467 \quad 0.29394$

H $4.49823-2.547640 .88426$

H $3.21042-2.24430-0.32982$

H $4.81557-1.49933-0.51685$

H $5.32789-0.740312 .35944$

H $\begin{array}{llll}5.50130 & 0.53849 & 1.13893\end{array}$

H $4.46478 \quad 0.796862 .56806$

H $2.961322 .20627-3.34616$

H $1.49710 \quad 1.80441-2.41190$

H $2.609323 .08156-1.83595$

H $5.239761 .51350-2.54658$

H $5.088752 .29417-0.95788$

H $5.473640 .55656-1.07084$

$\mathrm{H} \quad 1.385822 .835962 .95918$

H $\quad 0.80073 \quad 1.24847 \quad 2.38500$

H $2.42576 \quad 1.402253 .11712$

H $3.168374 .06691 \quad 1.73974$

H $4.346912 .74009 \quad 1.64657$

H 3.779773 .540200 .15863

H $-4.63075-2.51044-1.47160$

H $-4.10768-2.343830 .22345$

H $-4.71002-0.91836-0.67461$

H $-2.54780-3.45930-2.32199$

H $-0.95133-2.78163-1.88577$

H $-1.98269-3.56706-0.64355$

H $-3.28174-1.32652-3.34110$

H $-3.218540 .21205-2.44014$

H $-1.69850-0.59124-2.95363$

H - $1.51126-3.939452 .89411$

H $-2.51683-3.375141 .53797$

H $-0.93706-4.15314 \quad 1.22141$ 
H $-1.54940-1.703104 .01302$

H -1.11636 -0.20989 3.13569

H -2.61068-1.08777 2.71604

H $0.61545-2.904683 .49532$

H $\quad 1.28637-2.87243 \quad 1.83584$

H $1.14902-1.361962 .78055$

H -2.65309 2.97561 -1.28855

H $-4.700014 .36221-0.93176$

H $-6.50887 \quad 3.555860 .60489$

H $-6.231821 .37211 \quad 1.80071$

H -4.177130 .014831 .47009$

Intermediate $\mathrm{C} 4$

SCF Energy $=-1191.72180892$

Enthalpy 0K $=-1191.084505$

Enthalpy $298 \mathrm{~K}=-1191.043644$

Free Energy 298K = -1191.154378

SCF $($ C6H6) $=-1191.727082$

$\mathrm{SCF}(\mathrm{BP} 86-\mathrm{D} 3)=-0.13600347$

Lowest Frequencies $=27.079930 .8290 \mathrm{~cm}-1$

C $-0.52002 \quad 1.63689-0.18112$

$\mathrm{Rh}-0.38790-0.39250-0.54288$

N $-2.52526 \quad 0.43980-0.25487$

C $-3.749540 .21130-1.10985$

C $-4.12726-1.28056-1.09309$

C $-1.869721 .73335-0.31938$

B $-1.84575-0.40403 \quad 0.76499$

$\begin{array}{llll}\text { C }-2.26811 & -0.81301 & 2.24816\end{array}$

C $-2.34880 \quad 0.53749 \quad 3.02410$

$\begin{array}{llll}\text { P } & 1.78502 & -0.96009 & 0.00870\end{array}$

C 2.739430 .460290 .84729

$\begin{array}{llll}\text { C } & 4.28001 & 0.43577 & 0.81285\end{array}$

Cl $-0.71052-2.35349-1.93339$

C $-3.65149-1.505702 .34201$

C $-1.22669-1.718652 .93918$

C $1.90899-2.486991 .15791$

C $3.26224-2.706911 .86456$

C $2.65559-1.43030-1.62344$

C $4.03340-2.11366-1.55038$

C $1.43170-3.77504 \quad 0.46080$

C $2.66419-0.22535-2.58301$

C $2.18563 \quad 0.70266 \quad 2.26475$

C $-4.911821 .07126-0.56107$

C $-3.409410 .61952-2.55975$

H $1.92264-2.15840-2.02043$

H $2.41475 \quad 1.31860 \quad 0.23326$

H $1.16458-2.23236 \quad 1.93081$

C $0.346752 .82093-0.07723$

H -2.48638 $2.62935-0.47505$

$\begin{array}{lllll}\mathrm{H} & 2.55915 & 1.66778 & 2.64998\end{array}$

$\begin{array}{lllll}\mathrm{H} & 1.08413 & 0.74833 & 2.25558\end{array}$

H $2.49572-0.081352 .97689$

H $4.66146 \quad 1.34272 \quad 1.31719$

H $4.71490 \quad-0.43427 \quad 1.32863$

H $4.668120 .45109-0.21774$

H $1.33121-4.57703 \quad 1.21483$

H $\quad 0.46449-3.64136-0.04815$

H $2.15767-4.12577-0.29291$

H $3.17703-3.593822 .51912$

H $4.08293-2.903261 .15686$

H $3.55566-1.859192 .50217$

H $3.05198-0.53722-3.56952$ 
H $\quad \begin{array}{llll}1.64547 & 0.16980 & -2.73944\end{array}$

H $3.306380 .59631-2.21908$

H $4.35430-2.37741-2.57518$

H $4.81421-1.46730-1.11981$

H $4.00559-3.04913-0.97084$

H $-5.81450 \quad 0.91153-1.17512$

$\begin{array}{llll}\mathrm{H} & -5.15391 & 0.79658 & 0.47930\end{array}$

H $-4.679282 .14935-0.58888$

H $-4.96565-1.44548-1.79096$

H -3.27605-1.90082-1.42077

H $-4.45797-1.61192-0.09803$

H $-4.28598 \quad 0.45170-3.20823$

$\mathrm{H}-3.127951 .68306-2.63205$

H $-2.572340 .00796-2.93668$

H -3.88998 -1.70365 3.40452

H $-4.46232-0.877221 .93697$

H $-3.66423-2.47405 \quad 1.81375$

$\mathrm{H}-2.62772 \quad 0.33874 \quad 4.07651$

H $-1.38180 \quad 1.068793 .02707$

$\mathrm{H}-3.106841 .213762 .59358$

H - $1.54595-1.952543 .97292$

H - $1.09886-2.671602 .39859$

H - $0.24554-1.219322 .99899$

$\begin{array}{llll}\text { C } & 0.16619 & 3.75978 & 0.97009\end{array}$

C $0.95136 \quad 4.92028 \quad 1.04539$

C $1.93914 \quad 5.17259 \quad 0.07947$

C $2.13035 \quad 4.25493-0.96814$

C $1.348183 .09423-1.04411$

H -0.600473 .562851 .72699$

H $0.789925 .63085 \quad 1.86363$

H $2.55213 \quad 6.07787 \quad 0.13956$

H $2.887674 .44920-1.73561$

H $1.484602 .39244-1.87322$

TS(C1-3)

SCF Energy $=-1191.71565602$

Enthalpy 0K $=-1191.080159$

Enthalpy $298 \mathrm{~K}=-1191.039888$

Free Energy 298K = -1191.149786

$\mathrm{SCF}(\mathrm{C} 6 \mathrm{H} 6)=-1191.72012108$

$\mathrm{SCF}(\mathrm{BP} 86-\mathrm{D} 3)=-0.12954624$

Lowest Frequencies $=-132.922817 .5476 \mathrm{~cm}-1$

C $2.075874 .48687-1.25066$

C $1.829243 .11702-1.39278$

C $1.087262 .41811-0.40311$

$\begin{array}{lllll}\text { C } & 0.62521 & 3.11842 & 0.74063\end{array}$

$\begin{array}{llll}\text { C } & 0.88899 & 4.48385 & 0.88370\end{array}$

C $1.608605 .17003-0.11305$

C $0.823921 .00743-0.56698$

C $1.13069-0.01896-1.47680$

B $2.19583-0.93580-0.71916$

C $3.22624-1.87885-1.53913$

C $4.39210-0.99031-2.05634$

Rh $-0.16600-0.396310 .22651$

Cl -0.97304 -1.58963 2.14024

N $1.93996-0.577190 .64893$

C $2.63470-0.75007 \quad 1.93664$

C $2.53261-2.201122 .47208$

P $-2.47132-0.19831-0.23590$

C $-3.30402-1.91109-0.41607$

C -2.38649-2.93345-1.11312

C $4.12016-0.349031 .75194$ 
C $\quad 2.01379 \quad 0.21457 \quad 2.97640$

C $3.81526-3.08149-0.76593$

C $2.48143-2.45584-2.77513$

$\begin{array}{llll}\text { C } & -3.56009 & 0.70715 & 1.07212\end{array}$

$\begin{array}{llll}C & -2.73680 & 1.77529 & 1.81544\end{array}$

C $-2.993660 .74143-1.81688$

C $-2.632412 .23563-1.73971$

C $-4.26864-0.21002 \quad 2.08705$

$\begin{array}{llll}C & -2.40245 & 0.10565 & -3.08699\end{array}$

C $-4.70890-1.87583-1.04475$

H $-4.096850 .65130-1.85267$

$\begin{array}{llll}\mathrm{H} & -3.38642 & -2.22301 & 0.64001\end{array}$

$\begin{array}{llll}\mathrm{H} & -4.33278 & 1.21433 & 0.46072\end{array}$

H $0.839590 .02471-2.53395$

H $3.04175-2.278313 .45013$

H $1.47665-2.482062 .60147$

H $3.00691-2.916191 .78334$

H $2.54456 \quad 0.12593 \quad 3.94060$

$\begin{array}{llll}\mathrm{H} & 2.10087 & 1.25881 & 2.62994\end{array}$

H $0.95084-0.021793 .13861$

H $4.65316-0.414792 .71681$

H $4.63664-1.008151 .03662$

H $4.19706 \quad 0.68857 \quad 1.38395$

H $4.46358-3.68316-1.43294$

H $4.43342-2.773890 .09307$

H $3.02174-3.74877-0.38655$

H $3.15951-3.10029-3.36742$

H $1.61349-3.07118-2.47777$

H $2.11506-1.66178-3.44870$

H $5.06790-1.57653-2.70986$

H $4.01972-0.13548-2.65020$

H $4.99919-0.58256-1.22945$

H $-3.395612 .35622 \quad 2.48713$

H -1.95698 1.292522 .42806

H -2.244612 .482591 .12917$

H -4.860680 .415612 .78032$

H -4.96538 - 0.919531 .61352

H -3.53678 -0.780092 .68089$

H - $2.84707-3.93730-1.06261$

H -2.22403 -2.69698 -2.17806

H -1.40249 -2.98232 -0.61981

H $-5.18550-2.86825-0.94730$

H $-5.37735-1.14129-0.56425$

H -4.67084 - $1.64127-2.12263$

H -2.69449 $0.69514-3.97546$

H - $1.30093 \quad 0.08384-3.04271$

H $-2.75023-0.92722-3.24436$

H $-2.936702 .74247-2.67382$

H $-3.137292 .75108-0.90676$

H $-1.545152 .37981-1.61960$

$\begin{array}{lllll}\mathrm{H} & 0.07658 & 2.55911 & 1.50346\end{array}$

$\begin{array}{lllll}\mathrm{H} & 0.53813 & 5.01887 & 1.77191\end{array}$

$\begin{array}{lllll}\mathrm{H} & 1.81303 & 6.23978 & 0.00140\end{array}$

H $2.642475 .02328-2.01850$

H $2.203182 .56753-2.26244$

Intermediate 3

SCF Energy $=-1191.75176586$

Enthalpy 0K $=-1191.113349$

Enthalpy $298 \mathrm{~K}=-1191.073115$

Free Energy 298K = -1191.182009

SCF $($ C6H6) $=-1191.75631946$ 
$\mathrm{SCF}(\mathrm{BP} 86-\mathrm{D} 3)=-0.13550444$

Lowest Frequencies $=27.570634 .9651 \mathrm{~cm}-1$ Rh $0.19357-0.35015-0.34921$

H $-0.43302 \quad 0.083362 .48353$

Cl $0.88470-1.08280-2.50693$

$\begin{array}{lllll}\text { P } & 2.35809 & 0.30052 & 0.16254\end{array}$

N - $1.95153-0.61973-0.27120$

B $-1.38204-1.553470 .83158$

$\begin{array}{llll}\text { C } & -1.46835 & 0.53617 & 0.51770\end{array}$

C $-0.86972-0.27005 \quad 1.55106$

$\begin{array}{llll}C & -1.94434 & 1.94639 & 0.52044\end{array}$

C $-2.900362 .33600 \quad 1.48645$

H $-3.29198 \quad 1.587702 .18382$

C $-1.428812 .91954-0.36117$

H $-0.669992 .61947-1.09008$

C $-1.87048 \quad 4.24926-0.28915$

H $-1.459734 .99524-0.97766$

C $-2.83149 \quad 4.62462 \quad 0.66425$

H $-3.17473 \quad 5.662990 .71941$

C $-3.344413 .66530 \quad 1.55275$

H $-4.08785 \quad 3.95258 \quad 2.30375$

C $-1.49373-3.10209 \quad 1.20568$

C $-0.91034-4.05628 \quad 0.13231$

H $0.14174-3.81288-0.09272$

H - $1.46262-4.00922-0.81872$

H $-0.94975-5.102740 .49292$

C $-0.69805-3.312412 .52217$

H $-0.75406-4.370522 .84219$

H $-1.09684-2.691223 .34352$

H $\quad 0.36758-3.057462 .39398$

C $-2.97449-3.481891 .48499$

H $-3.03762-4.526641 .84602$

H $-3.60509-3.408630 .58222$

$\mathrm{H}-3.42346-2.836902 .26203$

C $-2.95343-0.64266-1.38130$

C $-4.35660-0.42670-0.76619$

H $-4.58605-1.20608-0.02028$

H -5.12403-0.47186-1.55791

H $-4.429220 .55900-0.27714$

C $-2.87380-2.01480-2.07446$

H - $1.85830-2.19111-2.46533$

H $-3.58409-2.03517-2.91832$

H $-3.14597-2.83231-1.38895$

C $-2.649360 .45198-2.42509$

$\mathrm{H}-2.76254 \quad 1.46352-2.00750$

H $-3.360640 .35380-3.26295$

H $-1.626600 .33100-2.81817$

C $2.71822 \quad 1.31020 \quad 1.74586$

H 3.771661 .629411 .62772

C $1.829382 .56344 \quad 1.82726$

H $\quad 0.760242 .29605 \quad 1.85852$

H 1.976993 .239140 .96996

H 2.064443 .133532 .74482

C $2.62217 \quad 0.48488 \quad 3.04189$

H $2.81249 \quad 1.141883 .91040$

H $3.36230-0.33013 \quad 3.08036$

H 1.620370 .043093 .17429

C $3.61806-1.143320 .33681$

H $4.43064-0.742450 .97528$

C $2.95964-2.339751 .05074$

H $2.14047-2.74667 \quad 0.43492$

H $2.53799-2.077242 .03330$ 
H $\quad 3.70540-3.14113 \quad 1.20545$

C $4.22019-1.60693-1.00349$

H $4.85562-2.49390-0.82570$

H $4.85534-0.84090-1.47678$

H $3.42849-1.89181-1.71700$

C $3.127321 .35255-1.23837$

H $3.268910 .58306-2.01764$

C $2.153392 .38795-1.82884$

H $1.906653 .19348-1.11636$

H $1.22027 \quad 1.89903-2.15221$

H $2.612732 .86143-2.71617$

C $4.486791 .98202-0.88728$

H $4.959622 .38611-1.80096$

H $5.192061 .25899-0.44315$

H $4.376542 .82469-0.18216$

TS(3-D1)

SCF Energy $=-1269.07107969$

Enthalpy $0 \mathrm{~K}=-1268.406957$

Enthalpy $298 \mathrm{~K}=-1268.362478$

Free Energy 298K = -1268.484017

SCF $($ C6H6) $=-1269.06007798$

$\mathrm{SCF}(\mathrm{BP} 86-\mathrm{D} 3)=-0.14790643$

Lowest Frequencies $=-8.369112 .7114 \mathrm{~cm}-1$

C $0.92232-0.24086 \quad 1.59875$

$\begin{array}{llll}\text { C } & 1.50531 & -0.90445 & 0.45782\end{array}$

$\begin{array}{lllll}\mathrm{N} & 2.10886 & 0.33103 & -0.09826\end{array}$

C $3.14100 \quad 0.47734-1.17274$

$\begin{array}{llll}C & 4.52761 & 0.24770 & -0.52737\end{array}$

Rh $-0.05623 \quad 0.20913-0.27983$

Cl $-0.62082 \quad 1.25983-2.35138$

$\begin{array}{llll}\mathrm{P}-2.24662 & -0.45413 & 0.15925\end{array}$

C $-2.75250-1.97876-0.88259$

C $-2.75318-1.63824-2.38596$

C $-2.50187-0.94282 \quad 1.99244$

C $-1.84328-2.30707 \quad 2.28740$

C $-3.93203-0.882332 .56220$

$\begin{array}{llll}\text { C } & -3.43878 & 0.97145 & -0.26799\end{array}$

$\begin{array}{llll}\text { C } & -3.14831 & 2.16962 & 0.65720\end{array}$

B $\quad 1.59096 \quad 1.09102 \quad 1.14777$

C $1.841172 .46235 \quad 1.92513$

C $2.273213 .71496 \quad 1.13230$

$\begin{array}{llll}\text { C } & 0.52948 & 2.79861 & 2.68509\end{array}$

C 2.942952 .149972 .98377

$\begin{array}{llll}C & -4.94581 & 0.67990 & -0.38699\end{array}$

C $-4.04676-2.71821-0.48626$

C $2.90443-0.53689-2.31044$

C $3.039761 .89583-1.76209$

C $-0.619394 .75786-1.64318$

C $-0.619365 .93456-1.32459$

H - $1.90400-0.161972 .49851$

H $-3.055991 .23902-1.27154$

H -1.90019-2.65803 -0.68681

$\begin{array}{llll}\mathrm{H} & 0.42867 & -0.70513 & 2.45040\end{array}$

C $1.84884-2.330220 .20407$

H $-0.613673 .70965-1.91602$

H $-0.62087 \quad 6.97152-1.04686$

H 3.105833 .032663 .63212

H 2.660601 .302993 .63343

H 3.910701 .904992 .51007

H 0.693943 .648623 .37493

H -0.274753 .083951 .98650$ 
$\begin{array}{llll}\mathrm{H} & 0.17146 & 1.94360 & 3.28519\end{array}$

H $2.31538 \quad 4.58812 \quad 1.81202$

$\begin{array}{lllll}\text { H } & 3.28355 & 3.60359 & 0.70267\end{array}$

H $1.57395 \quad 3.967300 .31745$

H $5.321150 .35716-1.28631$

$\begin{array}{llll}\mathrm{H} & 4.71661 & 0.98202 & 0.27404\end{array}$

H $4.60175-0.76647-0.09927$

H $3.792682 .00786-2.56074$

H $2.039612 .06039-2.19406$

H $3.232302 .66917-1.00643$

H $3.61469-0.32331-3.12702$

H $3.06746-1.57546-1.98737$

H $1.88134-0.43446-2.70855$

H -1.72944 -2.44070 3.37856

H -2.46079 -3.14446 1.92186

H $-0.84632-2.40299 \quad 1.82807$

H -3.90926 -1.15783 3.63305

H $-4.36626 \quad 0.126952 .49392$

H -4.61693 -1.58333 2.05894

$\begin{array}{llll}\mathrm{H} & -3.68568 & 3.06265 & 0.29203\end{array}$

$\mathrm{H}-3.47397 \quad 1.98784 \quad 1.69655$

$\begin{array}{llll}\mathrm{H} & -2.07160 & 2.40827 & 0.67047\end{array}$

H -5.46772 $1.61307-0.66944$

H -5.16619-0.06126-1.17124

$\begin{array}{llll}\mathrm{H} & -5.39532 & 0.32700 & 0.55480\end{array}$

H - $2.84258-2.56904-2.97530$

H -3.61123 -0.99762 -2.65379

H $-1.83986-1.10647-2.69579$

H -4.16912 -3.60140 - 1.14033

H $-4.03477-3.082520 .55233$

H $-4.94283-2.09140-0.61594$

C $2.77813-2.98001 \quad 1.04693$

C $3.07765-4.338010 .85865$

C $2.44508-5.06476-0.16343$

C $1.51122-4.42862-0.99866$

C $1.21455-3.06968-0.81670$

H $3.26122-2.414341 .85082$

H $3.80191-4.830521 .51593$

H $2.67637-6.12547-0.30618$

H $1.01287-4.99200-1.79459$

H $0.49329-2.55575-1.46169$

TS(3-D2)

SCF Energy $=-1191.72845310$

Enthalpy $0 \mathrm{~K}=-1191.092652$

Enthalpy $298 \mathrm{~K}=-1191.052160$

Free Energy 298K = -1191.162830

SCF $($ C6H6) $=-1191.73332125$

SCF $(B P 86-D 3)=-0.13386634$

Lowest Frequencies $=-140.635024 .0807 \mathrm{~cm}-1$

$\begin{array}{lllll}\mathrm{C} & 0.84787 & 0.33000 & 1.56857\end{array}$

C $1.84815-0.04498 \quad 0.59990$

N $1.937040 .98070-0.31766$

C $2.902311 .10935-1.44590$

C $2.513712 .40547-2.18855$

B $0.32594 \quad 1.66786 \quad 1.03488$

$\begin{array}{lll}\mathrm{Rh}-0.14931 & 0.28165 & -0.37264\end{array}$

$\begin{array}{llll}\mathrm{P} & -2.19835 & -0.75313 & 0.02321\end{array}$

C $-3.752130 .11280-0.69226$

C $-5.10034-0.33597-0.09526$

$\begin{array}{llll}\text { C } & 0.11918 & 3.19720 & 1.35318\end{array}$

C 1.498203 .763541 .79842 
$\begin{array}{llll}\text { C } & -0.40049 & 4.00340 & 0.14044\end{array}$

$\begin{array}{llll}\text { C } & -0.87731 & 3.34319 & 2.53632\end{array}$

$\mathrm{Cl}-0.77174 \quad 0.79480-2.61825$

C -2.17257 -2.49819-0.78745

C $-3.41311-3.37793-0.53119$

C $-2.66717-1.18769 \quad 1.82087$

C $-1.74034-2.26257 \quad 2.41947$

C $-1.85906-2.46193-2.29395$

$\begin{array}{llll}C & -2.71593 & 0.06561 & 2.71004\end{array}$

C $-3.61967 \quad 1.64617-0.63833$

C $4.32137 \quad 1.27220-0.84781$

C $2.86972-0.07497-2.43929$

H $-3.68950-1.604161 .75726$

H $-3.70718-0.18552-1.75660$

H - $1.30737-2.95701-0.27110$

H $0.59502-0.303252 .42072$

C $2.65705-1.303220 .70439$

H -1.97617 -2.40519 3.48974

H - $1.85341-3.240641 .92507$

H $-0.67993-1.970962 .34449$

H $-3.04276-0.207373 .73014$

H $-1.72376 \quad 0.537632 .78990$

$\begin{array}{llll}\mathrm{H} & -3.41753 & 0.82480 & 2.32724\end{array}$

H $-4.483722 .10997-1.14889$

H $-3.61202 \quad 2.019510 .40101$

H -2.70011 $1.97885-1.14200$

H $-5.923610 .12268-0.67266$

H -5.25193 - $1.42580-0.11424$

H -5.214460 .005020 .94859$

H - $1.74932-3.49674-2.66761$

H $-2.67481-1.98872-2.86729$

H $-0.94217-1.90020-2.52351$

H -4.27861 -3.02725 - 1.11807

H -3.20050 -4.41116-0.86134

$\begin{array}{llll}\mathrm{H} & -3.71306 & -3.42752 & 0.52827\end{array}$

$\begin{array}{lllll}\mathrm{H} & 1.39998 & 4.83887 & 2.04258\end{array}$

H 1.881723 .244942 .69431

H $2.25078 \quad 3.661860 .99901$

$\begin{array}{llll}\mathrm{H} & -0.95717 & 4.40870 & 2.82451\end{array}$

H - 1.888442 .996252 .26436

H $-0.548202 .78228 \quad 3.42932$

H $-0.48899 \quad 5.075240 .40274$

H $0.279573 .91480-0.72205$

H -1.39144 $3.64793-0.18503$

H $4.344322 .10131-0.12019$

H $4.659960 .35207-0.34461$

H $5.03891 \quad 1.50220-1.65431$

H $3.537980 .14034-3.29186$

H $3.21337-1.01103-1.97229$

H $1.84811-0.21407-2.82919$

H $3.207752 .58170-3.02835$

H $1.488982 .32643-2.58537$

H $2.567643 .27250-1.50826$

C $2.28628-2.469320 .00289$

C $3.01333-3.658620 .16703$

C $4.11731-3.697331 .03439$

C $4.48651-2.543201 .74422$

C $3.75738-1.354181 .58718$

H $1.42241-2.42871-0.66994$

H $2.71592-4.55653-0.38486$

H $4.68523-4.624941 .16012$

H $5.34200-2.567982 .42733$ 
H $4.04008-0.457522 .14883$

TS(3-D3)
SCF Energy $=-1191.71674710$
Enthalpy 0K $=-1191.080250$
Enthalpy 298K $=-1191.040268$
Free Energy 298K $=-1191.148436$
SCF $($ C6H6) $=-1191.72201106$
SCF(BP86-D3) $=-0.13593292$

Lowest Frequencies $=-123.648426 .0994 \mathrm{~cm}-1$

C $-3.365772 .37427-1.02036$

C $-3.26857 \quad 1.17463-0.27303$

$\begin{array}{llll}\text { C } & -4.39761 & 0.75864 & 0.47347\end{array}$

$\begin{array}{llll}C & -5.57734 & 1.51515 & 0.46998\end{array}$

C $-5.65894 \quad 2.70292-0.27674$

C $-4.54576 \quad 3.13038-1.01918$

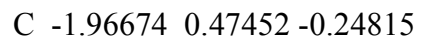

N $-1.76250-0.97591-0.16447$

B $-0.75290-0.894270 .92654$

C $-0.87440-1.234142 .48608$

C $0.50767-1.325113 .17118$

C $-0.72778 \quad 1.04388-0.05310$

Rh $0.60681-0.40636-0.46588$

Cl $1.64330-2.20793-1.67862$

$\begin{array}{lllll}\text { P } & 2.54677 & 0.67429 & 0.04998\end{array}$

C $2.71548 \quad 1.69829 \quad 1.67204$

C 1.439472 .424342 .12404

C $4.12319-0.399340 .28506$

C $4.84615-0.82728-1.00277$

C $2.96231 \quad 1.88350-1.37318$

C $2.112953 .16784-1.31734$

C $3.80974-1.617241 .17379$

C $-2.38709-2.02407-1.04289$

C $-2.20415-1.58604-2.51164$

C $-1.65962-3.35922-0.79454$

C $-3.89255-2.21419-0.74021$

C $-1.70307-0.108613 .16922$

C $-1.61596-2.578492 .70570$

$\begin{array}{llll}\text { C } & 2.76300 & 1.20701 & -2.74534\end{array}$

C 3.925642 .656861 .68996

$\begin{array}{lllll}\mathrm{H} & 2.91402 & 0.88960 & 2.40287\end{array}$

$\begin{array}{lllll}\mathrm{H} & 4.79921 & 0.27479 & 0.84518\end{array}$

H $4.030432 .14447-1.23823$

$\begin{array}{llll}\mathrm{H} & -0.62641 & 2.10304 & 0.21081\end{array}$

$\begin{array}{lllll}\text { H } & 3.29399 & 0.25085 & -2.84641\end{array}$

H $1.69244 \quad 0.99917-2.91799$

H $3.11222 \quad 1.88704-3.54458$

H $2.359743 .80784-2.18403$

H $\quad 1.03817 \quad 2.92561-1.38014$

H $2.279353 .76594-0.40822$

$\begin{array}{lllll}\mathrm{H} & 1.58682 & 2.83748 & 3.13855\end{array}$

$\begin{array}{lllll}\mathrm{H} & 1.18632 & 3.26774 & 1.46031\end{array}$

$\begin{array}{lllll}\mathrm{H} & 0.57602 & 1.74427 & 2.15167\end{array}$

$\begin{array}{llll}\text { H } & 4.87192 & 2.17420 & 1.39621\end{array}$

H $3.771373 .52479 \quad 1.02787$

H 4.064703 .049622 .71340

H $4.74867-2.15111 \quad 1.40894$

H $3.34043-1.333532 .13071$

H $3.13308-2.309840 .64815$

H $5.74833-1.40422-0.72706$

H $4.20298-1.47547-1.61892$

H $5.18102 \quad 0.03246-1.60603$ 
$\begin{array}{llll}\text { H } & -2.67201 & -2.32336 & -3.18605 \\ \text { H } & -2.67936 & -0.60699 & -2.69505 \\ \text { H } & -1.13181 & -1.51584 & -2.76125 \\ \text { H } & -4.27148 & -3.05972 & -1.33977 \\ \text { H } & -4.05894 & -2.45749 & 0.32321 \\ \text { H } & -4.33946 & -0.15323 & 1.07419 \\ \text { H } & -2.05487 & -4.11733 & -1.49168 \\ \text { H } & -0.57565 & -3.26386 & -0.97031 \\ \text { H } & -1.83605 & -3.72501 & 0.22954 \\ \text { H } & -1.82447 & -0.34186 & 4.24457 \\ \text { H } & -1.20924 & 0.87452 & 3.09307 \\ \text { H } & -2.71061 & -0.00978 & 2.73176 \\ \text { H } & -1.74408 & -2.76196 & 3.78955 \\ \text { H } & -2.61962 & -2.57574 & 2.24692 \\ \text { H } & -1.05037 & -3.43087 & 2.29262 \\ \text { H } & 0.38791 & -1.54133 & 4.25028 \\ \text { H } & 1.12203 & -2.12567 & 2.72731 \\ \text { H } & 1.06875 & -0.38089 & 3.07688 \\ \text { H } & -4.48639 & -1.32461 & -0.99768 \\ \text { H } & -6.43580 & 1.17917 & 1.06146 \\ \text { H } & -6.58371 & 3.28894 & -0.28083 \\ \text { H } & -4.59974 & 4.05115 & -1.60979 \\ \text { H } & -2.50340 & 2.69529 & -1.61423\end{array}$

TS(3-D4)

SCF Energy $=-1191.71565602$

Enthalpy $0 \mathrm{~K}=-1191.080159$

Enthalpy $298 \mathrm{~K}=-1191.039888$

Free Energy 298K $=-1191.149786$

$\mathrm{SCF}(\mathrm{C} 6 \mathrm{H} 6)=-1191.72012108$

$\mathrm{SCF}(\mathrm{BP} 86-\mathrm{D} 3)=-0.12954624$

Lowest Frequencies $=-132.922817 .5476 \mathrm{~cm}-1$

C $2.075874 .48687-1.25066$

C $1.82924 \quad 3.11702-1.39278$

C $1.087262 .41811-0.40311$

$\begin{array}{lllll}\text { C } & 0.62521 & 3.11842 & 0.74063\end{array}$

C 0.888994 .483850 .88370

C $1.608605 .17003-0.11305$

C $0.82392 \quad 1.00743-0.56698$

C $1.13069-0.01896-1.47680$

B $2.19583-0.93580-0.71916$

C $3.22624-1.87885-1.53913$

C $4.39210-0.99031-2.05634$

Rh $-0.16600-0.396310 .22651$

Cl $-0.97304-1.589632 .14024$

$\begin{array}{llll}\text { N } & 1.93996 & -0.57719 & 0.64893\end{array}$

C $2.63470-0.75007 \quad 1.93664$

C $2.53261-2.201122 .47208$

P $-2.47132-0.19831-0.23590$

C $-3.30402-1.91109-0.41607$

C $-2.38649-2.93345-1.11312$

C $4.12016-0.34903 \quad 1.75194$

$\begin{array}{llll}\text { C } & 2.01379 & 0.21457 & 2.97640\end{array}$

C $3.81526-3.08149-0.76593$

C $2.48143-2.45584-2.77513$

$\begin{array}{llll}\text { C } & -3.56009 & 0.70715 & 1.07212\end{array}$

$\begin{array}{llll}\text { C } & -2.73680 & 1.77529 & 1.81544\end{array}$

C $-2.993660 .74143-1.81688$

C $-2.632412 .23563-1.73971$

C $-4.26864-0.21002 \quad 2.08705$

C $-2.402450 .10565-3.08699$

C $-4.70890-1.87583-1.04475$ 


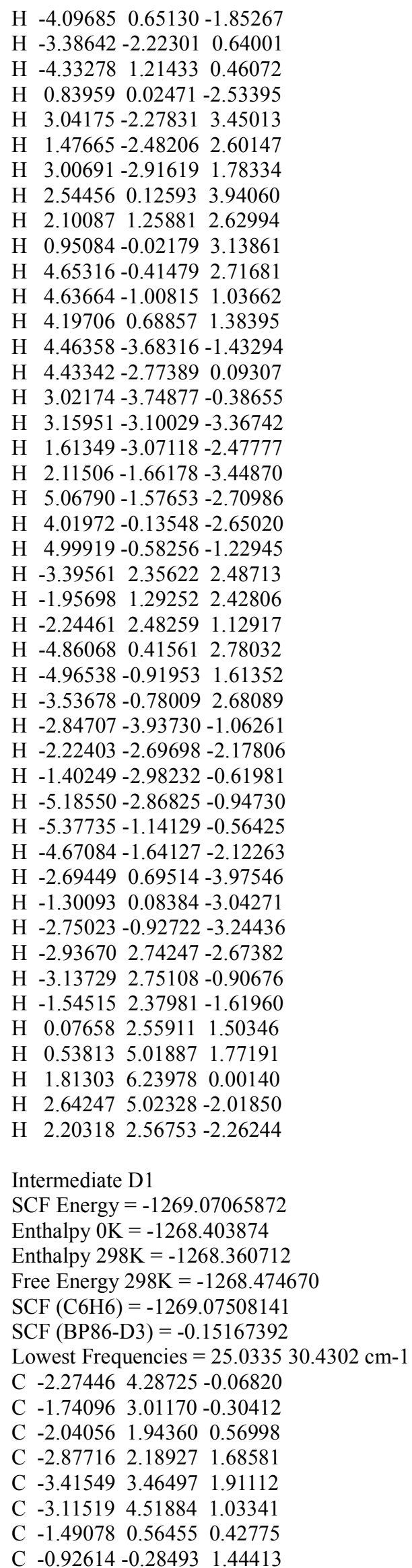


Rh $0.19500-0.38090-0.50160$ Cl $0.52859 \quad 1.35568-2.29457$ N -2.00139 $-0.57155-0.39008$ C $-3.03435-0.58870-1.47598$

C $-2.67753-1.71406-2.46818$

C $-4.41537-0.85189-0.83050$ C $-3.077340 .74190-2.25277$

B $-1.52050-1.524350 .73912$

C $-1.91493-2.981301 .28802$

C $-2.25977-4.07558 \quad 0.25251$

C $-0.77792-3.49970 \quad 2.20795$

C $-3.15899-2.769302 .20588$

$\begin{array}{lllll}\text { P } & 2.40724 & 0.17042 & 0.11023\end{array}$

$\begin{array}{llll}\text { C } & 2.58778 & 1.98484 & 0.68695\end{array}$

$\begin{array}{llll}\text { C } & 3.92930 & 2.38708 & 1.33242\end{array}$

C $0.71608-2.48625-0.84865$

C $0.78608-1.85032-1.94491$

C $3.17977-1.002461 .40913$

C $4.68016-0.80798 \quad 1.71836$

C $3.53752-0.00988-1.44477$

C $4.65751 \quad 1.03088-1.63533$

C $2.37066-1.068662 .71565$

C $4.09533-1.43542-1.63638$

C $1.430032 .41792 \quad 1.60211$

H $3.05799-1.973230 .89041$

H $2.781120 .17992-2.22730$

H $2.463922 .51431-0.27692$

H $-0.455640 .04926 \quad 2.36760$

H $0.97669-1.72835-3.00397$

H $\quad 0.85356-3.36753-0.23569$

H $-3.44654-3.728302 .67894$

H -2.94726 -2.04745 3.01389

H $-4.03539-2.39956 \quad 1.64761$

H - $1.09232-4.431452 .71586$

H $\quad 0.14952-3.72490 \quad 1.65529$

H $-0.52638-2.762962 .99036$

H -2.45915 -5.03700 0.76409

H -3.16620 -3.82647 - 0.32538

H $-1.44026-4.24441-0.46699$

H -3.50393 - $1.84162-3.18859$

H - $1.76442-1.45474-3.02649$

H $-2.51104-2.67539-1.96136$

H -5.20307-0.81020-1.60246

H -4.45697-1.84629-0.35715

H $-4.64438-0.08801-0.06836$

H $-3.729640 .60628-3.13263$

H $-3.49391 \quad 1.56235-1.64997$

H $-2.07179 \quad 1.02618-2.60303$

H $1.514603 .50007 \quad 1.81055$

H $0.453592 .23919 \quad 1.13476$

H $1.45794 \quad 1.894322 .57372$

H $3.985523 .49002 \quad 1.38174$

H 3.995372 .017262 .36967

H $4.814612 .03457 \quad 0.78637$

H $2.78485-1.857703 .36998$

H $2.42767-0.119353 .27587$

H $1.31279-1.302522 .52804$

H $5.07584-1.72382 \quad 2.19468$

H $5.29031-0.60950 \quad 0.82449$

H 4.842390 .021072 .42462

H $4.90363-1.67241-0.92451$

H $3.31634-2.20699-1.54870$ 
H $4.52732-1.51366-2.65109$

H $5.073370 .91468-2.65326$

H $4.298222 .06803-1.55048$

H $5.494090 .88779-0.92910$

H $-3.10559 \quad 1.36727 \quad 2.37270$

H $-4.06548 \quad 3.63492 \quad 2.77617$

H -3.530035 .516941 .21052$

H -2.02654 $5.10745-0.75068$

H $-1.072402 .82397-1.15080$

Intermediate D2

SCF Energy $=-1191.73708145$

Enthalpy 0K $=-1191.099423$

Enthalpy $298 \mathrm{~K}=-1191.058750$

Free Energy 298K = -1191.169044

$\mathrm{SCF}(\mathrm{C} 6 \mathrm{H} 6)=-1191.74179117$

$\mathrm{SCF}(\mathrm{BP} 86-\mathrm{D} 3)=-0.12874801$

Lowest Frequencies $=21.782726 .8208 \mathrm{~cm}-1$

C $-1.047340 .74458-1.00055$

C $-2.128780 .11596-0.24725$

$\begin{array}{llll}\mathrm{N} & -1.77933 & -0.03956 & 1.02621\end{array}$

$\begin{array}{llll}\text { C } & -2.59727 & -0.38801 & 2.22118\end{array}$

$\begin{array}{llll}\text { C } & -2.07120 & 0.51413 & 3.36447\end{array}$

$\begin{array}{llll}\text { B } & -0.11029 & 1.70919 & -0.25055\end{array}$

Rh $0.29845-0.04370 \quad 0.53290$

P $2.33393-0.57870-0.49580$

C $3.86880-0.598950 .63897$

C $5.17883-1.05271-0.02937$

C $0.118613 .26409-0.07101$

$\begin{array}{llll}\text { C } & -1.18125 & 3.77620 & 0.62219\end{array}$

C $1.32556 \quad 3.62278 \quad 0.82427$

C $0.237483 .96507-1.45033$

Cl $1.12242-0.909392 .60676$

C $2.07605-2.42045-1.03128$

C $2.92100-2.94471-2.21021$

C $2.911660 .21997-2.12783$

$\begin{array}{llll}\text { C } & 1.77466 & 0.24920 & -3.16469\end{array}$

C $2.17814-3.37690 \quad 0.17339$

C $3.517911 .62174-1.95786$

$\begin{array}{llll}C & 4.04078 & 0.72516 & 1.40817\end{array}$

C $-4.10301-0.096132 .02739$

C $-2.37804-1.872242 .59531$

H $3.70666-0.45689-2.49098$

H $3.56194-1.354741 .38360$

H $1.01789-2.39755-1.35534$

H $-1.027640 .56496-2.08280$

C $-3.35545-0.33922-0.96986$

$\begin{array}{llll}\text { H } & 2.17318 & 0.54338 & -4.15274\end{array}$

H $1.26872-0.72338-3.28059$

H $1.009440 .98877-2.88016$

H $3.766182 .04218-2.94980$

H $2.807622 .31197-1.47402$

H $4.442591 .61380-1.36115$

H 4.804340 .590042 .19600

H $4.38072 \quad 1.549380 .75933$

H $3.100881 .02219 \quad 1.89714$

H $5.98219-1.093850 .72893$

H $5.10053-2.05585-0.47770$

H $5.51522-0.35511-0.81622$

H $1.78970-4.37130-0.11230$

H $3.22761-3.51522 \quad 0.48773$

H $1.60881-3.016301 .04519$ 
H $3.99925-2.95983-1.97960$

H $2.62369-3.98773-2.42434$

H $2.77941-2.37113-3.13980$

H $-1.11365 \quad 4.87112 \quad 0.77164$

$\begin{array}{llll}\mathrm{H} & -2.07827 & 3.57260 & 0.01223\end{array}$

H -1.325153 .310001 .61160$

H $0.280495 .06289-1.31501$

H $\quad 1.150653 .66501-1.99267$

H $-0.629053 .73892-2.09609$

$\begin{array}{lllll}\mathrm{H} & 1.38219 & 4.71734 & 0.98010\end{array}$

H $1.24375 \quad 3.13723 \quad 1.81117$

$\begin{array}{lllll}\text { H } & 2.28011 & 3.29725 & 0.37812\end{array}$

H $-4.26614 \quad 0.92717 \quad 1.64786$

H $-4.59703-0.801951 .34405$

H - $4.60075-0.173443 .00914$

H -2.90978 -2.09969 3.53572

H $-2.77105-2.544261 .81358$

H $-1.30535-2.075562 .73927$

H $-2.58358 \quad 0.261024 .30893$

H -0.987330 .374563 .49540$

H $-2.27110 \quad 1.57571 \quad 3.13802$

C $-3.79727-1.67980-0.92043$

C $-4.89640-2.09463-1.68680$

C $-5.57221-1.17680-2.50806$

$\begin{array}{llll}\text { C } & -5.13276 & 0.15591 & -2.57354\end{array}$

C $-4.023160 .56790-1.82180$

H -3.26207 -2.39767-0.29144

H $-5.22393-3.13868-1.64458$

H $-6.43384-1.50112-3.10078$

H $-5.652790 .87504-3.21510$

H $-3.670801 .60346-1.87736$

Intermediate D3

SCF Energy $=-1191.71965333$

Enthalpy $0 \mathrm{~K}=-1191.082522$

Enthalpy $298 \mathrm{~K}=-1191.041950$

Free Energy 298K $=-1191.152373$

$\mathrm{SCF}(\mathrm{C} 6 \mathrm{H} 6)=-1191.72399101$

$\mathrm{SCF}(\mathrm{BP} 86-\mathrm{D} 3)=-0.13424826$

Lowest Frequencies $=26.020528 .2999 \mathrm{~cm}-1$

C $-3.506942 .41984-0.82481$

C $-3.21427 \quad 1.25859-0.06962$

$\begin{array}{llll}C & -4.14518 & 0.84951 & 0.91644\end{array}$

C $-5.32386 \quad 1.57434 \quad 1.13611$

$\begin{array}{llll}C & -5.59979 & 2.72702 & 0.38006\end{array}$

C $-4.68388 \quad 3.14758-0.59839$

C $-1.911950 .57909-0.24696$

N $-1.76181-0.89076-0.18736$

B $-0.62121-1.247140 .68410$

C $-0.52249-1.995852 .08969$

C $\quad 0.87561-2.59715 \quad 2.34822$

C $-0.68356 \quad 1.17114-0.28476$

Rh $0.58403-0.33292-0.59833$

Cl $1.55669-2.23721-1.74817$

$\begin{array}{lllll}\text { P } & 2.45665 & 0.78424 & 0.06830\end{array}$

C 2.482881 .350081 .90099

$\begin{array}{llll}\text { C } & 1.27757 & 2.21760 & 2.31005\end{array}$

C $4.13307-0.12743-0.06218$

C $4.55892-0.43390-1.50974$

C $2.79807 \quad 2.29918-1.06439$

C $2.26733 \quad 3.65659-0.57245$

C $4.15290-1.403920 .79600$ 
C $-2.52937-1.78673-1.15007$

C $-2.40837-1.17814-2.56325$

C $-1.89110-3.18899-1.14841$

C $-4.01902-1.91579-0.75684$

$\begin{array}{llll}\text { C } & -0.79401 & -0.87048 & 3.13818\end{array}$

C $-1.58912-3.098642 .30628$

C $2.265712 .00271-2.48317$

$\begin{array}{llll}\text { C } & 3.80903 & 1.98221 & 2.37105\end{array}$

$\begin{array}{llll}\mathrm{H} & 2.36783 & 0.37337 & 2.40984\end{array}$

$\begin{array}{lllll}\mathrm{H} & 4.84994 & 0.60472 & 0.35885\end{array}$

H $3.903372 .35695-1.10348$

H $-0.567512 .25541-0.16675$

H $2.601891 .03027-2.87521$

H $1.161681 .99526-2.48824$

H $2.604232 .79013-3.18180$

H $2.51804 \quad 4.43262-1.31888$

H $1.170743 .65358-0.46238$

$\begin{array}{lllll}\mathrm{H} & 2.71042 & 3.96765 & 0.38621\end{array}$

H $1.21187 \quad 2.252703 .41265$

H $1.37847 \quad 3.25619 \quad 1.95840$

H $\quad 0.32910 \quad 1.81540 \quad 1.92223$

H 4.661651 .288482 .30396

H $4.063852 .89248 \quad 1.80110$

H $3.714302 .27978 \quad 3.43113$

H $5.14993-1.87607 \quad 0.73044$

H $3.95470-1.204281 .86305$

H $3.40715-2.125920 .42552$

H $5.53458-0.95325-1.48968$

H $3.82806-1.09627-2.00138$

H $4.690780 .47645-2.11729$

H -2.94626-1.81329-3.28745

H - $2.84236-0.16556-2.60591$

H - $1.34879-1.12319-2.86817$

H -4.49755 -2.65354-1.42422

$\begin{array}{llll}\mathrm{H} & -4.12947 & -2.28021 & 0.27835\end{array}$

H $-3.92838-0.038421 .51911$

H -2.36889 -3.79682 -1.93554

H $-0.81005-3.14325-1.36434$

H $-2.05540-3.70709-0.19178$

H $-0.77743-1.311234 .15355$

H $-0.02742-0.080703 .10068$

H $-1.78029-0.394622 .99615$

H - $1.58145-3.415043 .36681$

H -2.60881 -2.74826 2.07064

H -1.38205 -3.99545 1.69972

H $\quad 0.92325-3.04068 \quad 3.36144$

H $1.11617-3.38421 \quad 1.61429$

H $1.66158-1.828942 .27245$

H -4.56421 -0.96596 -0.85404

H $-6.02749 \quad 1.24322 \quad 1.90764$

H $-6.52238 \quad 3.29109 \quad 0.55151$

H $-4.89257 \quad 4.04027-1.19792$

H -2.80000 2.73705 -1.59907

Intermediate D4

SCF Energy $=-1191.73071032$

Enthalpy 0K $=-1191.094100$

Enthalpy $298 \mathrm{~K}=-1191.053583$

Free Energy 298K = -1191.163248

$\mathrm{SCF}(\mathrm{C} 6 \mathrm{H6})=-1191.73495254$

$\mathrm{SCF}(\mathrm{BP} 86-\mathrm{D} 3)=-0.13124061$

Lowest Frequencies $=24.628728 .7527 \mathrm{~cm}-1$ 
$\begin{array}{llll}C & -1.14053 & 4.83293 & 1.76631\end{array}$

$\begin{array}{llll}\text { C } & -1.11494 & 3.43627 & 1.80980\end{array}$

$\begin{array}{llll}\text { C } & -0.56482 & 2.69456 & 0.72537\end{array}$

C $-0.079163 .39442-0.41195$

C $-0.138284 .79051-0.45713$

$\begin{array}{llll}C & -0.65527 & 5.51403 & 0.63372\end{array}$

$\begin{array}{llll}C & -0.53992 & 1.25935 & 0.78257\end{array}$

$\begin{array}{llll}\text { C } & -1.10033 & 0.21592 & 1.50182\end{array}$

$\begin{array}{llll}\text { B }-2.08472 & -0.80717 & 0.71322\end{array}$

$\begin{array}{llll}\text { C } & -2.83489 & -1.97762 & 1.56874\end{array}$

C -4.18243 -1.43364 2.11473

Rh $-0.05677-0.17815-0.38922$

Cl $0.72269-0.09466-2.65325$

N $-2.03950-0.51558-0.67261$

C $-2.90825-0.43077-1.85329$

C -2.60207-1.58861 - 2.83524

$\begin{array}{llll}\text { P } & 2.26150 & -0.59511 & 0.17172\end{array}$

C $2.67529-2.32969-0.50916$

C $1.48639-3.30530-0.40806$

C $-4.39415-0.48592-1.42446$

C $-2.670730 .93036-2.55603$

C $-3.09102 \quad-3.237190 .70573$

C $-1.98340-2.424832 .78222$

C $3.665930 .51970-0.53142$

C $3.165831 .96700-0.69208$

C $2.76896-0.652202 .01082$

$\begin{array}{llll}\text { C } & 2.69127 & 0.74442 & 2.65568\end{array}$

$\begin{array}{llll}\text { C } & 4.28881 & 0.03435 & -1.85459\end{array}$

C $1.94928-1.676012 .81336$

C $3.96833-2.947020 .05411$

H $3.82921-0.973952 .00040$

H $2.81681-2.10574-1.58189$

$\begin{array}{lllll}\mathrm{H} & 4.44400 & 0.49041 & 0.25674\end{array}$

H $-0.84194 \quad 0.030592 .55368$

H -3.24180 - $1.51229-3.73259$

H $-1.54796-1.54844-3.15158$

H $-2.79285-2.56383-2.35624$

H $-3.319901 .01814-3.44542$

H $-2.90803 \quad 1.76011-1.86820$

H -1.62068 $1.02009-2.87180$

H -5.04056 -0.34131 -2.30760

H $-4.65955-1.45435-0.97196$

H $-4.620010 .31468-0.69944$

H $-3.59585-4.021821 .30296$

H $-3.72852-3.02726-0.16806$

H $-2.14509-3.662250 .32568$

H -2.49174 $-3.24277 \quad 3.32866$

H $-0.99432-2.803722 .46859$

H - $1.82215-1.608633 .50917$

H -4.69037-2.20212 2.73028

H -4.02877-0.54551 2.75430

H -4.87466-1.14515 1.30665

H $4.014272 .63359-0.93234$

H $2.441702 .01605-1.52081$

$\begin{array}{llll}\mathrm{H} & 2.67132 & 2.35773 & 0.21149\end{array}$

H $5.06303 \quad 0.75799-2.17014$

H $4.77918-0.94830-1.76907$

H $3.52881-0.01685-2.65076$

H $1.73116-4.24226-0.94126$

H $1.24520-3.570540 .63472$

H $0.57584-2.88710-0.87031$

H $4.22958-3.85487-0.51943$ 
H $4.83109-2.26215-0.00139$

H $3.84963-3.253921 .10733$

H $2.25249-1.651393 .87616$

H $0.87180-1.452572 .76262$

H $2.09337-2.706842 .45246$

H $2.97744 \quad 0.68005 \quad 3.72126$

H $3.36786 \quad 1.469732 .17579$

H 1.667091 .151372 .60500

H $0.309732 .81156-1.25240$

H $\quad 0.221315 .32078-1.34487$

$\begin{array}{llll}\mathrm{H} & -0.68963 & 6.60785 & 0.59808\end{array}$

H -1.555345 .396042 .60876$

H - 1.514962 .896782 .67428

\section{TS(D1-E1)}

SCF Energy $=-1269.06387153$

Enthalpy $0 \mathrm{~K}=-1268.397869$

Enthalpy $298 \mathrm{~K}=-1268.355630$

Free Energy 298K = -1268.467411

$\mathrm{SCF}(\mathrm{C} 6 \mathrm{H} 6)=-1269.06801197$

$\mathrm{SCF}(\mathrm{BP} 86-\mathrm{D} 3)=-0.15135303$

Lowest Frequencies $=-203.018727 .8746 \mathrm{~cm}-1$

C $-2.61335 \quad 4.139920 .01358$

C $-1.998692 .90175-0.22508$

C $-2.29169 \quad 1.79383 \quad 0.60204$

C $-3.199681 .96127 \quad 1.67468$

C -3.817363 .200451 .90199$

$\begin{array}{llll}C & -3.52680 & 4.29335 & 1.07007\end{array}$

C -1.646720 .461050 .45378$

C $-0.90173-0.31229 \quad 1.37857$

$\mathrm{Rh} 0.28047-0.27176-0.52467$

Cl $0.58988 \quad 1.50116-2.18544$

N $-1.89398-0.61803-0.49545$

C $-2.89799-0.67876-1.60666$

C $-2.50352-1.82899-2.55324$

C $-4.29943-0.93816-1.00341$

$\begin{array}{llll}C & -2.93303 & 0.62927 & -2.42478\end{array}$

B -1.23151 -1.641220 .56438$

C $-1.82534-2.98075 \quad 1.28430$

C $-2.28754-4.127300 .35695$

C $-0.76744-3.53851 \quad 2.27214$

C -3.04061 -2.53293 2.14683

$\begin{array}{llll}\text { P } & 2.47772 & 0.23780 & 0.10369\end{array}$

C 2.662432 .052740 .67992

C 3.962962 .421081 .41925

C $0.33692-2.48506-0.61508$

C $0.73235-1.88744-1.70328$

C $3.20980-0.950211 .41016$

C $4.71844-0.82157 \quad 1.70998$

C $3.611780 .05982-1.43503$

C $4.813331 .01612-1.55593$

C $2.40080-0.951292 .71922$

C $4.04590-1.39913-1.68057$

C $1.44018 \quad 2.51742 \quad 1.49052$

H $3.03973-1.923570 .90975$

H $2.883340 .34291-2.21666$

H $2.629992 .57843-0.29327$

H $-0.51392 \quad 0.03605 \quad 2.33573$

H $1.10495-2.04767-2.71382$

H $0.50407-3.44429-0.12422$

H -3.47313 -3.40245 2.67848

H - $2.74028-1.790302 .90604$ 


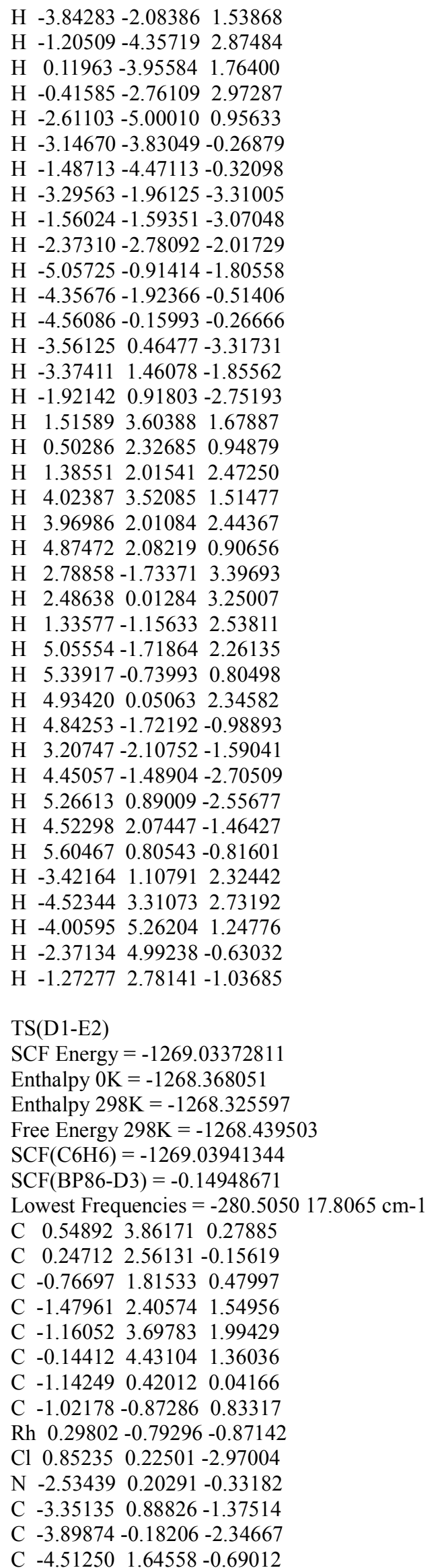


C $-2.49370 \quad 1.88587-2.17947$

$\begin{array}{llll}\text { B } & -2.62459 & -0.90273 & 0.59030\end{array}$

C $-3.77577-1.61942 \quad 1.43757$

C $-5.04727-1.984020 .63389$

C $-3.24054-2.911052 .10600$

C $-4.16636-0.619012 .56765$

$\begin{array}{llll}\text { P } & 2.50564 & -0.26647 & 0.20526\end{array}$

$\begin{array}{llll}\text { C } & 2.76771 & 0.76097 & 1.81334\end{array}$

$\begin{array}{llll}C & 4.10585 & 0.58916 & 2.56061\end{array}$

C $-0.88318-2.46091-0.31906$

C $-0.98794-2.16967-1.60176$

C $3.23787-2.016480 .50906$

C $4.74793-2.152290 .79505$

C $3.668290 .54805-1.09331$

C $4.87902 \quad 1.36088-0.60260$

C $2.42184-2.788261 .56472$

C $4.08367-0.45429-2.18991$

$\begin{array}{lllll}\text { C } & 1.60888 & 0.58177 & 2.81345\end{array}$

H $3.03631-2.48696-0.47368$

H $2.957311 .24588-1.57476$

H $2.70721 \quad 1.79541 \quad 1.42228$

H - $0.50750-0.957271 .79456$

H - $1.38595-2.51727-2.55248$

$\begin{array}{llll}\mathrm{H} & -0.94628 & -3.28388 & 0.39308\end{array}$

H $-4.93259-1.071843 .22592$

H -3.30066 - 0.359253 .20354

H $-4.58355 \quad 0.318142 .16126$

H $-3.99402-3.323612 .80328$

H -3.02142 -3.69441 1.35990

H -2.31906-2.72503 2.68538

H $-5.78904-2.46845 \quad 1.29767$

H $-5.53459-1.099120 .19204$

H $-4.82326-2.69178-0.18318$

H $-4.54833 \quad 0.29274-3.10217$

H -3.06719-0.68054 -2.86959

H $-4.49111-0.94644-1.81955$

H $-5.129212 .15870-1.44817$

H $-5.170660 .96322-0.12661$

$\begin{array}{llll}\mathrm{H} & -4.12012 & 2.40482 & 0.00683\end{array}$

H $-3.096512 .26677-3.02133$

H -2.18651 2.74921 -1.56964

H - $-1.591751 .40217-2.59075$

H $1.77157 \quad 1.23985 \quad 3.68696$

H $\quad 0.63763 \quad 0.85005 \quad 2.37646$

H $1.55456-0.453323 .19313$

H 4.206291 .390653 .31638

H $4.12997-0.368973 .10669$

$\begin{array}{lllll}\mathrm{H} & 4.98893 & 0.63250 & 1.90893\end{array}$

H $2.71677-3.853621 .56612$

H $2.60588-2.398572 .58113$

H $1.34032-2.73492 \quad 1.36063$

H $5.04924-3.208730 .66746$

H $5.37312-1.548630 .12039$

H $4.99556-1.86703 \quad 1.82910$

H $4.85309-1.16335-1.84074$

H $3.21973-1.01360-2.57972$

H $4.51124 \quad 0.10404-3.04224$

H $5.32617 \quad 1.88907-1.46540$

$\begin{array}{llll}\mathrm{H} & 4.61119 & 2.12701 & 0.14308\end{array}$

H $5.670880 .72376-0.17228$

H -2.28413 1.842932 .03532

H - 1.712884 .133772 .83376 
H $\quad 0.09908 \quad 5.44299 \quad 1.70089$

H $1.325604 .43567-0.23811$

H $0.761422 .11600-1.01479$

\section{TS(D1-E3)}

SCF Energy $=-1269.02444385$

Enthalpy 0K $=-1268.360098$

Enthalpy $298 \mathrm{~K}=-1268.316899$

Free Energy $298 \mathrm{~K}=-1268.432833$

$\mathrm{SCF}(\mathrm{C} 6 \mathrm{H6})=-1269.03346260$

$\mathrm{SCF}(\mathrm{BP} 86-\mathrm{D} 3)=-0.14229815$

Lowest Frequencies $=-286.654021 .7398 \mathrm{~cm}-1$

C $2.72647 \quad 1.84457 \quad 0.32884$

$\begin{array}{llll}\text { C } & 2.13010 & 0.51374 & 0.07195\end{array}$

C $\quad 1.07140 \quad 0.28378-2.13081$

C $0.127051 .17671-2.01186$

Rh $-0.62933-0.24966-0.90163$

$\begin{array}{llll}\text { P } & -2.69898 & 0.26301 & 0.20180\end{array}$

C $-3.50858 \quad 1.54844-0.97254$

C $-2.829802 .92488-0.83415$

B $1.64477-1.33890 \quad 0.70472$

N $2.81195-0.69743 \quad 0.03075$

C $4.06740-1.08784-0.69278$

C $3.72075-2.29422-1.59711$

C $1.38156-2.77702 \quad 1.34142$

$\begin{array}{llll}\text { C } & 0.80347 & 0.03119 & 0.59547\end{array}$

$\mathrm{Cl}-2.02614-1.10163-2.74754$

C $4.600370 .06125-1.57609$

C $5.16543-1.493290 .31830$

C $-3.96598-1.17437 \quad 0.26222$

C $-3.24668-2.50843 \quad 0.53276$

C $-2.62720 \quad 1.12911 \quad 1.91288$

$\begin{array}{llll}\text { C } & -2.11702 & 0.17568 & 3.00808\end{array}$

C $-5.19285-1.01745 \quad 1.17873$

C $-3.87543 \quad 1.88853 \quad 2.40463$

C $-5.04316 \quad 1.67226-0.97721$

H $-1.829781 .87542 \quad 1.72399$

H -3.20821 $1.10963-1.94239$

H $-4.29768-1.19897-0.79307$

H $\quad 0.44285 \quad 0.58731 \quad 1.47718$

H $-0.29140 \quad 2.12205-2.36466$

H $1.83920-0.06040-2.81989$

H $5.43651-0.32568-2.18178$

H $3.830370 .44457-2.26296$

H $4.978940 .90652-0.98274$

H $\quad 6.04878-1.86553-0.22826$

H $5.49001-0.63468 \quad 0.92895$

H $4.82316-2.288590 .99592$

H $4.60623-2.58685-2.18604$

H $3.41017-3.16637-1.00107$

H $2.90266-2.04785-2.29241$

H $-3.96700-3.344870 .46572$

H $-2.46004-2.68383-0.21838$

H $-2.79667-2.540751 .54083$

H $-5.89141-1.856811 .00257$

H $-4.91161-1.054882 .24545$

H $-5.74806-0.083531 .00452$

H $-1.93766 \quad 0.73505 \quad 3.94505$

H -2.85343 $-0.61413 \quad 3.23648$

H $-1.17396-0.312542 .72258$

H $-3.622912 .44791 \quad 3.32529$

H $-4.250332 .61788 \quad 1.67126$ 
$\begin{array}{llll}\mathrm{H} & -4.70150 & 1.20551 & 2.65559\end{array}$

H -3.158353 .466120 .07006$

H - $1.732272 .83206-0.78936$

H $-3.085273 .55901-1.70263$

H $-5.344332 .36917-1.78172$

H $-5.53616 \quad 0.70962-1.18615$

H $-5.449032 .07192-0.03263$

C $2.62537-3.54975 \quad 1.83881$

C $0.39873-2.64002 \quad 2.53081$

C $0.67595-3.61278 \quad 0.22851$

H $\quad 0.16053-3.637252 .94737$

H $0.82087-2.029093 .34858$

H $-0.54788-2.176952 .21156$

H $2.31642-4.512702 .28795$

H $3.32921-3.789721 .02457$

H $3.17657-2.989232 .61474$

$\begin{array}{llll}\mathrm{H} & 0.33467 & -4.57801 & 0.65071\end{array}$

H $-0.20845-3.09345-0.18173$

H $1.34558-3.83400-0.61879$

C $3.919821 .97105 \quad 1.08081$

C 4.410193 .234221 .43929

C $3.71876 \quad 4.39382 \quad 1.05361$

C $2.52146 \quad 4.281220 .32458$

C $2.02593 \quad 3.02055-0.02695$

$\begin{array}{lllll}\mathrm{H} & 4.43438 & 1.06814 & 1.42133\end{array}$

H 5.330563 .311702 .02733

H 4.106965 .381051 .32449

$\begin{array}{llll}\mathrm{H} & 1.97451 & 5.18131 & 0.02579\end{array}$

H $1.104522 .92344-0.60686$

TS(D1-E4)

SCF Energy $=-1269.04926848$

Enthalpy $0 \mathrm{~K}=-1268.384219$

Enthalpy $298 \mathrm{~K}=-1268.341053$

Free Energy $298 \mathrm{~K}=-1268.455826$

SCF $(\mathrm{C} 6 \mathrm{H} 6)=-1269.05514255$

$\mathrm{SCF}(\mathrm{BP} 86-\mathrm{D} 3)=-0.15126383$

Lowest Frequencies $=-154.276018 .9960 \mathrm{~cm}-1$

C $4.49368-2.964661 .00271$

C $3.42410-2.219940 .48205$

C $3.20625-0.899980 .93188$

C $4.04668-0.354541 .92701$

C $5.11989-1.102712 .43254$

C $5.34861-2.408491 .96830$

C $2.04079-0.099110 .44689$

$\begin{array}{llll}C & 0.88572 & 0.19892 & 1.23930\end{array}$

$\mathrm{Rh}-0.38188-0.01211-0.56993$

Cl $0.22495-2.49094-0.89228$

N $1.85966 \quad 0.53658-0.73677$

C $2.802550 .61790-1.89429$

C $2.350621 .81576-2.75797$

C $4.259220 .86929-1.43381$

C $2.73303-0.67503-2.73739$

$\begin{array}{lllll}\text { B } & 0.49483 & 1.51426 & 0.49707\end{array}$

$\begin{array}{lllll}\text { C } & 0.69519 & 3.07431 & 0.77068\end{array}$

C $0.16983 \quad 3.99387-0.35763$

C $-0.05775 \quad 3.442262 .08073$

$\begin{array}{llll}\text { C } & 2.20062 & 3.37843 & 0.99928\end{array}$

$\begin{array}{llll}\mathrm{P}-2.53500 & -0.48342 & 0.25972\end{array}$

C $-2.76050-0.519442 .16848$

C $-4.21649-0.596302 .67990$

C $-1.09718 \quad 1.31435-2.07212$ 
C $-0.962920 .16860-2.61327$

$\begin{array}{llll}C & -3.85219 & 0.68859 & -0.47663\end{array}$

$\begin{array}{llll}\text { C } & -5.32571 & 0.24158 & -0.41288\end{array}$

C $-2.99233-2.26269-0.28907$

C $-4.05544-3.04930 \quad 0.49830$

$\begin{array}{llll}\text { C } & -3.68952 & 2.12202 & 0.06153\end{array}$

C $-3.25947-2.32593-1.80402$

$\begin{array}{llll}\text { C } & -2.06119 & 0.63378 & 2.90704\end{array}$

H $-3.542250 .69112-1.53822$

H $-2.01223-2.75243-0.13161$

H -2.23649-1.46246 2.42163

H $\quad 0.72230-0.246802 .22008$

H $-0.96612-0.52332-3.44709$

H -1.34717 $2.36752-2.11051$

H $2.33458 \quad 4.44189 \quad 1.27579$

$\begin{array}{llll}\mathrm{H} & 2.61711 & 2.76429 & 1.81651\end{array}$

H $2.79796 \quad 3.188680 .09275$

H $0.11214 \quad 4.509732 .31859$

H -1.14634 3.293791 .98634

$\begin{array}{lllll}\text { H } & 0.29552 & 2.84741 & 2.94053\end{array}$

H $0.37735 \quad 5.05405-0.11602$

H $0.645073 .76977-1.32643$

H $-0.922623 .89583-0.48206$

H $2.98574 \quad 1.88422-3.65745$

H $1.303241 .69893-3.07382$

H $2.447662 .76316-2.20143$

H $4.877231 .08401-2.32277$

H $4.32358 \quad 1.73987-0.75938$

H $4.69382-0.00100-0.92173$

H $3.33947-0.55055-3.65200$

H $3.12190-1.53830-2.17679$

H $1.69456-0.90507-3.01971$

H $-2.05700 \quad 0.42571 \quad 3.99285$

H -1.025320 .781652 .58511$

H $-2.60094 \quad 1.584002 .76123$

H -4.20356 -0.834893 .75943$

H $-4.71687 \quad 0.381202 .57576$

$\mathrm{H}-4.83507-1.352112 .18041$

H $-4.26858 \quad 2.82845-0.56088$

$\mathrm{H}-4.069122 .211611 .09402$

$\begin{array}{llll}\mathrm{H} & -2.63485 & 2.44241 & 0.05634\end{array}$

H $-5.935480 .93005-1.02721$

H $-5.48218-0.77331-0.80795$

$\begin{array}{llll}\mathrm{H} & -5.73010 & 0.27509 & 0.61033\end{array}$

H -4.21482 - $1.85508-2.09414$

H - $2.44398-1.85498-2.37059$

H $-3.30315-3.38479-2.11526$

H $-4.09163-4.07508 \quad 0.08691$

H $-3.81101-3.145161 .56792$

$\begin{array}{llll}\mathrm{H} & -5.07208 & -2.63021 & 0.40608\end{array}$

$\begin{array}{lllll}\text { H } & 3.86686 & 0.66298 & 2.28985\end{array}$

H $5.77526-0.665243 .19308$

H $6.18430-2.993772 .36628$

H $4.65272-3.990810 .65445$

H $2.71755-2.65661-0.23257$

TS(D1-E5)

SCF Energy $=-1269.03543185$

Enthalpy $0 \mathrm{~K}=-1268.371511$

Enthalpy $298 \mathrm{~K}=-1268.328396$

Free Energy 298K = -1268.442711

$\mathrm{SCF}(\mathrm{C} 6 \mathrm{H} 6)=-1269.04059196$ 
$\mathrm{SCF}(\mathrm{BP} 86-\mathrm{D} 3)=-0.14865814$

Lowest Frequencies $=-76.305722 .4934 \mathrm{~cm}-1$

C $-1.027734 .45588-0.91271$

C $-0.705913 .09615-0.84082$

C $-1.06828 \quad 2.34208 \quad 0.30822$

C $-1.757642 .98686 \quad 1.37448$

C $-2.06494 \quad 4.34775 \quad 1.29543$

C -1.703125 .083190 .15043$

$\begin{array}{llll}C & -0.71380 & 0.95067 & 0.42960\end{array}$

Rh $0.15086-0.56188-0.44692$

$\mathrm{Cl} 0.935740 .62029-2.56397$

C $-0.92410-0.08723 \quad 1.37136$

B $-2.21680-0.835390 .81520$

N $-2.10722-0.59391-0.59326$

C $-2.99331-0.73751-1.75910$

C $-3.09916-2.21255-2.22738$

C $-3.27526-1.548251 .82256$

C $-4.06315-2.74114 \quad 1.23052$

C $-2.49777-2.092143 .05298$

C $-4.27710-0.47847 \quad 2.33791$

C $-4.40084-0.19128-1.40361$

$\begin{array}{llll}\text { C } & -2.45258 & 0.11001 & -2.93232\end{array}$

$\begin{array}{lllll}\text { P } & 2.45948 & -0.28336 & 0.18867\end{array}$

C $2.71287 \quad 1.57010 \quad 0.60141$

$\begin{array}{llll}C & 4.12466 & 2.16120 & 0.43473\end{array}$

C $0.14048-2.72859-0.24044$

C $0.31245-2.48323-1.46743$

C $3.04439-1.37010 \quad 1.66369$

C $4.30537-0.901632 .42185$

C $3.74539-0.64123-1.19790$

C $5.21689-0.78525-0.76033$

C $1.92311-1.655762 .67990$

C $3.35457-1.85495-2.05816$

C $2.13123 \quad 1.947341 .97746$

H $\quad 3.27925-2.32099 \quad 1.14377$

H $3.631760 .25732-1.83090$

H $2.069062 .01286-0.18179$

H $-0.48439-0.077662 .37482$

H $\quad 0.44630-2.69620-2.51829$

H $\quad 0.00275-3.350960 .63330$

H -4.94946 -0.91164 3.10475

H -3.753110 .374782 .80660$

H $-4.90882-0.07587 \quad 1.52833$

H $-3.19063-2.585403 .76172$

H $-1.73867-2.841362 .76170$

H - $1.98037-1.289453 .60626$

H $-4.70910-3.196392 .00692$

H $-4.71929-2.44906 \quad 0.39524$

H -3.38576 -3.531320 .86057$

$\mathrm{H}-3.77766-2.28777-3.09641$

H $-2.11000-2.59003-2.52898$

H -3.48476 - $2.86538-1.42944$

H $-3.12640 \quad 0.01924-3.80273$

H $-2.39304 \quad 1.17397-2.64866$

H $-1.44052-0.20729-3.22361$

H $-5.05546-0.23077-2.29219$

H $-4.88464-0.77361-0.60478$

H $-4.330590 .85917-1.07431$

H 2.044453 .046092 .05161

H $1.12819 \quad 1.52795 \quad 2.14564$

H $2.78378 \quad 1.614962 .80221$

H $4.07252 \quad 3.253720 .59719$ 
H $4.839641 .75791 \quad 1.17149$

H $4.531932 .00397-0.57589$

H $2.24896-2.447203 .37942$

H $1.69636-0.759913 .28337$

H $0.99230-1.977242 .19238$

H $5.16801-0.706361 .77243$

H 4.103970 .011043 .00635

H $4.60355-1.684543 .14362$

H $4.14331-2.02880-2.81308$

H $3.25594-2.77988-1.46331$

H $2.41379-1.65925-2.58803$

H $5.84382-0.84789-1.66872$

H $5.59383 \quad 0.05665-0.16243$

H $5.38348-1.71872-0.19553$

H -2.044612 .398722 .25209$

$\mathrm{H}-2.594314 .838612 .11861$

H $-1.95346 \quad 6.147700 .08666$

H $-0.753615 .03206-1.80252$

H $-0.182732 .57746-1.65388$

TS(D2-E4)

SCF Energy $=-1269.04015227$

Enthalpy $0 \mathrm{~K}=-1268.376523$

Enthalpy $298 \mathrm{~K}=-1268.332078$

Free Energy 298K = -1268.451090

$\mathrm{SCF}(\mathrm{C} 6 \mathrm{H} 6)=-1269.04567293$

$\mathrm{SCF}(\mathrm{BP} 86-\mathrm{D} 3)=-0.14598125$

Lowest Frequencies $=-104.988621 .5226 \mathrm{~cm}-1$

C -1.10189-0.74382 -3.18127

C $-1.38688-1.88227-2.83581$

Rh $-0.30260-0.38761-0.43103$

B $0.099931 .50972-0.83152$

C $-0.020782 .79334-1.76088$

C $1.08062 \quad 2.57758-2.84517$

$\mathrm{Cl}-0.63787-2.88092 \quad 0.14671$

$\begin{array}{llll}1 \mathrm{P} & -2.34325 & -0.07908 & 0.67520\end{array}$

C $-3.70662-1.05283-0.25081$

C $-4.86220-1.645300 .57660$

C $1.00168 \quad 1.097640 .35747$

$\begin{array}{llll}\text { C } & 2.14696 & 0.22085 & 0.10870\end{array}$

N $1.84338-0.67343-0.81570$

C $2.74232-1.73663-1.36335$

C $1.99350-2.40404-2.53148$

C $-2.22069-0.87562 \quad 2.41397$

C $-3.33371-0.537873 .42419$

$\begin{array}{llll}\text { C } & -3.01623 & 1.70258 & 0.87258\end{array}$

$\begin{array}{llll}C & -4.47854 & 1.86986 & 1.33422\end{array}$

C $-0.83472-0.642843 .04613$

C -2.087172 .572041 .74069$

C $-4.21890-0.26745-1.47273$

C $4.03985-1.08916-1.90338$

C $3.06349-2.81232-0.29866$

C $-1.383702 .99548-2.46060$

C $0.333254 .08182-0.97039$

H $-2.947652 .06554-0.17028$

H -3.09849 $-1.90141-0.61425$

H $-2.27348-1.947202 .14575$

$\begin{array}{llll}\mathrm{H} & 0.94465 & 1.54783 & 1.35902\end{array}$

$\begin{array}{llll}\text { C } & 3.38910 & 0.38963 & 0.93529\end{array}$

H - $1.57231-2.85818-2.42223$

H $-0.881840 .21207-3.61522$

H $1.12722 \quad 3.46335-3.50765$ 
H $2.07908 \quad 2.44361-2.39380$

H $0.88197 \quad 1.69418-3.47570$

H $0.353094 .95688-1.64852$

H $-0.40906 \quad 4.29331-0.18129$

H $\quad 1.32240 \quad 4.00090-0.48875$

H - $1.326873 .82354-3.19311$

H $-1.719962 .09312-2.99714$

H $-2.17355 \quad 3.25732-1.73511$

H $4.65112-1.86373-2.39761$

H $3.80655-0.31106-2.65052$

H $4.64641-0.63936-1.10257$

H $3.58130-3.65926-0.78223$

H $3.72398-2.420550 .49000$

H $2.12861-3.18386 \quad 0.15183$

H $2.64104-3.16490-3.00019$

H $1.07817-2.89263-2.16491$

H $1.71009-1.66310-3.29634$

H - $0.76246-1.218363 .98743$

H $-0.03612-0.981202 .36874$

H $-0.66451 \quad 0.419683 .29334$

H $-3.22914-1.19897 \quad 4.30437$

H -3.248730 .498603 .79348$

H $-4.34936-0.678473 .02641$

H -2.39922 3.630831 .68201

H -2.13412 2.276952 .80266

H -1.03515 $2.51461 \quad 1.42287$

$\mathrm{H}-4.766542 .933331 .23800$

$\begin{array}{llll}\mathrm{H} & -5.18949 & 1.28373 & 0.73362\end{array}$

H $-4.61484 \quad 1.59092 \quad 2.39005$

H $-3.38853 \quad 0.13917-2.07051$

H $-4.79756-0.94252-2.12872$

H $-4.888020 .56252-1.18899$

H -5.49145 - 2.26750 - 0.08657

H $-4.50098-2.298711 .38627$

H $-5.51785-0.87427 \quad 1.01504$

C $\quad 4.27234 \quad 1.45627 \quad 0.65930$

C $5.384741 .69014 \quad 1.48176$

C 5.614120 .879302 .60529

C $4.72394-0.164812 .90387$

C $3.61666-0.409852 .07679$

H $4.088312 .09572-0.21050$

H $\quad 6.070382 .51096 \quad 1.24645$

H $6.47971 \quad 1.06493 \quad 3.24953$

H $4.88877-0.792563 .78579$

H $2.91747-1.216402 .31773$

Intermediate E1

SCF Energy $=-1269.09617654$

Enthalpy $0 \mathrm{~K}=-1268.428397$

Enthalpy $298 \mathrm{~K}=-1268.385740$

Free Energy 298K = -1268.500536

$\mathrm{SCF}(\mathrm{C} 6 \mathrm{H} 6)=-1269.10251195$

$\mathrm{SCF}(\mathrm{BP} 86-\mathrm{D} 3)=-0.14808907$

Lowest Frequencies $=19.990328 .2132 \mathrm{~cm}-1$

C $1.515694 .20810-0.58362$

C $1.251882 .90038-0.14631$

C $1.944541 .80737-0.71420$

C $2.89712 \quad 2.05125-1.72974$

C $3.154303 .36135-2.16265$

C $2.464924 .44321-1.59166$

C $\quad 1.65978 \quad 0.38447-0.34572$

C $0.67198-0.55919-0.87371$ 
$\mathrm{Rh}-0.53673 \quad 0.036850 .77986$

Cl $-0.89901 \quad 1.423102 .72433$

N $2.36338-0.434110 .45850$

C $3.40417-0.12465 \quad 1.48231$

C $3.36665-1.266792 .52022$

C $4.79265-0.067030 .79907$

C $3.12208 \quad 1.213292 .20069$

B $1.56293-1.79371-0.16429$

C $2.47404-2.74610-1.15735$

C $3.46485-3.64816-0.38388$

C $1.49378-3.67688-1.91710$

C $3.26180-1.93493-2.21127$

P $-2.58452-0.01107-0.16900$

C $-2.858301 .35433-1.48734$

C $-4.06716 \quad 1.13435-2.42004$

C $\quad 0.64633-2.534700 .92617$

C $-0.48495-1.87817 \quad 1.24641$

C $-3.07935-1.72668-0.84559$

C $-4.58214-1.97859-1.08012$

C -3.829260 .449941 .21486$

$\begin{array}{llll}\text { C } & -5.21943 & 0.96051 & 0.79014\end{array}$

C $-2.24073-2.12110-2.07354$

C $-3.92057-0.665572 .27305$

C $-1.601561 .66203-2.31895$

H $-2.75295-2.37066-0.00734$

$\mathrm{H}-3.28150 \quad 1.28778 \quad 1.68552$

$\mathrm{H}-3.061112 .23133-0.84102$

H $\quad 0.27970-0.42386-1.88825$

H $-1.33234-2.22301 \quad 1.86168$

H $\quad 0.84611-3.51131 \quad 1.40022$

H $3.79399-2.60399-2.91624$

H $2.59167-1.29424-2.81383$

H $4.02232-1.28096-1.74811$

H $2.04262-4.37091-2.58472$

H $\quad 0.88975-4.28807-1.22330$

H $\quad 0.79339-3.09956-2.54700$

H $3.97424-4.35867-1.06572$

H $4.25694-3.064240 .11815$

H $2.95459-4.249540 .39008$

H $4.15963-1.106343 .27012$

H $2.39420-1.290553 .03746$

H $3.52956-2.247622 .04749$

H $5.56640 \quad 0.13226 \quad 1.56028$

H $5.03849-1.01908 \quad 0.30225$

H $4.82997 \quad 0.74206 \quad 0.05103$

H $3.82823 \quad 1.318543 .04212$

H $3.259822 .07998 \quad 1.53727$

H $2.09637 \quad 1.237752 .60660$

H - $1.796222 .54135-2.95953$

H $-0.72811 \quad 1.88781-1.68989$

H -1.33864 $0.82135-2.98287$

H $-4.220162 .04495-3.02748$

H $-3.882520 .30503-3.12299$

H $-5.00608 \quad 0.93075-1.88567$

H $-2.47745-3.16202-2.35793$

H $-2.46021-1.48522-2.94864$

H $-1.16465-2.06967-1.85596$

H $-4.72048-3.04219-1.34745$

H $-5.19301-1.78642-0.18516$

H - $4.98693-1.37975-1.91033$

H $-4.38373-1.588631 .88285$

H $-2.92766-0.905332 .68383$ 
H $-4.54417-0.315463 .11468$

$\begin{array}{llll}\mathrm{H} & -5.76780 & 1.26695 & 1.69965\end{array}$

H $-5.16467 \quad 1.846190 .13732$

$\begin{array}{llll}\mathrm{H} & -5.83157 & 0.19537 & 0.28561\end{array}$

H $3.42572 \quad 1.20709-2.18442$

H $3.89622 \quad 3.53442-2.94933$

H $2.668135 .46480-1.92920$

H $0.978385 .04572-0.12660$

H 0.532092 .725860 .66322

Intermediate E2

SCF Energy $=-1269.10372360$

Enthalpy $0 \mathrm{~K}=-1268.434497$

Enthalpy $298 \mathrm{~K}=-1268.392204$

Free Energy $298 \mathrm{~K}=-1268.505542$

$\mathrm{SCF}(\mathrm{C} 6 \mathrm{H6})=-1269.10923017$

$\mathrm{SCF}(\mathrm{BP} 86-\mathrm{D} 3)=-0.14365081$

Lowest Frequencies $=20.500628 .3889 \mathrm{~cm}-1$

C $-0.135443 .75079-1.06424$

C $0.83375 \quad 2.87961-0.59644$

C $0.86121 \quad 1.49726-1.00917$

C $-0.159491 .07276-1.97013$

C $-1.136382 .01510-2.42997$

C $-1.15727 \quad 3.31855-1.96287$

C $1.70355 \quad 0.42811-0.51435$

C $2.26552-0.65958-1.45418$

Rh $-0.41294-0.08377-0.02748$

Cl $-0.06715-1.202412 .06785$

$\begin{array}{lllll}\mathrm{N} & 2.85750 & 0.40170 & 0.36614\end{array}$

C $3.14817 \quad 1.06241 \quad 1.68302$

C $3.58835-0.042002 .67319$

C $4.275602 .09855 \quad 1.47292$

C 1.917101 .746042 .31376

B $3.53464-0.57975-0.45243$

C $4.99306-1.20366-0.67722$

C $5.97790-1.317600 .50711$

C $4.82437-2.61819-1.29492$

C $5.64346-0.27969-1.75466$

$\begin{array}{llll}\mathrm{P}-2.82232 & -0.35527 & 0.09719\end{array}$

C $-3.76722-0.54584-1.55772$

C $-5.25098-0.95330-1.47653$

C $1.34419-1.84499-1.51442$

C $0.12059-1.75074-0.95900$

C $-3.44032-1.644581 .35601$

$\begin{array}{llll}\text { C } & -4.88095 & -1.53280 & 1.88746\end{array}$

$\begin{array}{llll}C & -3.35094 & 1.36914 & 0.74524\end{array}$

$\begin{array}{llll}\text { C } & -4.80857 & 1.82282 & 0.54120\end{array}$

$\begin{array}{llll}\text { C } & -3.12689 & -3.06871 & 0.85623\end{array}$

C $-2.89977 \quad 1.54443 \quad 2.21056$

C $-3.01309-1.47370-2.53044$

H $-2.73805-1.443402 .18778$

H -2.715752 .025060 .11529$

H $-3.704450 .48525-1.96016$

H $2.48612-0.26997-2.47074$

H - $0.60804-2.56932-0.92229$

H $1.66321-2.76809-2.02053$

H $6.61630-0.70098-2.07442$

H $5.01267-0.18431-2.65584$

H $5.83077 \quad 0.73621-1.36427$

H $5.81000-3.03782-1.57406$

H $4.35191-3.31853-0.58391$

H $4.20235-2.59554-2.20607$ 
H $\quad 6.94816-1.71518 \quad 0.15093$

H $\quad 6.18417-0.344540 .98463$

H $5.61017-2.01010 \quad 1.28382$

H 3.843470 .410263 .64735

H $2.76136-0.755672 .81976$

H $4.47068-0.587132 .31420$

H 4.538222 .580952 .43048

H $\quad 5.18568 \quad 1.62505 \quad 1.06818$

$\begin{array}{llll}\text { H } & 3.96281 & 2.88761 & 0.76678\end{array}$

H 2.182002 .039103 .34406

H 1.603842 .660221 .79200

H $1.06917 \quad 1.045392 .36964$

H -3.48996-1.44100 -3.52742

H - $1.95577-1.18724-2.63813$

H -3.04097 -2.52280 -2.18894

H $-5.70768-0.88861-2.48179$

H $-5.35799-1.99874-1.14157$

H -5.84036 - $0.31689-0.80013$

H $-3.34659-3.797361 .65755$

H $-3.73811-3.35026-0.01924$

H $-2.06104-3.17706 \quad 0.60027$

H -5.02326 -2.27344 2.69671

H -5.09915 -0.541502 .31495$

H $-5.63738-1.745101 .11552$

H $-3.53997 \quad 0.973172 .90452$

H $-1.86121 \quad 1.208972 .37242$

H -2.96852 2.608702 .49945

$\begin{array}{llll}\text { H } & -4.91908 & 2.86269 & 0.90141\end{array}$

H -5.10742 $1.81360-0.51959$

H $-5.52586 \quad 1.204431 .10530$

H $\quad 0.025920 .19165-2.59392$

H - $1.852131 .69010-3.19368$

H - $1.905164 .03168-2.32336$

H $-0.09370 \quad 4.80497-0.76778$

H $1.644493 .26098 \quad 0.02678$

Intermediate E3

SCF Energy $=-1269.11283950$

Enthalpy $0 \mathrm{~K}=-1268.443618$

Enthalpy $298 \mathrm{~K}=-1268.401456$

Free Energy $298 \mathrm{~K}=-1268.515882$

$\mathrm{SCF}(\mathrm{C} 6 \mathrm{H} 6)=-1269.11949056$

$\mathrm{SCF}(\mathrm{BP} 86-\mathrm{D} 3)=-0.14432822$

Lowest Frequencies $=14.107824 .3702 \mathrm{~cm}-1$

C $1.870022 .12306 \quad 0.00224$

C $1.666290 .68359-0.45732$

C $\quad 1.059020 .51790-1.83031$

C $-0.07298-0.21735-1.92400$

$\mathrm{Rh}-0.79577-1.15658-0.36949$

$\begin{array}{llll}\text { P } & -2.50161 & 0.24349 & 0.03609\end{array}$

C $-3.436510 .56962-1.60014$

C $-2.782691 .67764-2.44952$

B $2.09919-1.148490 .59107$

N $2.84916-0.24099-0.21801$

C $4.18437-0.19031-0.88730$

C $4.29961-1.39120-1.85660$

C $2.24805-2.60345 \quad 1.24750$

C $0.79313-0.204140 .53020$

Cl -2.26367 -2.83966 - 1.34351

C $4.375011 .11471-1.69060$

C $5.29383-0.253380 .18775$

C $-3.66965-0.79452 \quad 1.13348$ 
C $-2.85425-1.650642 .12530$

C $-2.12642 \quad 1.913010 .87947$

C $-1.66419 \quad 1.738842 .34069$

C $-4.80496-0.04384 \quad 1.85456$

$\begin{array}{llll}C & -3.25862 & 2.95931 & 0.81058\end{array}$

$\begin{array}{llll}C & -4.95978 & 0.77198 & -1.48691\end{array}$

$\begin{array}{llll}\mathrm{H} & -1.26545 & 2.27744 & 0.28802\end{array}$

H -3.26015 $-0.40425-2.09435$

$\begin{array}{llll}\text { H } & -4.09381 & -1.48507 & 0.37967\end{array}$

$\begin{array}{lllll}\mathrm{H} & 0.57142 & 0.32952 & 1.47023\end{array}$

H $-0.58813-0.43266-2.87299$

H $1.553670 .91748-2.72748$

H $5.378851 .10310-2.14806$

H $3.643691 .21118-2.50863$

H $4.302742 .00690-1.05035$

H $6.28918-0.25731-0.29028$

$\begin{array}{llll}\text { H } & 5.23679 & 0.62480 & 0.85252\end{array}$

H $5.20973-1.160290 .80377$

H $5.27839-1.38784-2.36829$

H $4.19935-2.34785-1.31858$

H $3.50597-1.34644-2.62101$

H $-3.53345-2.337832 .66206$

H -2.11397 -2.284801 .60366$

H $-2.33150-1.037182 .87697$

H -5.48065 -0.784042 .32096$

$\begin{array}{llll}\mathrm{H} & -4.42419 & 0.59907 & 2.66528\end{array}$

H $-5.41257 \quad 0.57744 \quad 1.18003$

H -1.21515 2.683292 .69453

H -2.51164 1.506243 .00686

H $-0.90917 \quad 0.94817 \quad 2.45723$

H $-2.89511 \quad 3.903601 .25508$

H $-3.580513 .18450-0.21646$

H $-4.143632 .64658 \quad 1.38757$

H -3.02409 $2.68680-2.07627$

H - $1.686831 .58058-2.49331$

H $-3.168391 .61348-3.48267$

H $-5.378610 .85328-2.50646$

H -5.46164 - $0.07862-0.99991$

H $-5.22915 \quad 1.69681-0.94981$

C $3.66776-3.13095 \quad 1.55144$

C $1.43620-2.621862 .57012$

C $1.56154-3.570210 .24309$

H $1.40002-3.644242 .99347$

H $1.88500-1.960273 .33301$

H $0.39551-2.287972 .40935$

H $3.61603-4.146231 .99060$

H $4.29458-3.203410 .64641$

H $4.19427-2.488832 .27970$

H $1.46152-4.583780 .67854$

H $\quad 0.52771-3.25019-0.02133$

H $2.12383-3.65741-0.70250$

C $2.603962 .39912 \quad 1.17833$

C $2.73473 \quad 3.709951 .65766$

C 2.131324 .779960 .97316

C $1.396634 .52111-0.19368$

C $1.26825 \quad 3.20590-0.67324$

$\begin{array}{lllll}\mathrm{H} & 3.08021 & 1.56907 & 1.71084\end{array}$

H 3.313143 .898912 .56886

$\begin{array}{lllll}\mathrm{H} & 2.23798 & 5.80455 & 1.34468\end{array}$

H $0.926735 .34564-0.74141$

H $0.699763 .01250-1.58943$ 
Intermediate E4

SCF Energy $=-1269.06039463$

Enthalpy $0 \mathrm{~K}=-1268.394173$

Enthalpy $298 \mathrm{~K}=-1268.350630$

Free Energy $298 \mathrm{~K}=-1268.466113$

$\mathrm{SCF}(\mathrm{C} 6 \mathrm{H} 6)=-1269.06555989$

$\mathrm{SCF}(\mathrm{BP} 86-\mathrm{D} 3)=-0.14979557$

Lowest Frequencies $=24.166028 .6911 \mathrm{~cm}-1$

C 1.002271 .601712 .04647

Rh 0.290960 .192560 .61538

B $-0.10002 \quad 1.65667-0.72984$

C $0.026643 .18762-1.12492$

C -1.11637 $3.86537-0.30376$

$\begin{array}{lllll}\text { C } & 0.88155 & 0.49302 & 2.65518\end{array}$

Cl $-0.05689-2.31478 \quad 1.13842$

P $2.39766-0.59028-0.14660$

C $3.39554-1.17710 \quad 1.38680$

C $4.34636-2.373461 .19893$

C $-0.986030 .49319-1.18805$

C $-2.103660 .13732-0.32226$

$\begin{array}{llll}\mathrm{N} & -1.89840 & 0.49618 & 0.93841\end{array}$

C -2.843790 .470492 .08715$

C -2.445461 .660162 .99555$

C $2.24534-2.14701-1.26000$

C $3.51040-2.54588-2.04724$

C $3.473550 .69085-1.07021$

C $4.976750 .38952-1.24454$

C $1.02815-2.10464-2.19981$

C $2.846991 .03297-2.43411$

C $4.11481-0.026242 .12126$

C $-4.309940 .68995 \quad 1.64100$

C $-2.72720-0.84508 \quad 2.88936$

C $\quad 1.359903 .89103-0.78487$

C $-0.281113 .38999-2.63346$

H $3.366611 .57183-0.40886$

H $2.55343-1.524882 .01366$

H $2.02358-2.91493-0.49708$

H $-0.94205 \quad 0.02072-2.17564$

C $-3.24702-0.64048-0.90069$

H $0.91946-0.18753 \quad 3.49617$

H 1.241202 .657342 .01396

H -1.16957 $4.93871-0.57011$

H $-2.100323 .41501-0.51893$

$\begin{array}{llll}\mathrm{H} & -0.94170 & 3.78999 & 0.78243\end{array}$

H $-0.322344 .46965-2.87487$

H $\quad 0.49530 \quad 2.93927-3.27638$

H - $-1.249352 .94005-2.91007$

H $1.290384 .97231-1.01096$

H $1.623623 .79185 \quad 0.28060$

H $2.197853 .48655-1.37617$

H $-4.92821 \quad 0.855532 .54030$

H $-4.40158 \quad 1.582910 .99881$

H -4.72417-0.17277 1.10051

H $-3.31878-0.76343 \quad 3.81873$

H $-3.10709-1.700612 .30983$

H -1.67888-1.06154 3.14401

H $-3.11171 \quad 1.70277 \quad 3.87454$

H -1.406591 .560003 .34090$

H $-2.53527 \quad 2.61306 \quad 2.44642$

H $0.95327-3.07314-2.72783$

H $0.10228-1.95646-1.62765$

H $1.11533-1.31646-2.96790$ 
C $1.61118-1.926622 .56874$

$\begin{array}{llll}C & 4.38826 & 1.39814 & 0.40708\end{array}$

C $4.07186-1.910662 .05993$

C $4.81872-1.50646-1.14289$

H $2.54312-2.887760 .87358$

H $3.34958-0.13955-1.98799$

H $2.31177 \quad 1.72484-0.01732$

$\begin{array}{llll}\mathrm{H} & -0.83270 & 0.26169 & 2.47026\end{array}$

H $-0.32763-2.59041-2.56100$

H $-0.28878-3.310420 .66465$

H $-4.77725 \quad-0.92742 \quad 3.36300$

H -3.651680 .423333 .05659$

H $-4.88677-0.000821 .84445$

H $-2.93009-2.529123 .79580$

H - $1.57779-2.751262 .66265$

H - $1.72860-1.238033 .59576$

H $-4.51037-3.160602 .08806$

H $-4.72455-2.250860 .57389$

H $-3.30338-3.298030 .77628$

$\mathrm{H}-3.54022-0.58711-3.78870$

H - $1.82836-0.72207-3.29529$

H -3.01509-1.98346-2.80136

H $-3.601371 .74956-2.77906$

H $-3.219801 .99837-1.04879$

H - $1.909531 .60585-2.21085$

H $-5.305720 .09735-2.12548$

H $-4.90633-1.39693-1.23779$

$\begin{array}{llll}\mathrm{H} & -4.93227 & 0.17949 & -0.38248\end{array}$

$\begin{array}{lllll}\mathrm{H} & 2.67122 & 2.46890 & 2.28246\end{array}$

H 1.458641 .171062 .31627

H 3.140220 .840082 .81813

H $4.558422 .45666 \quad 0.67825$

H $5.061910 .79137 \quad 1.03565$

H $4.690591 .28195-0.64506$

H $1.68406-2.857793 .15979$

H $1.78868-1.087313 .26119$

H $\quad 0.58278-1.829472 .19080$

H $4.88042-2.032891 .32877$

H $4.24822-0.971422 .60953$

H $4.15989-2.733562 .79363$

H $3.42021-2.37472-3.17974$

H $2.54796-3.11199-1.81319$

H $1.78431-1.79646-2.77179$

H $5.37413-1.52155-2.09883$

H $5.35615-0.83402-0.46074$

H $4.86681-2.53026-0.73355$

H -1.33343 3.004822 .34002

H - $1.00738 \quad 5.478892 .27394$

$\begin{array}{lllll}\mathrm{H} & 0.17810 & 6.53410 & 0.33761\end{array}$

H $1.021415 .10785-1.53839$

H 0.68687 2.61808-1.47191

TS(E1-F1)

SCF Energy $=-1269.09399787$

Enthalpy $0 \mathrm{~K}=-1268.426645$

Enthalpy $298 \mathrm{~K}=-1268.384696$

Free Energy 298K = -1268.496506

$\mathrm{SCF}(\mathrm{C} 6 \mathrm{H} 6)=-1269.09934885$

$\mathrm{SCF}(\mathrm{BP} 86-\mathrm{D} 3)=-0.14881738$

Lowest Frequencies $=-113.648828 .0214 \mathrm{~cm}-1$

C $1.27243 \quad 4.27583-0.26987$

C 1.135962 .927690 .09554 
C $\quad 1.73897 \quad 1.91249-0.68322$

C $2.473132 .27630-1.83362$

C $2.623913 .62697-2.18241$

C 2.02214 4.62937-1.40392

C $1.559210 .45650-0.38196$

C $0.85850-0.53027-1.15647$

Rh $-0.31585-0.07648 \quad 0.66012$ $\begin{array}{llll}\mathrm{Cl} & -0.84925 & 0.64390 & 2.90145\end{array}$

$\begin{array}{lllll}\text { N } & 2.04188 & -0.29639 & 0.66807\end{array}$

C $3.000470 .06282 \quad 1.75666$

C $2.91862-1.095622 .77376$

$\begin{array}{llll}\text { C } & 4.42329 & 0.14690 & 1.15223\end{array}$

C $2.68843 \quad 1.388542 .48469$

B $1.58134-1.84890-0.52295$

C $2.81544-2.53740-1.33434$

C $3.71734-3.41730-0.43884$

C $2.11838-3.47011-2.36778$

C $3.68862-1.53954-2.12641$

P $-2.51926-0.05212-0.14831$

C $-2.866501 .42432-1.32470$

C $-4.12491 \quad 1.29872-2.20798$

C $0.62538-2.746900 .35843$

C $-0.36235-2.038950 .92702$

C $-3.01810-1.69188-0.99220$

C $-4.52038-1.93093-1.24295$

$\begin{array}{llll}C & -3.74353 & 0.22359 & 1.29786\end{array}$

$\begin{array}{llll}\text { C } & -5.12677 & 0.82120 & 0.98061\end{array}$

C $-2.19116-1.95811-2.26244$

C $-3.85344-1.035142 .18045$

C $-1.657581 .79386-2.20170$

H $-2.68067-2.41876-0.22875$

$\begin{array}{llll}\mathrm{H} & -3.17335 & 0.96467 & 1.88918\end{array}$

H $-3.023672 .24630-0.59796$

H $0.44136-0.30290-2.14196$

H - $1.14166-2.35108 \quad 1.63809$

H $\quad 0.73714-3.818290 .59048$

H $4.47341-2.06783-2.70273$

H $3.08281-0.96591-2.85188$

H $4.19749-0.81457-1.46865$

H $2.87309-3.98902-2.99132$

H $1.50433-4.24449-1.87582$

H $1.45914-2.90436-3.05133$

H $4.45843-3.97534-1.04510$

H $4.28185-2.81853 \quad 0.29689$

H $3.12861-4.160620 .12731$

H $3.66521 \quad-0.948283 .57274$

H $1.91597-1.130323 .22994$

H $3.11210-2.064132 .28535$

H $5.146890 .37846 \quad 1.95292$

H $4.72339-0.803910 .68532$

$\begin{array}{lllll}\mathrm{H} & 4.48509 & 0.94788 & 0.39570\end{array}$

H $3.35537 \quad 1.470943 .36091$

H 2.872352 .265391 .84576

H $1.64698 \quad 1.402862 .84405$

H - $1.883482 .71696-2.76605$

H $-0.74911 \quad 1.97428-1.60991$

H - $1.436341 .00298-2.93832$

H $-4.312962 .26819-2.70474$

H $-3.974840 .55256-3.00608$

H $-5.03302 \quad 1.02901-1.65114$

H - $2.43174-2.96421-2.65087$

H - $2.41990-1.23516-3.06473$ 
$\begin{array}{cccc}\text { H } & -1.11305 & -1.92697 & -2.05062 \\ \text { H } & -4.66037 & -2.97655 & -1.57342 \\ \text { H } & -5.13527 & -1.78946 & -0.34154 \\ \text { H } & -4.92188 & -1.28322 & -2.03708 \\ \text { H } & -4.35867 & -1.87339 & 1.67080 \\ \text { H } & -2.86308 & -1.36492 & 2.52947 \\ \text { H } & -4.44502 & -0.79271 & 3.08134 \\ \text { H } & -5.65269 & 1.00778 & 1.93504 \\ \text { H } & -5.06262 & 1.78839 & 0.45683 \\ \text { H } & -5.76624 & 0.14782 & 0.38606 \\ \text { H } & 2.93091 & 1.49231 & -2.44535 \\ \text { H } & 3.20848 & 3.89553 & -3.06856 \\ \text { H } & 2.13443 & 5.68250 & -1.68174 \\ \text { H } & 0.79435 & 5.05075 & 0.33828 \\ \text { H } & 0.55727 & 2.65772 & 0.98737\end{array}$

TS(E1-F2)

SCF Energy $=-1269.07827857$

Enthalpy $0 \mathrm{~K}=-1268.410758$

Enthalpy $298 \mathrm{~K}=-1268.368793$

Free Energy 298K $=-1268.480744$

$\mathrm{SCF}(\mathrm{C} 6 \mathrm{H6})=-1269.08473650$

$\mathrm{SCF}(\mathrm{BP} 86-\mathrm{D} 3)=-0.15044276$

Lowest Frequencies $=-215.407023 .4279 \mathrm{~cm}-1$

C $2.84022 \quad 4.08447-0.09157$

C $2.094942 .89913-0.02086$

C $2.66151 \quad 1.65604-0.39062$

C $3.999901 .64274-0.84639$

C $4.741152 .83128-0.92764$

C $4.167464 .05519-0.54850$

C $1.793620 .44013-0.32312$

C $0.615930 .18563-1.05088$

Rh $-0.53906-0.174750 .58602$

$\mathrm{Cl}-1.01224 \quad 0.05164 \quad 2.96478$

$\begin{array}{llll}\text { N } & 1.95591 & -0.69620 & 0.48808\end{array}$

$\begin{array}{llll}\text { C } & 2.77415 & -0.83620 & 1.74734\end{array}$

C $2.22557-2.038042 .54353$

C $4.25434-1.10622 \quad 1.38121$

$\begin{array}{llll}\text { C } & 2.66996 & 0.43415 & 2.61469\end{array}$

B $1.35988-1.79649-0.45731$

C $2.17030-2.34660-1.77555$

C $2.81763-3.70307-1.36324$

C $1.17437-2.63523-2.92717$

C $3.29369-1.45044-2.32742$

$\begin{array}{llll}\text { P } & -2.63751 & 0.35847 & -0.15446\end{array}$

C $-2.84503 \quad 2.16309-0.78761$

C $-4.059992 .41466-1.70468$

$\begin{array}{llll}\text { C } & 0.24366 & -2.76386 & 0.11063\end{array}$

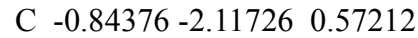

C $-3.22245-0.87362-1.49242$

C $-4.73038-0.92751-1.80615$

$\begin{array}{llll}\text { C } & -3.88848 & 0.28494 & 1.29673\end{array}$

$\begin{array}{llll}\text { C } & -5.21912 & 1.04491 & 1.13439\end{array}$

C $-2.38174-0.74296-2.77514$

C $-4.11835-1.157281 .78692$

C $-1.581652 .73884-1.44637$

H $-2.94038-1.83115-1.01503$

$\begin{array}{llll}\mathrm{H} & -3.29204 & 0.78837 & 2.08098\end{array}$

H -3.002242 .701760 .16786$

$\begin{array}{llll}\mathrm{H} & 0.38444 & 0.70807 & -1.98628\end{array}$

H - $1.79378-2.549510 .91436$

H $\quad 0.25200-3.864380 .05090$ 


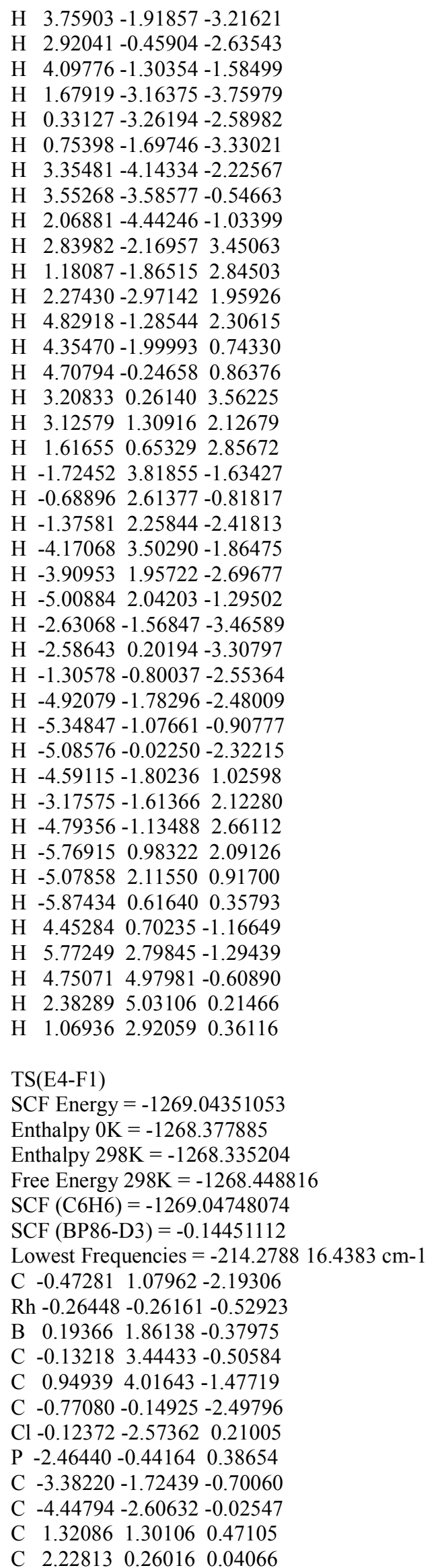


N $1.85027-0.43730-1.02770$

C $2.67334-1.38508-1.83286$

C $1.78594-1.92856-2.97115$

C $-2.37976-1.197012 .14758$

C $-3.67734-1.199362 .97971$

$\begin{array}{llll}\text { C } & -3.56644 & 1.11463 & 0.48530\end{array}$

$\begin{array}{llll}C & -5.08646 & 0.90104 & 0.63721\end{array}$

C $-1.21310-0.606842 .96488$

C $-3.052842 .06077 \quad 1.58702$

C $-3.92073-1.09412-2.00131$

C $3.83974-0.57836-2.45941$

C $3.23539-2.59245-1.03995$

C -1.51972 $3.88392-1.03079$

$\begin{array}{lllll}C & 0.10485 & 4.13477 & 0.86764\end{array}$

H $-3.378441 .58990-0.49648$

H - $2.52657-2.37474-0.95829$

H $-2.10464-2.238451 .90329$

H $1.56293 \quad 1.75494 \quad 1.44172$

C 3.477800 .075160 .86299

H - $1.04696-0.81495-3.31326$

H $-0.506662 .05639-2.66963$

H $0.857785 .11848-1.53144$

H $1.96843 \quad 3.77275-1.13266$

H $0.847083 .63064-2.50744$

$\begin{array}{llll}\mathrm{H} & -0.02600 & 5.22887 & 0.76568\end{array}$

H -0.602613 .789981 .64049$

H $1.126613 .95630 \quad 1.24089$

H - $1.549434 .98470-1.14148$

$\mathrm{H}-1.76545 \quad 3.45749-2.01881$

H $-2.329893 .60437-0.34078$

H $4.42296-1.23013-3.13346$

H $3.453240 .27225-3.04592$

H $4.52338-0.19267-1.68612$

H $3.71240-3.29528-1.74639$

H $3.99630-2.28633-0.30759$

H $2.42087-3.11643-0.51704$

H $2.37636-2.61514-3.60169$

H $0.92696-2.48185-2.55854$

H $1.41643-1.10780-3.60700$

H - $1.08947-1.193243 .89377$

H $-0.26582-0.653052 .40625$

H -1.396740 .440893 .25575$

H -3.51553 -1.81851 3.88149

H $-3.94725-0.190203 .33311$

H $-4.54263-1.618452 .44570$

H $-3.526543 .05427 \quad 1.48922$

H $-3.30402 \quad 1.680662 .59066$

H - 1.960882 .196451 .53972

H $-5.59442 \quad 1.882030 .58586$

$\begin{array}{llll}\mathrm{H} & -5.51535 & 0.26969 & -0.15431\end{array}$

H $-5.34413 \quad 0.45142 \quad 1.60835$

H $-3.20568-0.38897-2.45360$

H $-4.11970-1.89130-2.74023$

H $-4.87115-0.55754-1.84319$

H $-4.80381-3.35592-0.75643$

H $-4.04527-3.16116 \quad 0.83634$

H $-5.32971-2.03210 \quad 0.30569$

C $3.56108-0.974091 .80377$

C $4.67728-1.079492 .64786$

C $5.72304-0.145162 .56040$

C $\quad \begin{array}{llll}\text { C } & 54178 & 0.90666 & 1.63404\end{array}$

C 4.518641 .024670 .79946 
H $2.74460-1.701141 .86566$

H $4.72946-1.895513 .37657$

H $6.59550-0.233753 .21628$

H 6.450311 .642211 .56434

H $4.444211 .85607 \quad 0.09015$

Intermediate F1

SCF Energy $=-1269.12226088$

Enthalpy $0 \mathrm{~K}=-1268.453691$

Enthalpy $298 \mathrm{~K}=-1268.411076$

Free Energy $298 \mathrm{~K}=-1268.525620$

$\mathrm{SCF}(\mathrm{C} 6 \mathrm{H} 6)=-1269.12738899$

$\mathrm{SCF}(\mathrm{BP} 86-\mathrm{D} 3)=-0.14116089$

Lowest Frequencies $=19.264127 .0498 \mathrm{~cm}-1$

C $\quad 0.21300 \quad 1.19819-2.33251$

Rh $-0.46225-0.75313-0.30762$

B $0.80393 \quad 1.84223-1.05967$

C $1.13386 \quad 3.42119-0.96825$

C $2.215543 .73669-2.04047$

C $-0.465270 .04135-2.08659$

Cl $-1.60546-2.86266-0.86129$

$\begin{array}{llll}\text { P } & -2.54928 & 0.02866 & 0.41030\end{array}$

C $-3.90374-0.22901-0.90802$

C $-5.33051-0.52040-0.40857$

$\begin{array}{llll}\text { C } & 0.96139 & 0.81803 & 0.15491\end{array}$

$\begin{array}{llll}\text { C } & 2.02745 & -0.22729 & 0.05380\end{array}$

N $1.56971-1.30591-0.53424$

C $2.25968-2.54252-1.00454$

C $1.67277-2.84951-2.40238$

C $-2.95812-1.15519 \quad 1.84911$

C $-4.07548-0.756532 .82925$

C $-2.68104 \quad 1.833361 .01452$

$\begin{array}{llll}\text { C } & -4.09869 & 2.38440 & 1.27039\end{array}$

C $-1.65562-1.502962 .60189$

$\begin{array}{llll}\text { C } & -1.78118 & 2.09415 & 2.23898\end{array}$

C $-3.875120 .89273-1.96463$

C $3.79081-2.38735-1.13543$

C $1.91015-3.68822-0.02760$

$\begin{array}{llll}\text { C } & -0.13839 & 4.23489 & -1.33028\end{array}$

C 1.646623 .893190 .41053

H -2.248272 .379260 .15337$

H -3.51660 - $1.15441-1.37499$

H $-3.26120-2.060021 .28877$

$\begin{array}{llll}\mathrm{H} & 1.01557 & 1.30365 & 1.14573\end{array}$

C $3.34991-0.022590 .72648$

H - $1.02876-0.55603-2.82434$

H $\quad 0.20064 \quad 1.61972-3.35066$

H $2.434404 .82260-2.05923$

H $3.170193 .21870-1.83561$

H $1.890953 .44340-3.05362$

H $1.89165 \quad 4.97304 \quad 0.39433$

H $0.88860 \quad 3.74324 \quad 1.20039$

$\begin{array}{lllll}\mathrm{H} & 2.55656 & 3.35029 & 0.72121\end{array}$

H $\quad 0.08750 \quad 5.31921-1.35939$

H $-0.54253 \quad 3.94672-2.31569$

H $-0.940814 .08936-0.58406$

H $4.18304-3.27994-1.65189$

H $4.05400-1.50505-1.74285$

H $4.30185-2.30771-0.16503$

H $2.33410-4.63595-0.40182$

H $2.33421-3.49728 \quad 0.97342$

H $\quad 0.81692 \quad-3.802240 .05354$ 
H $\quad 2.10640-3.78877-2.78711$

H $\quad 0.57886-2.96107-2.34533$

H $1.91584-2.03883-3.10968$

$\mathrm{H}-1.85623-2.275543 .36709$

H $-0.89455-1.927861 .91802$

H $-1.22299-0.626823 .11205$

H $-4.27002-1.596803 .52143$

H -3.789710 .110223 .44890$

H $-5.02483-0.518502 .32557$

H $-1.70747 \quad 3.181562 .42168$

H -2.20204 1.639983 .15224

H $-0.76118 \quad 1.70520 \quad 2.10083$

H -4.029473 .458541 .52362$

$\begin{array}{llll}\mathrm{H} & -4.75846 & 2.29700 & 0.39473\end{array}$

H $-4.58863 \quad 1.880392 .11860$

H $-2.85148 \quad 1.11231-2.30834$

H $-4.46571 \quad 0.58194-2.84517$

H $-4.317721 .83247-1.59265$

H -5.97736 - $0.73535-1.27907$

H $-5.36482-1.405650 .24635$

$\begin{array}{llll}\mathrm{H} & -5.78115 & 0.32985 & 0.13052\end{array}$

C $3.69243-0.778161 .86980$

C $4.88396-0.516782 .56315$

C 5.752760 .493062 .11803

$\begin{array}{llll}\text { C } & 5.41779 & 1.25098 & 0.98373\end{array}$

$\begin{array}{llll}\text { C } & 4.21650 & 1.00611 & 0.30217\end{array}$

H $3.01454-1.564442 .21818$

H $5.13465-1.105463 .45182$

$\begin{array}{lllll}\mathrm{H} & 6.68541 & 0.69171 & 2.65594\end{array}$

H $6.090832 .03925 \quad 0.63083$

H $3.952291 .59963-0.57886$

Intermediate F2

SCF Energy $=-1269.09930835$

Enthalpy $0 \mathrm{~K}=-1268.429931$

Enthalpy $298 \mathrm{~K}=-1268.387437$

Free Energy 298K = -1268.502240

$\mathrm{SCF}(\mathrm{C} 6 \mathrm{H} 6)=-1269.10529679$

$\mathrm{SCF}(\mathrm{BP} 86-\mathrm{D} 3)=-0.14282189$

Lowest Frequencies $=13.808724 .3818 \mathrm{~cm}-1$

C $3.43398 \quad 4.32268-0.13041$

C $2.47718 \quad 3.34102 \quad 0.16316$

$\begin{array}{llll}\text { C } & 2.66819 & 1.99449 & -0.23305\end{array}$

C $3.861921 .66622-0.91756$

C $4.820262 .64751-1.20506$

C $4.61197 \quad 3.98149-0.81514$

C 1.601370 .988940 .00115

C $\quad 0.28279 \quad 1.23807-0.15625$

Rh $-0.72804-0.409260 .21185$

Cl -1.46119 -1.60245 2.20683

$\begin{array}{llll}\text { N } & 1.94199 & -0.40458 & 0.38899\end{array}$

C $2.51160-0.504941 .81100$

C $2.16261-1.888952 .39047$

C $4.03712-0.240291 .82790$

$\begin{array}{llll}\text { C } & 1.83997 & 0.54270 & 2.73061\end{array}$

B $2.04436-1.39171-0.71160$

C $3.03738-2.67473-1.01039$

C $3.96185-3.35490 \quad 0.03119$

C $2.19532-3.83643-1.61975$

C $3.99063-2.12587-2.12491$

$\begin{array}{llll}\text { P } & -2.88218 & 0.20362 & -0.17313\end{array}$

$\begin{array}{llll}\text { C } & -3.55115 & 1.20686 & 1.30643\end{array}$ 
C $-4.886461 .94286 \quad 1.08153$

C $1.03901-1.12988-1.89996$

C $-0.22572-0.68882-1.67598$

C $-3.23390 \quad 1.11027-1.81832$

C $-4.706091 .12217-2.27985$

C $-3.88287-1.42674-0.20406$

C $-5.38860-1.34750 \quad 0.11092$

C $-2.639602 .53097-1.85867$

C $-3.58584-2.23178-1.48352$

C $-2.487802 .16734 \quad 1.87278$

H $-2.666760 .49050-2.53849$

H -3.39418 -1.94590 0.64092

$\begin{array}{llll}\mathrm{H} & -3.69408 & 0.39567 & 2.04623\end{array}$

H $-0.089992 .19829-0.52462$

H $-0.94791-0.53865-2.49413$

H $1.27310-1.46189-2.92459$

H $4.55184-2.96560-2.57873$

H $3.45931-1.60651-2.93979$

H $4.73710-1.42616-1.70740$

H $2.86298-4.66573-1.92406$

H $1.48067-4.23831-0.87981$

H $1.61657-3.53311-2.50563$

H $4.65663-4.03299-0.50150$

$\begin{array}{lllll}\text { H } & 4.58057 & -2.65539 & 0.61294\end{array}$

H $3.40173-3.98653 \quad 0.74073$

H $2.59725-1.979443 .40113$

H $1.06993-2.003932 .47210$

H $2.55774-2.71076 \quad 1.78565$

H $4.43862-0.474102 .82927$

H $4.57489-0.858521 .09444$

H $4.263700 .81419 \quad 1.60885$

H $2.20915 \quad 0.38207 \quad 3.75805$

H $2.08340 \quad 1.577762 .44446$

H 0.745020 .416382 .73980

H -2.870472 .625062 .80285$

$\mathrm{H}-1.551341 .638922 .10733$

H -2.256352 .985191 .16919$

H -5.23779 2.347142 .04823

$\mathrm{H}-4.76742 \quad 2.80150 \quad 0.39931$

$\mathrm{H} \quad-5.68330 \quad 1.29621 \quad 0.68534$

H $-2.813802 .97083-2.85717$

H $-3.11348 \quad 3.19913-1.12035$

H - $1.554932 .53198-1.68359$

H $-4.75877 \quad 1.59559-3.27739$

H $-5.131980 .11257-2.37607$

H -5.35207 1.70651 -1.60675

H -4.02464 - $1.77453-2.38760$

H - $2.50154-2.34921-1.64558$

H -4.01990 -3.24260 - 1.38576

H $-5.79053-2.376530 .14643$

H $-5.58258-0.894541 .09609$

H $-5.96979-0.79745-0.64674$

H $4.027390 .63326-1.23807$

H $5.733692 .36935-1.74200$

H $5.36367 \quad 4.74587-1.03751$

$\begin{array}{llll}\mathrm{H} & 3.26502 & 5.35640 & 0.19048\end{array}$

H 1.572103 .606220 .72053

TS(F1-G)

SCF Energy $=-1269.09778122$

Enthalpy $0 \mathrm{~K}=-1268.429377$

Enthalpy $298 \mathrm{~K}=-1268.387295$ 
Free Energy 298K $=-1268.501182$

$\mathrm{SCF}(\mathrm{C} 6 \mathrm{H} 6)=-1269.10247043$

SCF $($ BP86-D3 $)=-0.14109175$

Lowest Frequencies $=-263.281919 .0916 \mathrm{~cm}-1$

C $\quad 0.36142 \quad 0.36810-2.17991$

Rh $-0.11173-0.62251-0.47879$

P $-2.30607-0.401980 .41126$

C $-3.56978-0.67594-0.98667$

C $-5.02666-0.99460-0.60511$

C $\quad 0.30480 \quad 1.74057-2.15244$

B $0.49223 \quad 2.32717-0.75900$

C $0.40923 \quad 3.90471-0.42341$

C $1.64508 \quad 4.54810-1.11749$

$\begin{array}{lllll}\text { C } & 0.90301 & 1.23984 & 0.30383\end{array}$

$\begin{array}{lllll}\text { C } & 1.86237 & 0.17188 & 0.07561\end{array}$

N $1.87423-0.41030-1.16796$

C $2.87752-1.37419-1.73716$

C $2.48210-1.65843-3.20298$

C $4.26022-0.68071-1.72571$

C $2.92311-2.71640-0.97284$

Cl $-0.75320-2.59127-1.68500$

C $-2.62639-1.69091 \quad 1.79611$

C $-3.89423-1.504992 .65602$

C $-2.61884 \quad 1.30416 \quad 1.21348$

C $-4.07718 \quad 1.78754 \quad 1.33483$

C $-2.54232-3.13469 \quad 1.26368$

C -1.888191 .404442 .56797$

C $-3.479670 .47584-2.00811$

C $-0.869774 .53606-1.02808$

$\begin{array}{lllll}C & 0.47052 & 4.24052 & 1.08281\end{array}$

$\begin{array}{llll}\mathrm{H} & -2.09661 & 1.96930 & 0.49983\end{array}$

H -3.12641 - $1.57088-1.46315$

H $-1.74802-1.520082 .44953$

H $\quad 0.85848 \quad 1.49523 \quad 1.37097$

C $2.76035-0.20644 \quad 1.22440$

H $0.32645-0.27823-3.06203$

H $0.170392 .27538-3.10500$

$\mathrm{H}-4.58300 \quad 1.85078 \quad 0.35934$

H $-4.68301 \quad 1.14293 \quad 1.99173$

H $-4.08588 \quad 2.802331 .77398$

H -1.843772 .459302 .89221$

$\begin{array}{llll}\mathrm{H} & -2.41257 & 0.84403 & 3.35964\end{array}$

H $-0.85478 \quad 1.027392 .51085$

H $-3.946201 .40373-1.63382$

H $-2.43518 \quad 0.70293-2.27615$

H $-4.01497 \quad 0.18890-2.93092$

H -5.61176-1.14546-1.53130

H -5.11081 - $1.92176-0.01675$

H -5.51273 - $0.18226-0.04105$

H -2.55666 -3.83861 2.11581

H $-3.40529-3.378730 .62048$

H $-1.63472-3.312400 .66756$

H -3.90135 -2.27441 3.45006

H $-3.94286-0.524603 .15318$

H $-4.81700-1.634452 .06954$

H $0.45635 \quad 5.33562 \quad 1.24663$

H $-0.38931 \quad 3.81713 \quad 1.63253$

H $1.38968 \quad 3.84929 \quad 1.55451$

H $1.654195 .64417-0.95352$

H $2.594204 .14698-0.71768$

H $1.641354 .37212-2.20718$

H - $0.874575 .63486-0.88546$ 
H $-0.95090 \quad 4.33831-2.11076$

H - $1.784974 .14007-0.55079$

H $3.58205-3.41744-1.51462$

H $3.32228-2.597760 .04502$

H $1.91408-3.15709-0.92303$

H $4.99064-1.32306-2.24679$

H $4.215640 .28726-2.25262$

H $4.62798-0.51201-0.70199$

H $3.22972-2.33807-3.64543$

H $1.49720-2.14936-3.25788$

H $2.46851-0.73312-3.80245$

C $2.45541-1.307062 .05195$

C $3.23713-1.583533 .18505$

C $4.33611-0.768213 .50018$

$\begin{array}{llll}\text { C } & 4.64148 & 0.33491 & 2.68590\end{array}$

C $3.85123 \quad 0.62290 \quad 1.56259$

H $1.59695-1.939441 .79779$

H $2.98646-2.439353 .82056$

H $4.94833-0.987224 .38107$

H $\begin{array}{llll}5.49058 & 0.98129 & 2.93147\end{array}$

H $4.07568 \quad 1.49645 \quad 0.94141$

Product $\mathrm{G}$

SCF Energy $=-1269.13630275$

Enthalpy $0 \mathrm{~K}=-1268.465067$

Enthalpy $298 \mathrm{~K}=-1268.422577$

Free Energy $298 \mathrm{~K}=-1268.537156$

SCF $($ C6H6) $=-1269.14146208$

SCF $($ BP86-D3) $=-0.1440502$

Lowest Frequencies $=19.572427 .4535 \mathrm{~cm}-1$

C $2.35796 \quad 1.70525-1.42266$

Rh $-0.129000 .01052-0.82424$

$\begin{array}{llll}\text { P } & -2.10171 & -0.64680 & 0.06559\end{array}$

C $-3.488380 .02944-1.06495$

C $-4.91161-0.54095-0.91880$

C $1.67004 \quad 2.76702-0.91514$

$\begin{array}{lllll}\text { B } & 0.72048 & 2.56453 & 0.28199\end{array}$

$\begin{array}{lllll}\text { C } & 0.00568 & 3.82924 & 0.98236\end{array}$

C $1.162264 .58530 \quad 1.70338$

$\begin{array}{lllll}\text { C } & 0.76326 & 1.11780 & 0.83618\end{array}$

$\begin{array}{lllll}\text { C } & 1.62831 & 0.08487 & 0.30258\end{array}$

N $2.290740 .37123-0.95203$

C $3.15520-0.63621-1.73529$

C $2.95332-0.39612-3.25558$

C $4.63711-0.42520-1.35595$

C $2.72829-2.09203-1.46069$

Cl $-0.76060-0.60891-3.02135$

C $-2.28650-2.555720 .14807$

C $-3.46794-3.09710 \quad 0.98098$

C $-2.41316-0.00714 \quad 1.84391$

C $-3.88073 \quad 0.13922 \quad 2.29460$

C -2.26195 -3.20297-1.24817

C $-1.58957-0.776132 .89614$

C $-3.46537 \quad 1.57031-1.04360$

C $-0.624914 .78395-0.06220$

C -1.067673 .484392 .03590$

H $-1.98763 \quad 1.00747 \quad 1.76683$

H $-3.09103-0.28335-2.04803$

$\begin{array}{llll}\mathrm{H} & -1.35142 & -2.83747 & 0.66937\end{array}$

$\begin{array}{llll}\mathrm{H} & 0.40436 & 0.88223 & 1.84616\end{array}$

C $2.21992-0.912961 .26458$

H $3.017861 .81203-2.28512$ 
H $1.862523 .72459-1.41421$

$\begin{array}{llll}\mathrm{H} & -4.45098 & 0.82427 & 1.64947\end{array}$

H $-4.41587-0.823092 .32942$

$\begin{array}{llll}\mathrm{H} & -3.89841 & 0.56173 & 3.31629\end{array}$

H $-1.59669-0.214123 .84774$

H - $2.01334-1.772373 .10685$

H $-0.53888-0.916562 .59910$

H $-3.829861 .98673-0.08796$

H -2.44480 $1.95089-1.22084$

H $-4.118301 .96128-1.84452$

H -5.56462 - $0.05408-1.66681$

H $-4.94660-1.62321-1.11952$

H $-5.35813-0.359510 .07108$

H -2.19051 -4.30063 - 1.13963

H -3.18736 -2.98740 - 1.80989

H - $-1.41805-2.84621-1.85728$

H $-3.41633-4.201420 .99268$

H $-3.45148-2.75952 \quad 2.02800$

H $-4.44253-2.821890 .54926$

$\mathrm{H}-1.40513 \quad 4.394412 .57013$

H -1.962263 .039051 .56549$

H -0.692622 .779242 .79962$

H $\quad 0.77584 \quad 5.500302 .19461$

H 1.633393 .962492 .48531

H 1.954094 .892230 .99813

H -1.026295 .693160 .42726$

H $0.108635 .11348-0.81771$

H $-1.459074 .30091-0.60167$

H $3.22021-2.73664-2.20767$

H $3.02849-2.44836-0.46767$

H $1.63640-2.20246-1.57483$

H $5.27158-1.08736-1.96948$

H $4.956900 .61433-1.54390$

H $4.81733-0.66338-0.29588$

H $3.43327-1.22602-3.79960$

H $1.88066-0.38721-3.51125$

H $3.422090 .53009-3.62451$

C $1.56943-2.115731 .60302$

C $2.09793-2.966842 .58617$

C $3.28665-2.625003 .25075$

C $3.93974-1.423412 .92934$

C $3.40800-0.571041 .94913$

H $\quad 0.65718-2.378551 .05801$

H $\quad 1.58150-3.901342 .83034$

H $3.70027-3.288304 .01740$

H $4.86149-1.142313 .44974$

H $3.90555 \quad 0.37737 \quad 1.71943$ 


\section{$\underline{\text { References }}$}

(1) Werner, H.; Wolf, J.; Schubert, U.; Ackermann, K. J. Organomet. Chem. 1983, 243, C63-C70.

(2) Paetzold, P.; von Plotho, C.; Schmid, G.; Boese, R.; Schrader, B.; Bougeard, D.; Pfeiffer, U.; Gleiter, R.; Schafer, W. Chem. Ber. 1984, 117, 1089-1102.

(3) Braunschweig, H.; Damme, A.; Jimenez-Halla, J. O. C.; Pfaffinger, B.; Radacki, K.; Wolf, J. Angew. Chem. Int. Ed. 2012, 51, 10034-10037.

(4) Doisneau, G.; Balavoine, G.; Fellebeen-Khan, T. J. Organomet. Chem. 1992, 425, 113 117.

(5) (a) Cheng, L.; Zhang, B.; Cheng, Y.; Xie, Z.; Wang, L.; Jing, X.; Wang, F. Adv. Funct. Mater 2010, 20, 3143-3153. (b) Zieschang, F.; Schreck, M. H.; Schmiedel, A.; Holzapfel, M.;

Klein, J. H.; Walter, C.; Engels, B.; Lambert, C. J. Phys. Chem. C, 2014, 118, 27698-27714.

(6) Sheldrick, G. M. Acta Crystallogr. A. 2008, A64, 112-122.

(7) Gaussian 09, Revision E.01, Frisch, M. J.; Trucks, G. W.; Schlegel, H. B.; Scuseria, G. E.; Robb, M. A.; Cheeseman, J. R.; Scalmani, G.; Barone, V.; Mennucci, B.; Petersson, G. A.; Nakatsuji, H.; Caricato, M.; Li, X.; Hratchian, H. P.; Izmaylov, A. F.; Bloino, J.; Zheng, G.; Sonnenberg, J. L.; Hada, M.; Ehara, M.; Toyota, K.; Fukuda, R.; Hasegawa, J.; Ishida, M.; Nakajima, T.; Honda, Y.; Kitao, O.; Nakai, H.; Vreven, T.; Montgomery, J. A. Jr.; Peralta, J. E, Ogliaro, F.; Bearpark, M.; Heyd, J. J.; Brothers, E.; Kudin, K. N.; Staroverov, V. N.; Kobayashi, R.; Normand, J.; Raghavachari, K.; Rendell, A.; Burant, J. C.; Iyengar, S. S.; Tomasi, J.; Cossi, M.; Rega, N.; Millam, J. M.; Klene, M.; Knox, J. E.; Cross, J. B.; Bakken, V.; Adamo, C.; Jaramillo, J.; Gomperts, R.; Stratmann, R. E.; Yazyev, O.; Austin, A. J.; Cammi, R.; Pomelli, C.; Ochterski, J. W.; Martin, R. L.; Morokuma, K.; Zakrzewski, V. G.; Voth, G. A.; Salvador, P.; Dannenberg, J. J.; Dapprich, S.; Daniels, A. D.; Farkas, Ö.; Foresman, J. B.; Ortiz, J. V.; Cioslowski, J.; Fox, D. J.; Gaussian, Inc., Wallingford CT, 2009. 